\title{
Heizung der Atmosphären Weißer Zwerge durch Zyklotronstrahlung am Beispiel des Polars AM Herculis
}

\author{
Dissertation \\ zur Erlangung des Doktorgrades \\ der Mathematisch-Naturwissenschaftlichen Fakultäten \\ der Georg-August-Universität zu Göttingen
}

vorgelegt von

Matthias König

aus Hofgeismar

Göttingen 2005 
D 7

Referent: Prof. Dr. Klaus Beuermann

Koreferent: Prof. Dr. Stefan Dreizler

Tag der mündlichen Prüfung: 28. April 2005 
"The Truth is Out There"

Fox Mulder 



\section{Inhaltsverzeichnis}

1 Einleitung 1

1.1 Polare . . . . . . . . . . . . . . . . . . . . . . 1

1.2 Das Akkretionsszenario . . . . . . . . . . . . . . . . . . 2

1.3 Beobachtungen von AM Her . . . . . . . . . . . . . . 10

1.4 Aufbau der Arbeit . . . . . . . . . . . . . . . . . . . . . . 14

2 Atmosphärenmodellierung mit Einstrahlung $\quad 17$

2.1 Atmosphärenmodell . . . . . . . . . . . . . . . . . . . . . . 17

2.1 .1 Grundlagen . . . . . . . . . . . . . . . . . . . . . 17

2.1 .2 Geometrie . . . . . . . . . . . . . . . . . . 18

2.1 .3 Strahlungstransport . . . . . . . . . . . . . . . . . . 18

2.1.4 Hydrostatisches Gleichgewicht . . . . . . . . . . . . . 25

2.1.5 Strahlungsgleichgewicht und Temperaturkorrektur . . . . . . . 25

2.1 .6 Iterative Lösungsstruktur $\ldots \ldots \ldots \ldots$. . . . . . . . . . 27

2.1.7 Weitere Details zur Modellberechnung . . . . . . . . . . . 27

2.2 Ergebnisse . . . . . . . . . . . . . . . . . . . 28

2.2 .1 Isotrope Bestrahlung mit Schwarzkörperstrahlung . . . . . . 28

2.2.2 Winkelabhängige Bestrahlung . . . . . . . . . . . . . 36

3 Dreidimensionale Modellierung eines bestrahlten Sterns $\quad 47$

3.1 Methode . . . . . . . . . . . . . . . . . . . 47

3.1 .1 Ziele und Voraussetzungen . . . . . . . . . . . . . . . . . . 47

3.1 .2 Systemgeometrie . . . . . . . . . . . . . . . . . . 48

3.1 .3 Weltobjekte . . . . . . . . . . . . . . . . . . . 49

3.1.4 Berechnung der physikalischen Größen . . . . . . . . . . 54

3.1 .5 Berechnung des Gesamtspektrums . . . . . . . . . . . . . . 57

3.2 Ergebnisse . . . . . . . . . . . . . . . . . . . . . . 59

3.2 .1 Punktquelle . . . . . . . . . . . . . . . . . . . . 59

3.2.2 Kreisförmige Emissionsregion . . . . . . . . . . . . 63

4 Anwendung auf Beobachtungen von AM Her 73

4.1 Modellierung . . . . . . . . . . . . . . . . . 73

4.1.1 Unabhängige Modellierung der Phase $\varphi_{\text {orb }}=0.1 \ldots$. . . . 74 
4.1.2 Konsistente Modellierung mehrerer Phasen . . . . . . . . . . . 76

4.2 Diskussion der Ergebnisse . . . . . . . . . . . . . . . 80

4.2 .1 Das Atmosphärenmodellgitter . . . . . . . . . . . . . . 80

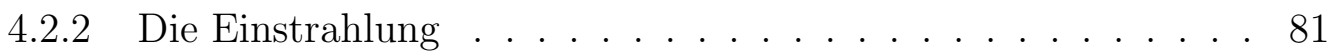

4.2 .3 Akkretionsregion . . . . . . . . . . . . . . . . . . . . . 82

4.2 .4 Systemgeometrie . . . . . . . . . . . . . . . 82

5 Zusammenfassung und Ausblick $\quad 83$

5.1 Zusammenfassung . . . . . . . . . . . . . . . . . . . 83

5.2 Ausblick . . . . . . . . . . . . . . . . . . . . . . . 84

\begin{tabular}{lr}
\hline A wd_model Referenz & $\mathbf{8 7}$
\end{tabular}

A.1 Organisation der Daten . . . . . . . . . . . . . . . . . . . . . . 87

A.2 wd_model.in Format . . . . . . . . . . . . . . . . . . . 87

A.3 wd_model Parameter . . . . . . . . . . . . . . . . . . . . . . . . . 88

A.4 wd_model Ausgabe . . . . . . . . . . . . . . . . . . . . . . . 91

A.4.1 Informationen über Flächenelemente . . . . . . . . . . . . . . 91

A.4.2 Spektrale Daten . . . . . . . . . . . . . . . . . 92

\begin{tabular}{ll}
\hline Danksagung & 97
\end{tabular}

\begin{tabular}{ll}
\hline Lebenslauf & 99
\end{tabular} 


\section{Kapitel 1}

\section{Einleitung}

\section{$1.1 \quad$ Polare}

Polare (AM Her Systeme) sind eine spezielle Klasse der Kataklysmischen Veränderlichen $(\mathrm{CVs})$, die wiederum in die Kategorie der engen Doppelsternsysteme („close binaries") fallen. Darunter versteht man Systeme bestehend aus zwei Sternen mit den Massen $M_{1}$ (Primärstern) und $M_{2}$ (Sekundärstern), in denen der Abstand der beiden Sterne von der gleichen Größenordnung ist wie der Durchmesser der größeren Komponente.

Begreift man das System idealisiert durch zwei Punktmassen, so ist die Orbitalbahn im Allgemeinen eine Ellipse. Der Zusammenhang zwischen der Orbitalperiode $P$, der großen Halbachse $a$ der Ellipse und den Massen $M_{1}, M_{2}$ ist gegeben durch das verallgemeinerte 3. Keplersche Gesetz

$$
P^{2}=\frac{4 \pi^{2} a^{3}}{G\left(M_{1}+M_{2}\right)}
$$

wobei $G=6.6726 \cdot 10^{-8} \mathrm{~cm}^{3} \mathrm{~g}^{-1} \mathrm{~s}^{-2}$ die Gravitationskonstante ist.

Der geringe Abstand der beiden Komponenten des Systems führt durch die gravitativen Gezeiteneffekte zu Verzerrungen im Sekundärstern. Im allgemeinen geht man jedoch davon aus, dass dies keine großen Effekte auf das Gravitationsfeld hat. Wenn der Großteil der Masse im Kern des Sterns sitzt, ist dies gerechtfertigt.

Eine anschauliche Beschreibung der im System wirkenden statischen Kräfte liefert das Roche-Potential: Neben den gravitativen Kräften durch die Punktmassen $M_{1}$ und $M_{2}$ liefert die Transformation auf ein mitrotierendes Bezugssystem die Zentrifugalkraft und die Corioliskraft. Beschränkt man sich auf die statischen Kräfte, so können diese durch ein Potential $\varphi(\vec{r})$ dargestellt werden. In dieser Darstellung ist ein mitrotierendes kartesisches Koordinatensystem $(x, y, z)$ gewählt. Der Ursprung $O=(0,0,0)$ wird identifiziert mit dem Massenzentrum des Primärsterns, der Punkt $(a, 0,0)$ mit dem des Sekundärsterns. Das Zentrum der Rotation liegt dann im Schwerpunkt $S=a M_{2} /\left(M_{1}+M_{2}\right)(1,0,0)$ des Systems. Die Rotationsachse ist parallel zur z-Achse, so dass die Rotation in der $x-y$-Ebene stattfindet. Das 
Roche-Potential $\varphi(\vec{r})$ ist durch den Ausdruck

$$
\begin{aligned}
\varphi(\vec{r})=-\frac{G M_{1}}{\sqrt{x^{2}+y^{2}+z^{2}}}-\frac{G M_{2}}{\sqrt{(x-a)^{2}+y^{2}+z^{2}}} \\
-\frac{1}{2} \omega_{\text {orb }}^{2}\left[\left(x-\frac{a M_{2}}{M_{1}+M_{2}}\right)^{2}+y^{2}\right]
\end{aligned}
$$

gegeben, wobei $\vec{r}=(x, y, z)$ den Positionsvektor und $\omega_{\text {orb }}=2 \pi / P$ die Orbitalkreisfrequenz bezeichnet. In Abbildung 1.1 sieht man verschiedene Darstellungen des Roche-Potentials. Wichtig für das Verständnis von Akkretion in einem CV-System ist der innere Langrangepunkt $L_{1}$. Er ist definiert durch den Sattelpunkt des Potentials. Die durch die Äquipotentialfläche, die den $L_{1}$-Punkt enthält, definierte geschlossene Fläche um eine Masse $M$ bezeichnet man als Roche-Fläche der Masse, bzw. das eingeschlossene Volumen als ihr Roche-Volumen.

In getrennten Systemen („detached systems“), in denen beide Komponenten innerhalb ihrer Roche-Volumina liegen, findet keine Akkretion statt. In halbgetrennten Systemen („semi-detached systems“), bei denen der Sekundärstern sein RocheVolumen überschreitet, der kompakte Primärstern jedoch innerhalb seines Volumens liegt, findet ein Massentransfer vom Sekundär- zum Primärstern statt. Bei Kontaktsystemen reichen beide Komponenten bis an die Grenzfläche heran. Kataklysmische Veränderliche sind halbgetrennte Systeme, bei denen der Primärstern ein Weißer Zwerg und der Sekundärstern ein Hauptreihenstern späten Spektraltyps ist. Einen Überblick über die Klassifikation von CVs liefert Warner (1995).

Der Schwerpunkt dieser Arbeit liegt bei den Polaren oder auch AM Her-Systeme (benannt nach dem Prototypstern AM Herculis). Bei Polaren besitzt der Weiße Zwerg ein starkes Magnetfeld ( $B \approx 10-200 \mathrm{MG}$ ). Weiterhin ist die Orbitalperiode $P$ des Systems mit der Eigenrotationsperiode $P_{\mathrm{wd}}$ des Weißen Zwerges synchronisiert: $P=P_{\mathrm{wd}}$.

\subsection{Das Akkretionsszenario}

Die Materie des Sekundärsterns, der sein gesamtes Roche-Volumen ausfüllt, verlässt am $L_{1}$ Punkt die Atmosphäre und strömt in das Roche-Volumen des Primärsterns. Aufgrund der Corioliskraft im mitrotierenden Bezugssystem um den Weißen Zwerg erfährt der Strom eine Ablenkung senkrecht zur radialen Richtung. Der Strom bewegt sich auf einer Umlaufbahn und bildet aufgrund der Viskosität der Materie eine Akkretionsscheibe aus. Besitzt der Primärstern ein Magnetfeld wird die Bewegung des ionisierten Materiestroms ab einer gewissen Entfernung vom Magnetfeld dominiert - die Region in der dieses geschieht definiert die Magnetosphäre. Dies führt zum Aufbrechen der Scheibe. Bei den Polaren ist das Magnetfeld so stark ( $B>10 \mathrm{MG}$ ), dass die Ausbildung einer Akkretionsscheibe vollständig verhindert wird. 

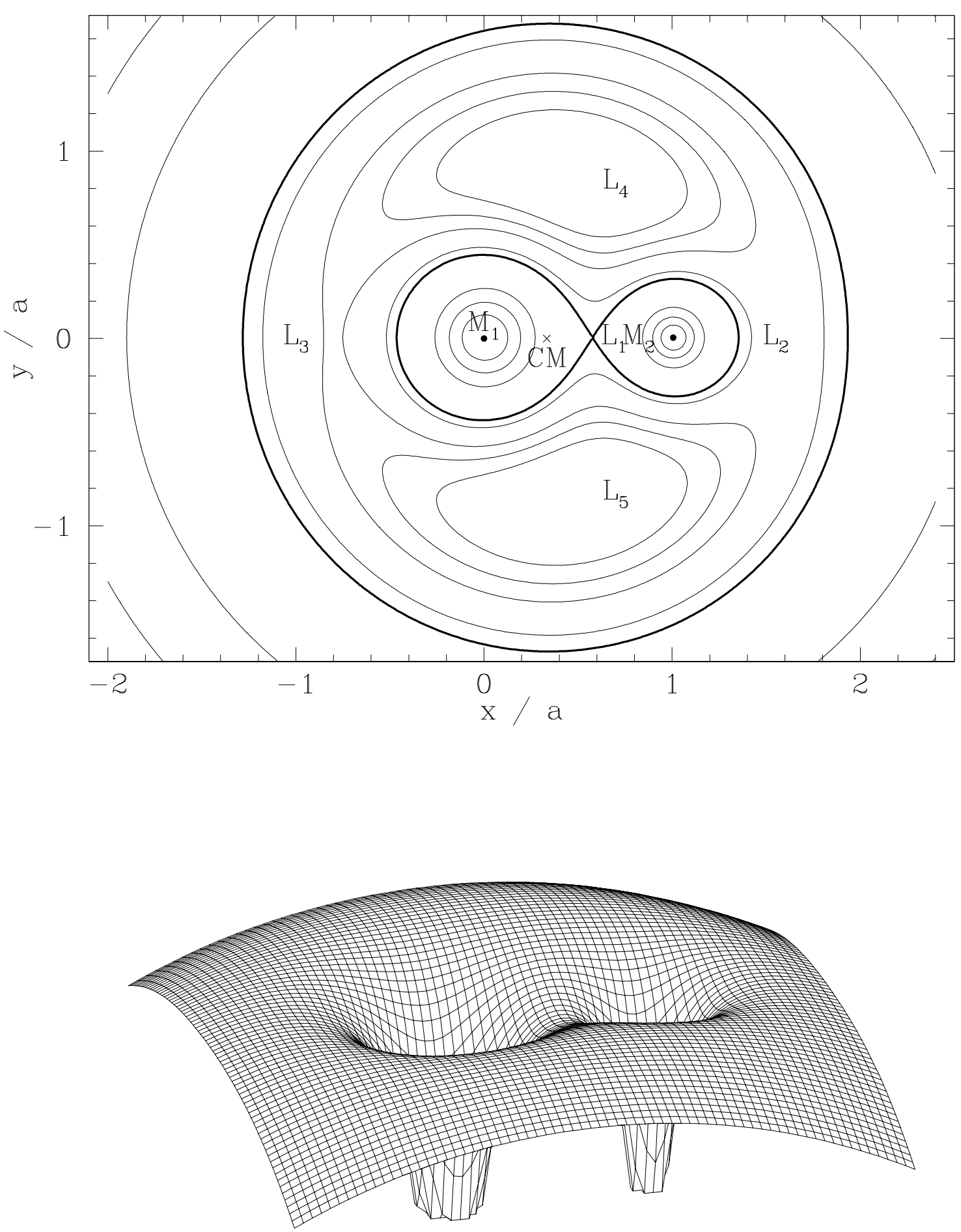

Abbildung 1.1: Oben: Äquipotentiallinien des Roche-Potentials $\varphi(\vec{r})$ im Schnitt durch die Ebene $z=0$ für zwei Punktmassen $M_{1}=0.6 M_{\odot}$ und $M_{2}=0.3 M_{\odot}$ mit einer Periode $P=3.5 \mathrm{~h}$. Die Koordinatenachsen haben die Einheit des Abstandes $a$. Die Rotationsachse läuft senkrecht durch den Schwerpunkt $C M$. Die Potentialniveaus der Linien sind so gewählt, dass die Struktur des Potential deutlich wird, sie repräsentieren keine äquidistanten Potentialwerte. Unten: Darstellung der Potentialfläche $\varphi(\vec{r})$. 
Der Radius der Magnetosphäre wird bestimmt durch das Gleichgewicht von magnetischem Druck $p_{\text {mag }}$ und dem Staudruck $p_{\text {ram }}$

$$
\frac{B^{2}}{8 \pi}=\rho v^{2}=\frac{\dot{M}}{\pi r_{\mathrm{st}}^{2}} v .
$$

Dabei ist $B$ die magnetische Induktion, $\rho$ die Dichte und $v$ die Geschwindigkeit des Materiestroms. Bei der letzten Umformung wurde ein zylinderförmiger Strom mit dem Radius $r_{\text {st }}$ angenommen, wobei der Zusammenhang zwischen der Dichte $\rho$ und dem Massenstrom $\dot{M}$ durch $\dot{M}=\pi r_{\text {st }}^{2} \rho v$ gegeben ist. Wenn das Magnetfeld $B$ groß genug ist, dass der Akkretionsstrom durch das Magnetfeld dominiert wird, fließt der Strom kanalisiert entlang der Magnetfeldlinien und trifft in einer Umgebung des magnetischen Pols bzw. der Pole auf die Oberfläche des Weißen Zwerges. In der Nähe der Oberfläche des Weißen Zwerges wird der Materiestrom ungefähr die Fluchtgeschwindigkeit $v_{\mathrm{ff}}=\sqrt{2 G M / R}$ besitzen, wobei $G$ die Gravitationskonstante, $M$ die Masse und $R$ der Radius des Weißen Zwerges sind.

Es bildet sich eine stehende Stoßwelle in der Höhe $x_{\mathrm{s}}$ über der Oberfläche aus, d.h. eine Unstetigkeit in den Materieparametern $v, \rho$ und T. Die Größen stehen in Beziehung durch die Rankine-Hugoniotschen Sprungbedingungen. Im Standardmodell wird eine starke Stoßwelle angenommen. Damit lauten die Relationen (Hoshi, 1973):

$$
\begin{aligned}
\rho_{2} & =\frac{\gamma+1}{\gamma-1} \rho_{1} \\
v_{2} \rho_{2} & =v_{1} \rho_{1} \equiv v_{\mathrm{ff}} \rho_{1} .
\end{aligned}
$$

Die Indizes 1, 2 beziehen sich auf die Größen vor bzw. nach dem Stoß. Dabei ist $\gamma$ der Adiabatenexponent. Nimmt man für die einfallende Materie ein einatomiges Gas an, ist $\gamma=5 / 3$. Dabei wird die Materie um den Faktor 4 abgebremst, entsprechend vergrößert sich die Dichte um den Faktor 4. Aus der Bedingung der Erhaltung des Impulsstroms

$$
p_{1}+\rho_{1} v_{1}^{2}=p_{2}+\rho_{2} v_{2}^{2}
$$

lässt sich ein Ausdruck für die Temperatur $T_{\mathrm{s}}$ an der Stelle des Stoßes herleiten. Aus der Annahme $p_{1}=0$ folgt zusammen mit (1.5)

$$
p_{2}=\frac{3}{4} \rho_{1} v_{\mathrm{ff}}^{2} \text {. }
$$

Mit der idealen Gasgleichung ergibt sich

$$
p_{2}=n_{2} k_{\mathrm{B}} T_{\mathrm{s}}=\frac{3}{4} \rho_{1} v_{\mathrm{ff}}^{2}
$$

und mit der Relation

$$
n_{2}=4 n_{1} \approx 4 \frac{\rho_{1}}{\mu m_{\mathrm{H}}}
$$




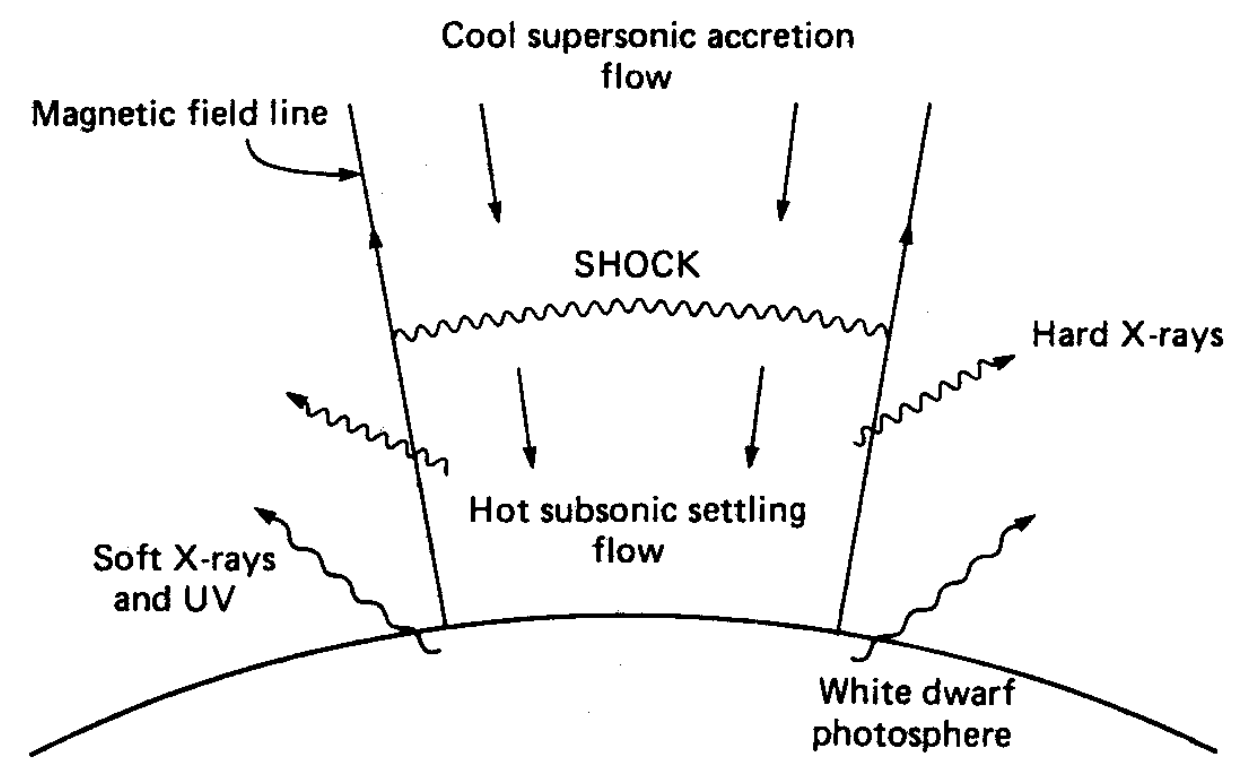

Abbildung 1.2: Schematische Darstellung der Akkretionsregion aus Frank et al. (1992). Der Akkretionsstrom läuft entlang der magnetischen Feldlinien mit Überschallgeschwindigkeit. Über der Oberfläche des Weißen Zwerges bildet sich eine Stoßwelle aus. Die Materie wird abgebremst und erhitzt. Dabei wird Strahlung freigesetzt, die auf die Atmosphäre des Weißen Zwerges trifft.

(mit dem mittleren Molekülgewicht $\mu$ ) folgt letztlich der von $\rho_{1}$ unabhängige Ausdruck

$$
k_{\mathrm{B}} T_{\mathrm{s}} \approx \frac{3}{8} \frac{\mu m_{\mathrm{H}} G M}{R+x_{\mathrm{s}}}
$$

Die Temperatur liegt in der Größenordnung $T_{s} \approx 10^{8} \mathrm{~K}$.

In der Region zwischen dem Stoß und der Oberfläche des Weißen Zwerges kühlt die Akkretionssäule durch verschiedene Mechanismen und gleicht sich den Bedingungen der Atmosphäre des Weißen Zwerges an. In den ursprünglichen Akkretionstheorien (Hoshi, 1973; Aizu, 1973) wurden ausschließlich Bremsstrahlungsverluste in Betracht gezogen. Später wurde deutlich, dass bei magnetisch akkretierenden Weißen Zwergen Zyklotron-Strahlung eine bedeutende Rolle spielen kann (Fabian et al., 1976; Masters et al., 1977; King \& Lasota, 1979 ; Lamb \& Masters, 1979).

Die genaue Struktur der Akkretionssäule in radialer Richtung hängt sehr stark davon ab, welcher Kühlungsmechanismus dominiert. Einen aktuellen Überblick, in dem verschiedene eindimensionale hydrodynamische Modelle der Akkretion gegenübergestellt werden, liefern Wu (2000), Fischer (2000) und Beuermann (2004).

Bei schwachen Magnetfeldern dominieren ausschließlich Bremsstrahlungsverluste. Das Plasma ist in diesem Bereich optisch dünn, so dass die Strahlungsverluste 
einfach aus der Bremsstrahlungsemissivität $\epsilon_{\mathrm{ff}}$ berechnet werden können, da in diesem Fall die Wechselwirkung zwischen Strahlung und Materie vernachlässigt werden kann.

Bei stärkeren Magnetfeldern sind die Verluste durch Zyklotronstrahlung nicht mehr zu vernachlässigen. Zyklotronstrahlung entsteht durch die Kreisbewegung der Elektronen im Magnetfeld (da das Verhältnis der Elektronen- zur Ionenbeschleunigung in der Größenordnung $\approx 8 \cdot 10^{4}$ liegt, kann die Abstrahlung der Ionen vernachlässigt werden). Im allgemeinen Fall, der relativistische Geschwindigkeiten einschließt, spricht man von Synchrotronstrahlung - den nicht-relativistischen Grenzfall nennt man Zyklotronstrahlung. Das Spektrum der Zyklotronstrahlung besteht aus einer Reihe von Harmonischen $\omega_{n}=n \omega_{\mathrm{c}}(n \in \mathbb{N} \backslash\{0\})$ der Zyklotrongrundfrequenz

$$
\omega_{\mathrm{c}}=2 \pi \nu_{\mathrm{c}}=\frac{e B}{m c} .
$$

Sofern die Harmonischen zu identifizieren sind, bieten sie eine Möglichkeit, das Magnetfeld am Ort der Emissionsregion zu bestimmen. In einem Plasma sind die Linien thermisch verbreitert und verschmelzen ab $n \approx \sqrt{m_{e} c^{2} / k_{\mathrm{B}} T}$ ineinander. Der Zyklotronabsorptionskoeffizient nimmt mit zunehmender Frequenz stark ab, so dass das Plasma bis zu einer Grenzfrequenz $\nu^{*}=m^{*} \omega_{\mathrm{c}}$ optisch dick ist und oberhalb von $\nu^{*}$ optisch dünn wird. Die Zyklotronstrahlung lässt sich folgendermaßen approximieren approximieren: Unterhalb der Grenzfrequenz $\nu^{*}$ strahlt das emittierende Plasma wie ein Schwarzkörper. Da $h \nu^{*} \ll k T$ ist, ist hier die Rayleigh-Jeans-Approximation zulässig. Die Grenzharmonische $m^{*}$ hängt ab von $\Lambda$ und $T$, wobei $\Lambda$ definiert ist durch

$$
\Lambda=\frac{\omega_{\mathrm{P}}^{2} l}{\omega_{\mathrm{c}} c}
$$

Dabei bezeichnet $\omega_{\mathrm{P}}=\sqrt{\left(4 \pi n_{\mathrm{e}} e^{2}\right) / m_{\mathrm{e}}}$ die Plasmafrequenz $\left(\omega_{\mathrm{P}}^{-1}\right.$ ist die Zeit, die ein Elektron im Mittel benötigt, um die Debeye-Länge $\lambda_{\mathrm{D}}=\sqrt{\left(k_{\mathrm{B}} T\right) /\left(4 \pi n_{\mathrm{e}} e^{2}\right)}$ zurückzulegen) und $l$ eine charakteristische Dimension des Plasmas (wie etwa die Dicke einer Plasmaschicht). Für die Akkretionsregion bei AM Her-Sternen liegt sie im Bereich $\Lambda \approx 10^{7}$ (Chanmugam \& Wagner, 1979). Die Grenzharmonische $m^{*}$ ist in Chanmugam \& Langer (1991) so definiert, dass der integrierte Modellzyklotronfluss $F_{\text {cyc }}$ dem bei $m^{*}$ abgeschnittenen integrierten Rayleigh-Jeans-Fluss entspricht:

$$
m^{*} \nu_{c}=\left(\frac{3 c^{2} F_{\mathrm{cyc}}}{2 \pi k T}\right)^{1 / 3}
$$

Diese wurde hier aus der von Chanmugam \& Langer (1991) angegebenen parametrisierten Formel

$$
\begin{aligned}
m^{*}(k T, \Lambda)= & {[4.54+0.409 k T]\left(\Lambda 10^{-7}\right)^{0.0786+9.58 \cdot 10^{-4} k T} } \\
& \cdot\left(1.02-2.17 \cdot 10^{-3}\left[\ln \left(\Lambda 10^{-6}\right)\right]^{2}\right)
\end{aligned}
$$




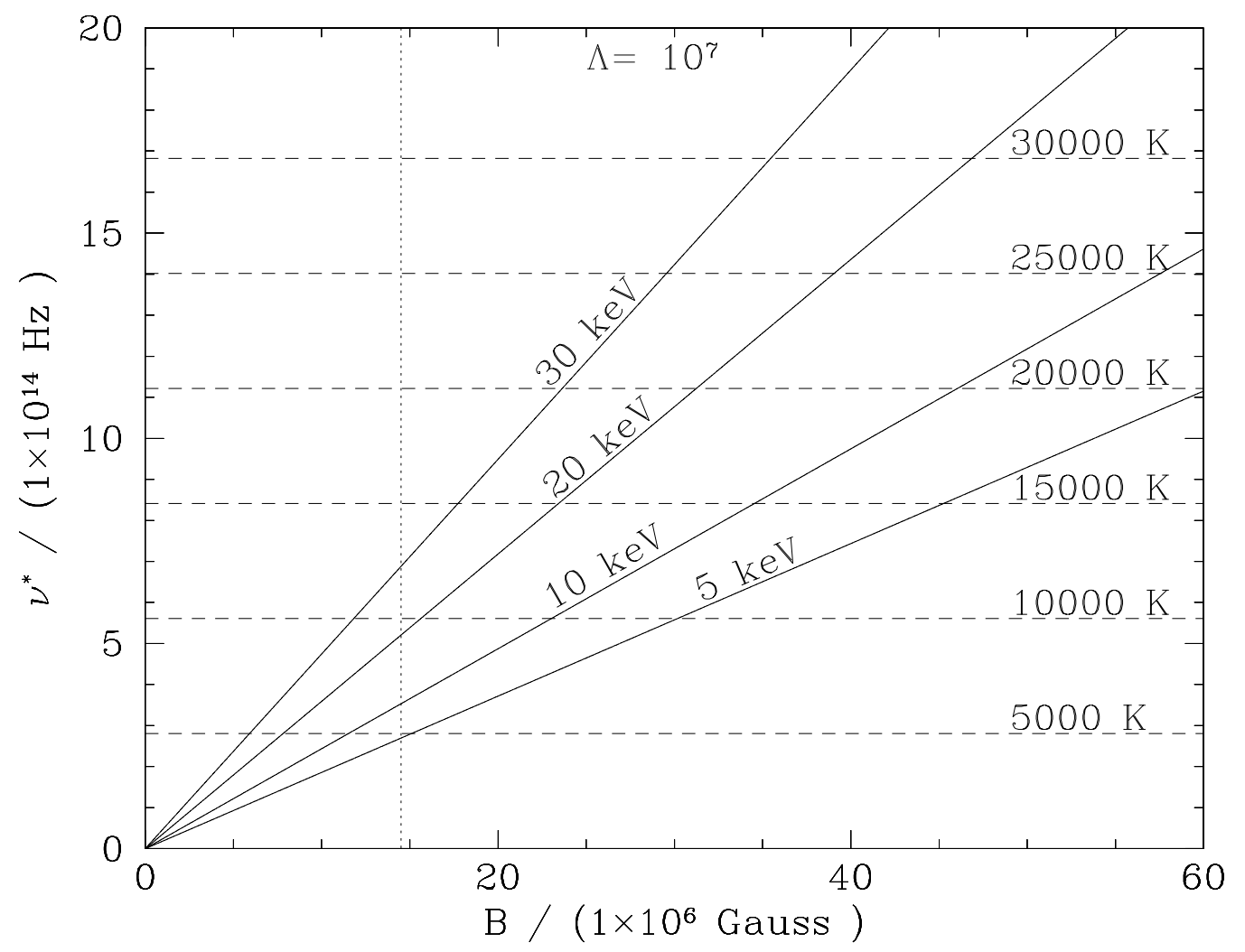

Abbildung 1.3: Die Rayleigh-Jeans-Abbruchfrequenz $\nu^{*}$ in Abhängigkeit von der magnetischen Induktion $B$. Der dimensionslose Plasmaparameter beträgt $\Lambda=10^{7}$. Die einzelnen Kurven entsprechen den angegebenen Plasmatemperaturen. Die gepunktete Linie markiert die magnetische Induktion in der Akkretionsregion von AM Her mit $B=14.5 \mathrm{MG}$ (Bailey et al., 1991). Die gestrichelten Linien parallel zur Abszisse entsprechen denjenigen Schwarzkörpertemperaturen $T_{\mathrm{bb}}$, die der entsprechenden Abbruchfrequenz $\nu^{*}$ gemäß Gleichung 1.15 entsprichen (siehe Text).

berechnet.

Da die hier vorgestellten Rechnungen mit einem Schwarzkörperspektrum durchgeführt werden und sich die Definition der Grenzfrequenz $\nu^{*}$ auf die Rayleigh-JeansApproximation bezieht, definieren wir für unsere Zwecke eine äquivalente Abruchfrequenz $\nu_{\mathrm{bb}}^{*}$ für ein Schwarzkörperspektrum der Temperatur $T_{\mathrm{bb}}$. Der integrierte Fluss ist durch $F_{\mathrm{bb}}=\sigma T_{\mathrm{bb}}^{4}$ gegeben und analog zu Gleichung 1.13 gilt

$$
\nu_{\mathrm{bb}}^{*}=\left(\frac{3 c^{2} \sigma}{2 \pi k}\right)^{1 / 3} T_{\mathrm{bb}}
$$

Die Schwarzkörpertemperaturen, die dieser Abbruchfrequenz entsprechen, sind in Abbildung 1.3 durch gestrichelte Linien dargestellt. Bei AM Her gilt $T \approx 20 \mathrm{keV}$, 


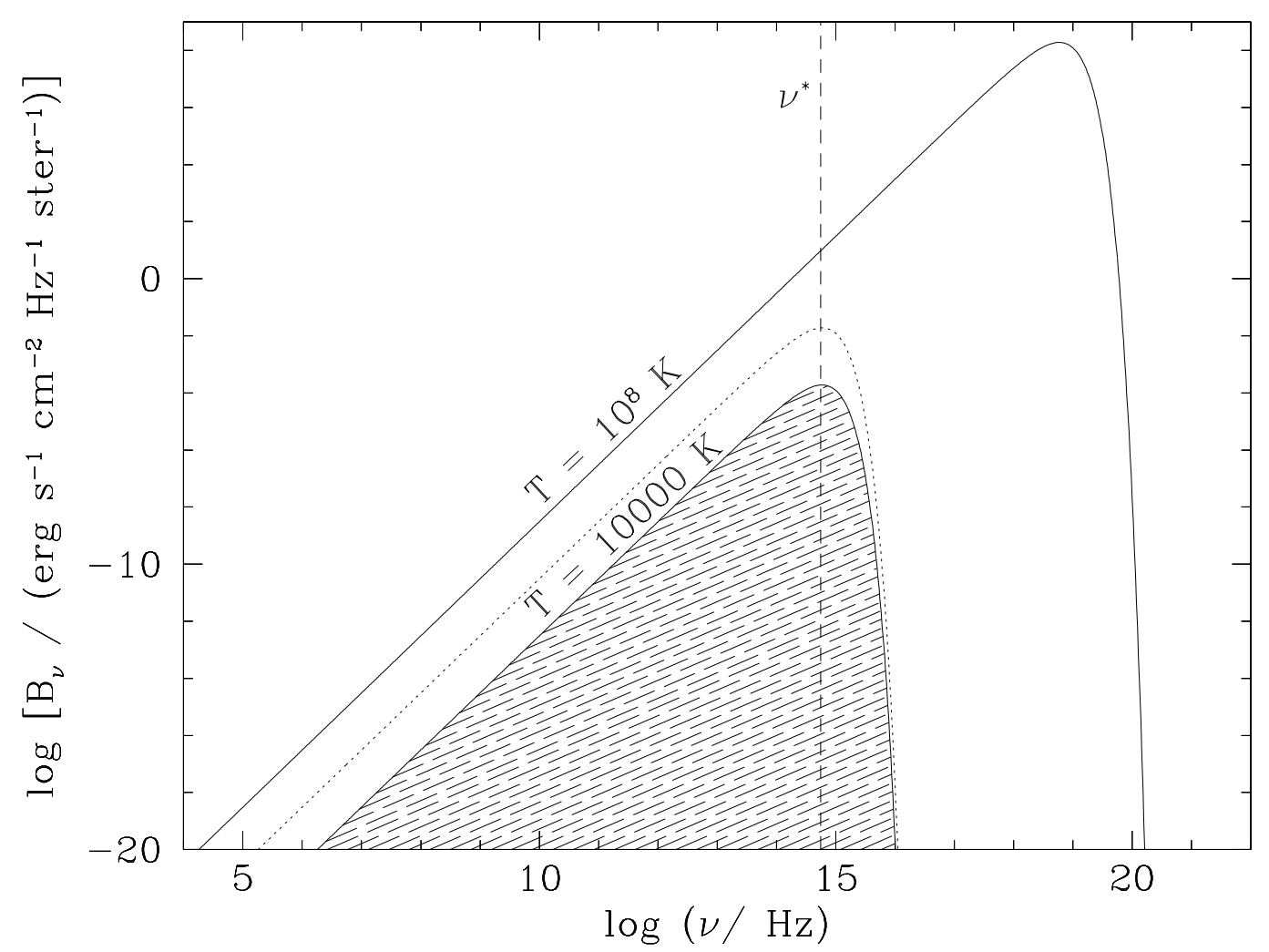

Abbildung 1.4: Modellierung der Zyklotronstrahlung durch einen Schwarzkörper. Der Rayleigh-Jeans-Bereich des Schwarzkörpers mit der Temperatur $T=10^{8} \mathrm{~K}$, der bei der durch die gestrichelte Linie markierten Frequenz $\nu^{*}$ abbricht, gilt als Referenz für den Fluss der Zyklotronstrahlung. Modelliert wird diese mit dem ausgefüllten Schwarzkörper der Temperatur $T=10000$ K. Dieser wird mit einem Skalierungsfaktor multipliziert, um verschiedene Bestrahlungsstärken zu modellieren (angedeutet durch den gepunkteten Schwarzkörper, der eine Skalierung mit dem Faktor 100 darstellt).

somit liegt die Abbruchfrequenz bei $\nu^{*} \approx 5 \cdot 10^{14} \mathrm{~Hz}$. Dies entspricht einem Schwarzkörper mit der Temperatur $T_{\mathrm{bb}} \approx 10000 \mathrm{~K}$.

Im Allgemeinen ist Kühlung durch Zyklotronstrahlung besonders effektiv bei hohen Temperaturen und geringen Dichten. Bei effektiver Zyklotronstrahlung ist wesentlich, dass die Strahlungsverluste der Elektronen stärker sind als der Energieaustausch zwischen den Ionen und den Elektronen. Es kommt zu einer Entkopplung der Ionen- und der Elektronentemperatur, so dass die hydrodynamische Behandlung nicht mehr als Einteilchengas erfolgen kann.

Es ist naheliegend, dass die Akkretionsregion neben ihrer Struktur in radialer Richtung eine Struktur bez. der Winkelvariablen besitzt. Dies ist in Abbildung 1.5 schematisch dargestellt. Danach besteht die Emissionsregion aus einem ausgedehn- 


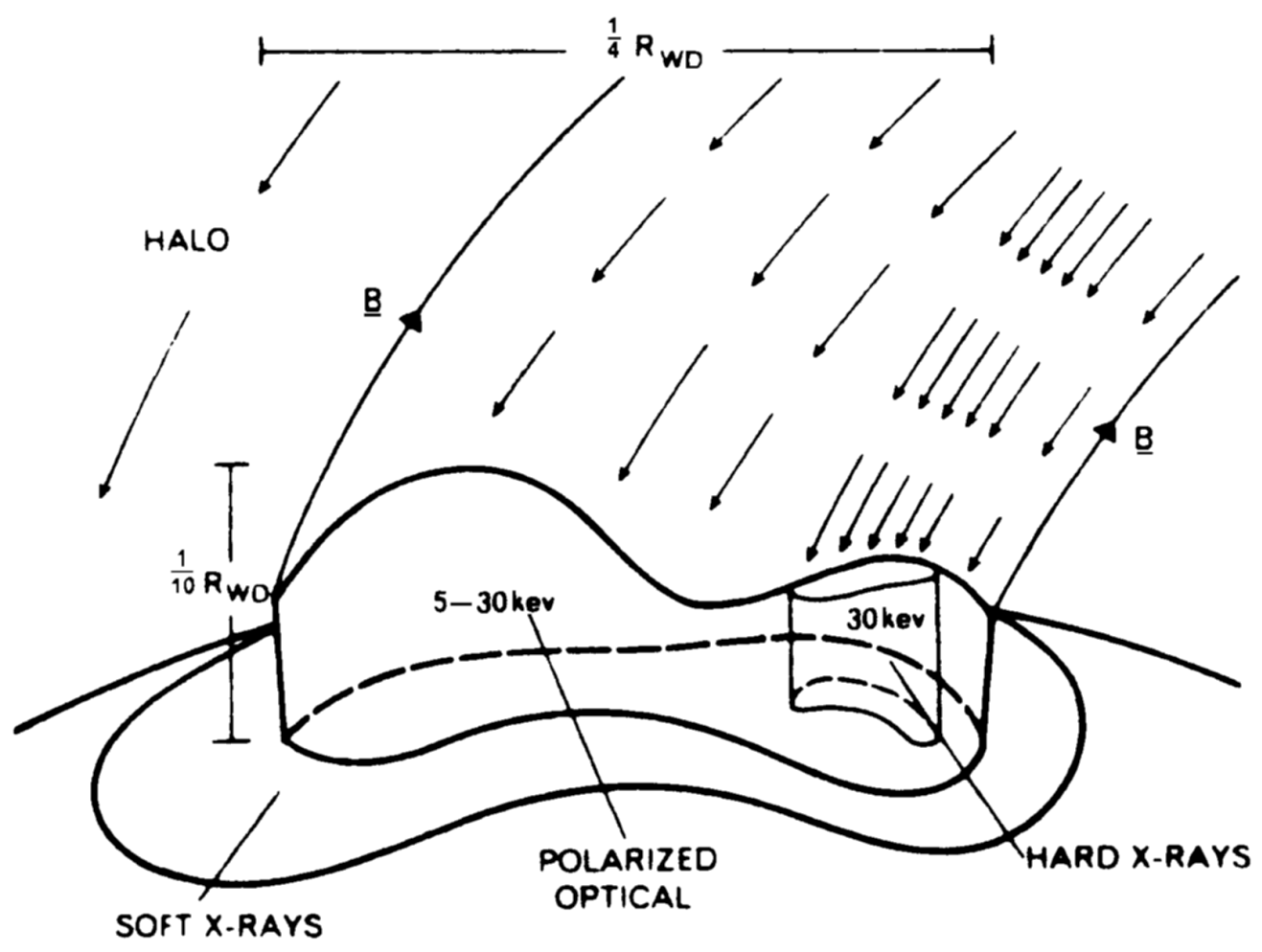

Abbildung 1.5: Schematische Darstellung einer ausgedehnten Akkretionsregion mit ortsabhängiger Akkretionsrate aus Warner (1995). Zu sehen ist ein Kern mit hoher Akkretionsrate, bei dem die Bremsstrahlung dominiert. Um den Kern herum existiert eine größere Region mit geringerer Akkretionsrate, in der hauptsächlich polarisierte Zyklotronstrahlung freigesetzt wird.

ten Volumen, bei dem die Akkretionsrate auf der Grundfläche variiert. Ein Bild wäre, dass es einen Kern mit hoher Akkretionsrate gibt, in dem harte Bremsstrahlung dominiert, während die Zyklontronstrahlung in einem größeren Bereich um den Kern herum entsteht.

Eine Abschätzung für die Akkretionsleuchtkraft ergibt sich, wenn man davon ausgeht, dass die gesamte kinetische Energie pro Fläche $d \tilde{E}_{\text {kin }}(\theta, \varphi)=\frac{1}{2} d m(\theta, \varphi) v_{\mathrm{ff}}^{2}$ der Massenflächendichte $d m$ am Ort $(\theta, \varphi)$ in Strahlungsenergie umgewandelt wird, dabei entspricht $v_{\mathrm{ff}}=\sqrt{2 G M / R}$ der Freifallgeschwindigkeit. Die Stromdichte (in radialer Richtung) ist dann $d m / d t=\dot{m}$. Die Gesamtleuchtkraft

$$
L=\int_{A} \frac{d \tilde{E}_{\mathrm{kin}}}{d t} d f=\frac{G M \dot{M}}{R}
$$

ergibt sich durch Integration über die Akkretionsfläche $A$, wobei $\dot{M}=\int_{A} \dot{m} d f$. Eine Schätzung der Akkretionsrate für AM Her im Zustand hoher Akkretion liefern Gänsicke et al. (2001) mit $\dot{M} \approx\left(10^{15}-10^{16}\right) \mathrm{g} \mathrm{s}^{-1}$. Damit liegt die Gesamtleuchtkraft 
bei $L \approx 10^{33} \mathrm{erg} \mathrm{s}^{-1}$. Der Anteil der Zyklontronstrahlung $L_{\text {cyc }}$ ist nur ein Teil der Gesamtleuchtkraft $L$. Zyklotronstrahlung dominiert bei niedrigen Massenflussraten und entsteht demnach in der Region um den Akkretionskern herum. Eine Abschätzung der Zyklotronleuchtkraft ist in Ramsay et al. (1994) mit $L_{\text {cyc }}=2.3 \cdot 10^{32} \mathrm{erg} \mathrm{s}^{-1}$ angegeben. Eine weitere Angabe des Zyklotronflusses von AM Her befindet sich bei Gänsicke et al. (1995) mit $F_{\text {cyc }}=1.8 \cdot 10^{-10} \mathrm{erg} \mathrm{s}^{-1} \mathrm{~cm}^{-2}$. Mit einem Abstand von $d=90$ pc ergibt dies - unter der Annahme, dass die Zyklotronstrahlung isotrop ist - eine Leuchtkraft von $L=1.7 \cdot 10^{32} \mathrm{erg} \mathrm{s}^{-1}$. Der Anteil der Zyklotronstrahlung am Gesamtstrahlungsfluss in Abhängigkeit von der Massenflussrate wurde in Woelk \& Beuermann (1996) mit einem eindimensionalen stationären hydrodynamischen Zweiflüssigkeitenmodell berechnet. Dabei ist bei einem Weißen Zwerg der Masse $M=0.7 M_{\odot}$ mit einem Magnetfeld $B \approx 15 \mathrm{MG}$ der Anteil der Zyklotronstrahlung $F_{\text {cyc }} / F>90 \%$ für $\dot{m}<10^{-2} \mathrm{~g} \mathrm{~cm}^{-2} \mathrm{~s}^{-1}$, bei zunehmender Massenstromdichte $\dot{m}$ nimmt der Anteil ab, liegt bei $\dot{m}=1 \mathrm{~g} \mathrm{~cm}^{-2} \mathrm{~s}^{-1}$ bei ca. $40 \%$, um bei $\dot{m}>10 \mathrm{~g} \mathrm{~cm}^{-2} \mathrm{~s}^{-1}$ auf unter $10 \%$ zu fallen.

\subsection{Beobachtungen von AM Her}

Das System AM Her gilt als Prototyp der Polare. Der Name entspringt der Beobachtung, dass linear und zirkular polarisierte Strahlung vom optischen bis zum infraroten Spektralbereich charakteristisch für diesen Sterntyp ist. Die Existenz polarisierter Strahlung läßt auf ein starkes Magnetfeld beim Weißen Zwerg schließen. Die Analyse der Intensitätmodulation als Funktion der Orbitalphase des Systems zeigt, dass diese aus der Umgebung der magnetischen Polkappen des Weißen Zwerges stammen muss. Dass es sich bei dieser Strahlung um Zyklotronstrahlung handelt, läßt sich aus der direkten Beobachtung der Harmonischen folgern. Die Elektronentemperaturen, die sich aus den Zyklotronspektren ableiten lassen, lassen darauf schließen, dass die Strahlung in der Umgebung der Region ensteht, die mit der hydrodynamischen Stoßwelle assoziiert ist (Wickramasinghe \& Meggitt, 1985).

Polare zeigen eine Variation der Leuchtkraft auf großer Zeitskala ( $\approx$ Monate) in unregelmäßigen Intervallen. Diese Variation ist nicht korreliert mit der Variation, die durch die Orbitalbewegung (die Perioden liegen im Bereich $P_{\text {orb }} \approx 1-5 \mathrm{~h}$, bei AM Her liegt eine Orbitalperiode von $P_{\text {orb }}=3.09$ min vor, Ritter \& Kolb 1998) zu erwarten ist. Da sich bei Polaren keine Akkretionsscheibe ausbildet, korrespondiert die Leuchtkraft direkt mit der Akkretionsrate. Daher ist diese Variation Ausdruck einer Änderung des Massenstroms $\dot{M}$. Den Zustand schwacher Leuchtkraft nennt man auch Low State, den Zustand starker Leuchtkraft High State. Bei AM Her entsprechen die Zustände einer visuellen Leuchkraft von $m_{V}[\mathrm{Hi}]=12^{m}$ und $m_{V}[\mathrm{Lo}]=15^{m} \cdot 5$ (Hessman et al., 2000).

Zur genauen Analyse der Spektren bzw. Lichtkurven des Systems ist zu berücksichtigen, dass die beobachtete Strahlung an verschiedenen Orten des Sytems durch verschiedene physikalische Prozesse entsteht: 
- In der Atmosphäre des Weißen Zwerges: Bei einer angenommenen Effektivtemperatur von $T_{\text {eff }}=20000 \mathrm{~K}$ liegt das Maximum des Spektrum des Weißen Zwerges bei $\lambda_{\max } \approx 1100 \AA$. Im UV Bereich $1150 \AA \lesssim \lambda \lesssim 1400 \AA$ dominieren die Beiträge des Weißen Zwerges, so dass andere Beiträge eine marginale Rolle spielen. Einzelne Frequenzbänder dieses Bereichs sind in den Lichtkurven in Abbildung 1.6 dargestellt.

- Im Sekundärstern: Der Sekundärstern von AM Her wird identifiziert als Zwergstern vom Spektraltyp dM4.5/5 (Ritter \& Kolb, 1998). Bei einer Effektivtemperatur von $T_{\text {eff }} \approx 3000 \mathrm{~K}$ liegt das Schwarzkörpermaximum bei $\lambda_{\max } \approx 10000 \AA$.

- Im Akkretionsstrom: Der nahe UV Bereich bei $\lambda \gtrsim 2000 \AA$ ist im High State dominiert von der Kontinuumsemission des Akkretionsstroms. Der Stromanteil wurde in Gänsicke et al. (1995) modelliert mit einem Schwarzkörper der Temperatur von $T=10000 \mathrm{~K}$. Dabei ist jedoch unsicher, welchen Anteil der Strom im Bereich $\lambda<2000 \AA$ besitzt. Es wäre denkbar, dass der Anteil des Stroms in diesen Bereich weniger stark abfällt.

- In der Akkretionsregion: Der Akkretionsstrom trifft in der Nähe des (der) magnetischen Pols (Pole) auf die Oberfläche des Weißen Zwerges. Dort bildet sich eine Stoßwelle aus, in der die Materie auf $\approx 10^{8} \mathrm{~K}$ erhitzt wird. Hier entsteht einerseits harte Bremsstrahlung im Bereich von $2-60 \mathrm{keV}$ und andererseits Zyklotronstrahlung im optischen und infraroten Bereich. Beide Strahlungen treffen auf die Oberfläche des Weißen Zwerges und sind verantwortlich für die Aufheizung der Atmosphäre.

Die Abbildungen 1.6 und 1.7 zeigen Beobachtungen von AM Her im High State, die 1997 mit dem Goddard High Resolution Spectrograph (GHRS) auf dem Hubble Space Telescope (HST) aufgenommen wurden. Eine Analyse der Daten wird in Gänsicke et al. (1998) präsentiert.

Abbildung 1.6 zeigt den zeitabhängigen Kontinuumsfluss von AM Her in ausgewählten Frequenzbereichen der UV-Region. Es zeigt sich eine phasenabhängige sinusförmige Modulation, von der angenommen wird, dass sie durch die Existenz einer geheizten Region der Atmosphäre des Weißen Zwerges, die durch die in der Akkretionsregion entstehende Strahlung bestrahlt wird, hervorgerufen wird. Dabei wird in der Darstellung die Orbitalphase $\varphi_{\text {orb }}$ benutzt. Diese definiert $\varphi_{\text {orb }}=0$ durch die untere Konjunktion des Sekundärsterns. Sie unterscheidet sich von der häufig verwendeten magnetischen Phase $\varphi_{\text {mag }}$, die das Ereignis der maximalen linearen Polarisation mit $\varphi_{\text {mag }}=0$ identifiziert. Zur Phase $\varphi_{\text {mag }}=0$ ist der Sichtwinkel zur Akkretionssäule maximal. Bei AM Her gilt zwischen den beiden Phasenkonventionen die Relation $\varphi_{\text {orb }}=\varphi_{\text {mag }}+0.367$. Die durchgezogenen Linien stellen simulierte Modell-Lichtkurven dar. Bei diesem Modell wurde ein heißer Fleck mit vorgegebener Struktur angenommen. Ein durch Flächenelemente dargestellter Weißer Zwerg mit 


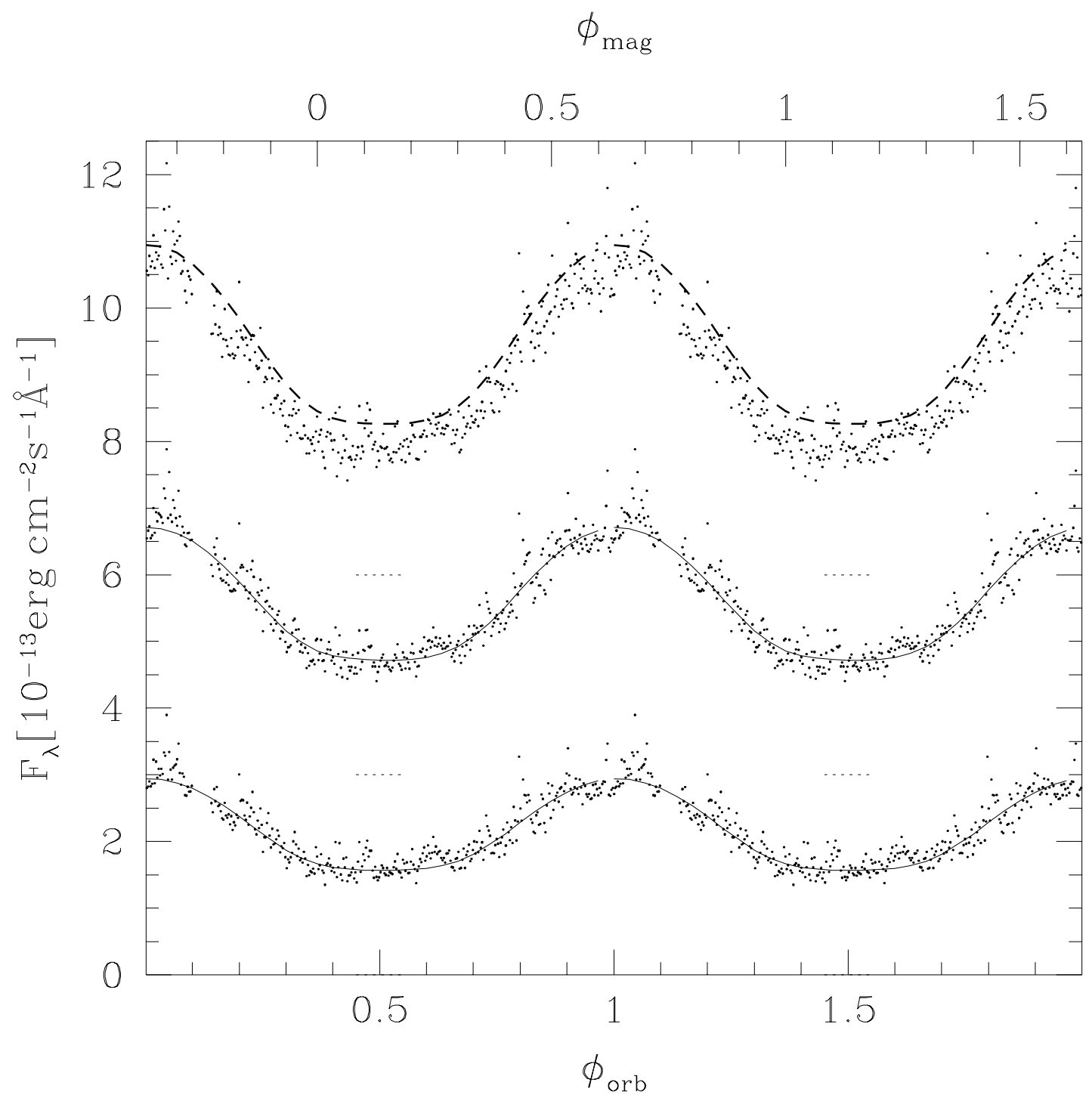

Abbildung 1.6: Modulation der Lichtkurve im UV-Spektralbereich aus Gänsicke et al. (1998). Die einzelnen Kurven zeigen verschiedene Wellenlängenbereiche: Von oben nach unten $1150 \AA \leq \lambda \leq 1167 \AA, 1254 \AA \leq \lambda \leq 1286 \AA$ und $1412 \AA \leq \lambda \leq 1427 \AA$. Die gestrichelten Markierungen kennzeichnen den jeweiligen Nullpunkt auf der Ordinatenachse.

gegebener Effektivtemperatur $T_{\text {eff }}$ und Oberflächenschwerebeschleunigung $g$ wird überlagert mit Flächenelementen, die den Fleck repräsentieren. Jedem einzelnen Flächenelement wurde eine Effektivtemperatur zugewiesen. Die Struktur des Flecks wurde dabei a priori angenommen. Die Verteilung wurde als kreisförmige Fläche mit vom Mittelpunkt linear abnehmender Temperatur modelliert. Die Synthese der Lichtkurven und Spektren erfolgte durch die Zuordnung von Modellspektren an die 


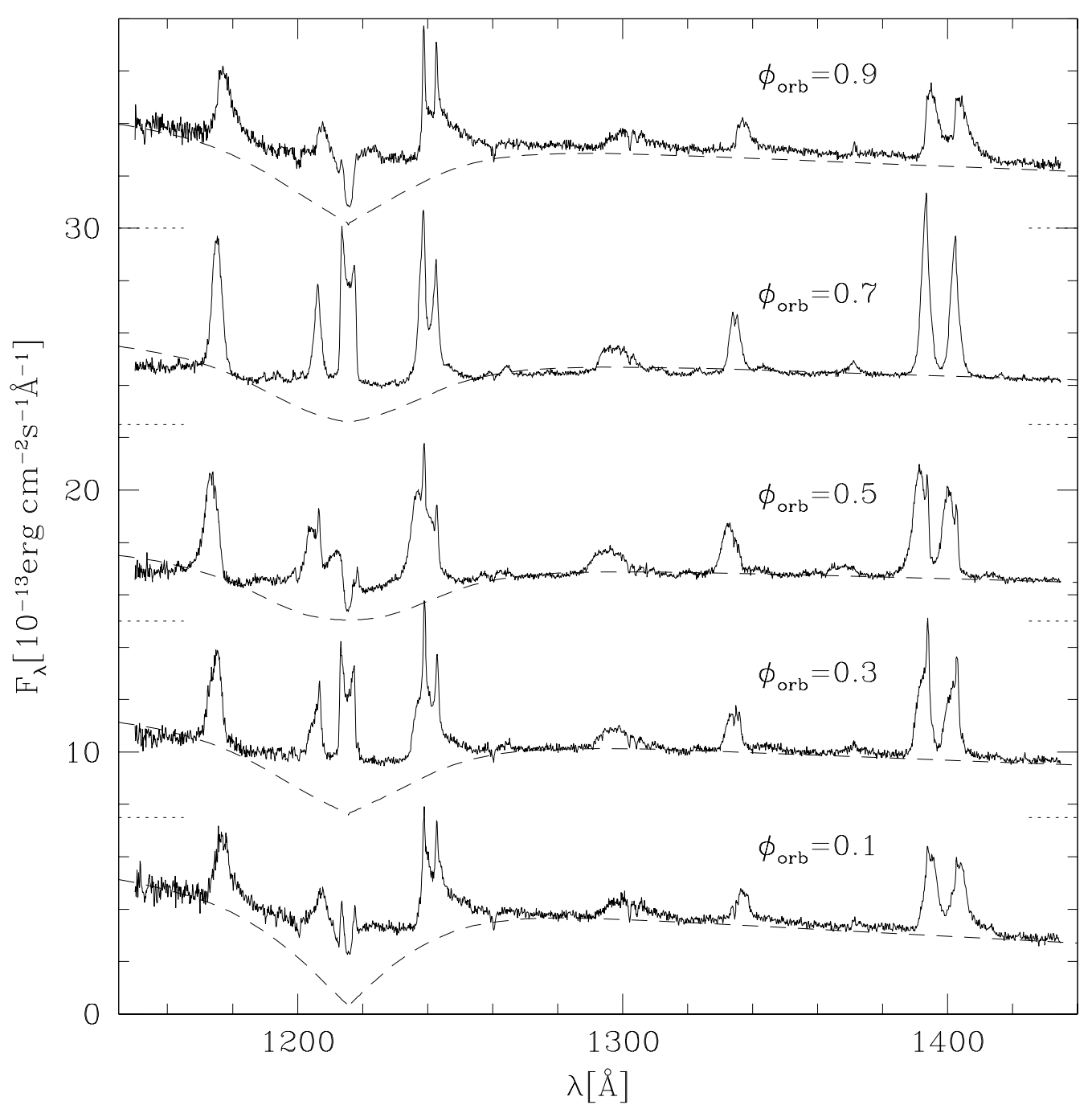

Abbildung 1.7: Phasenaufgelöste Spektren von AM Her im Zustand hoher Akkretionsrate (High State) aus Gänsicke et al. (1998). Die gestrichelten Kurven repräsentieren die Spektren eines einfachen Modells, welches den geheizten Fleck durch unbestrahlte Atmosphärenmodelle verschiedener Effektivtemperatur zusammensetzt. Signifikant ist, dass die breite Ly $\alpha$-Linie stark aufgefüllt ist.

jeweiligen Flächenelemente.

In Abbildung 1.7 sind die phasenabhängigen Spektren von AM Her im Bereich $1150 \AA \leq \lambda \leq 1450 \AA$ dargestellt. Die Spektren zeigen das System im High State. Die hier sichtbare Strahlung besteht aus verschiedenen Komponenten: Es dominiert die Strahlung des Weißen Zwerges, diesem Spektrum ist ein Emissionslinienspektrum 
überlagert, das aus dem Akkretionsstrom des Systems stammt. Unklar ist hierbei, wieviel der Kontinuumsanteil aus dem Akkretionsstrom in diesem Bereich beiträgt. Weiterhin ist im Bereich der Ly $\alpha$-Linie bei $\lambda=1216 \AA$ einerseits Emission zu erkennen, die allerdings geokoronalen Ursprungs ist und nicht aus dem System stammt. Dies ist überlagert von einem schmalen interstellarer Ly $\alpha$-Absorptionskern.

Wesentlich ist, dass die für einen Weißen Zwerg charakteristischen breiten Absorptionslinien - hier die Ly $\alpha$-Linie - fast vollständig fehlen (Raymond et al., 1995; Mauche \& Raymond, 1998; Greeley et al., 1999). Im Low State hingegen, wo die Absorptionslinien vorhanden sind, sind die Daten kompatibel mit den Modellen Weißer Zwerge und erlauben eine Identifikation des Weißen Zwerges mit einer Effektivtemperatur $T_{\text {eff }} \approx 20000 \mathrm{~K}$ und $\log \left[g /\left(\mathrm{cm} \mathrm{s}^{-2}\right)\right] \approx 8$ durch das Profil der Ly $\alpha$-Linie (Gänsicke et al., 1995).

Die gestrichelten Linien in Abbildung 1.7 zeigen Modellrechnungen. Der Fleck, der vermutlich durch die freigesetzte Strahlung aus der Umgebung der Stoßwellenregion geheizt wird, wurde dabei einfach durch Modifikation der Effektivtemperatur $T_{\text {eff }}$ von normalen Modellatmosphären simuliert. Es wurde also angenommen, dass das Spektrum eines bestrahlten Weißen Zwerges dem Spektrum eines nicht bestrahlten Weißen Zwerges mit etwas höherer Effektivtemperatur ähnlich ist. Dies ist eine Annahme, die kritisch ist, da sich die Struktur einer bestrahlten Atmosphäre - wie noch gezeigt werden wird - wesentlich von derjenigen einer unbestrahlten Atmosphäre unterscheidet. Weiterhin zeigen die Rechnungen von Modellatmosphären in Abbildung 1.8, dass eine ungestörte nicht-bestrahlte Atmosphäre zwar eine Modulation des Profils der Lyman-Linien zeigt, jedoch keine signifikante Auffüllung, die die Beobachtungen in 1.7 erklären könnte. Dieser Aspekt wurde schon in Gänsicke et al. (1998) vermutet, und es wurden Rechnungen durchgeführt, wie eine modifizierte Temperaturschichtung das Spektrum verändern könnte. Dabei wurden Temperaturschichtungen $T(\tau)$ a priori angenommen und folgten nicht aus einer konsistenten Modellierung durch den Atmosphärencode.

Man erwartet, dass die in der Akkretionsregion freigesetzte Strahlung eine größere Region der Atmosphäre des Weißen Zwerges aufheizt. Die Frage ist dabei, wie die modifizierte Struktur des Temperaturverlaufs, der sich aus einem konsistenten Atmosphärenmodell ergibt, den Strahlungsfluss verändert. Da in den beobachteten Spektren von AM Her die Absorption in der Ly $\alpha$-Linie nahezu verschwindet, wäre es wünschenswert zu erfahren, unter welchen Bedingungen ein Verschwinden der Absorption im Modell simuliert werden kann.

\subsection{Aufbau der Arbeit}

Es ist Ziel der Arbeit, im Hinblick auf die AM Her-Systeme ein Modell eines Weißen Zwergs zu entwickeln, der von einer externen Quelle bestrahlt wird.

Dazu wird in Kapitel 2 zunächst der zugrundeliegende Atmosphärencode at beschrieben, der Ausgangspunkt für die Atmosphärenmodelle ist. Dieser Code wur- 


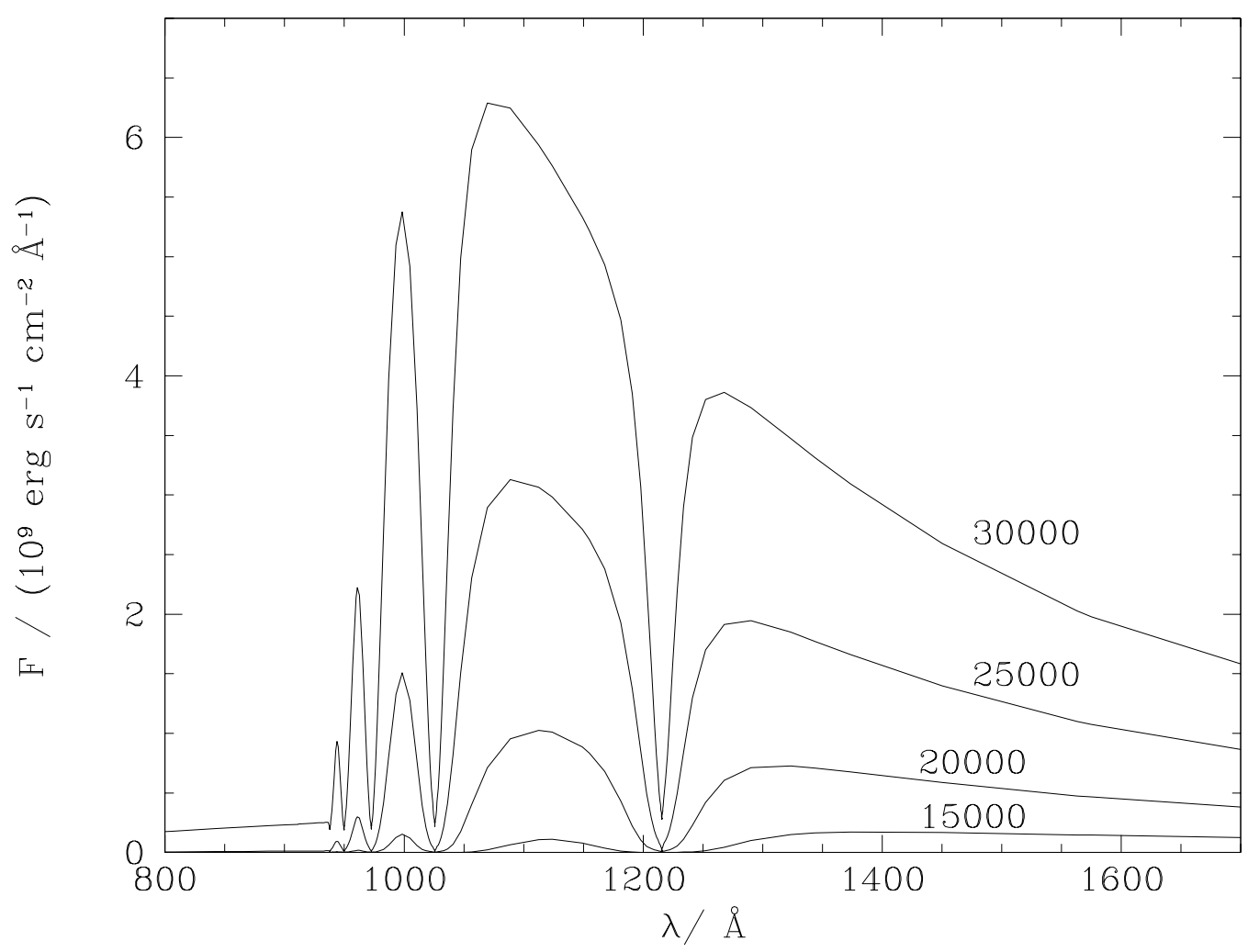

Abbildung 1.8: Spektren von Atmosphärenmodellen mit $\log \left[\mathrm{g} /\left(\mathrm{cm} \mathrm{s}^{-2}\right)\right]=8$ und verschiedenen Effektivtemperaturen $T_{\text {eff }} / \mathrm{K}$.

de erweitert, um Atmosphären mit externer Bestrahlung berechnen zu können. Die Bestrahlung wird zunächst isotrop angesetzt. Darauf folgt die Betrachtung winkelabhängiger Bestrahlung mit einem größeren Winkelgitter. Auf dieser Grundlage wird ein Gitter von Modellatmosphären mit verschiedenen Bestrahlungswinkeln und Bestrahlungsflüssen berechnet.

In Kapitel 3 wird dieses Modellatmosphärengitter benutzt, um aus den eindimensionalen planparallelen Modellatmosphären ein dreidimensionales Modell eines Weißen Zwerges mit einer externen Bestrahlungsquelle zusammenzusetzen. Dazu wird die Oberfläche in eine Anzahl von Flächenelementen zerlegt, denen jeweils ein Atmosphärenmodell, welches sich aus der definierten Bestrahlungsquelle bestimmen lässt, zugeordnet wird. Daraus wird das phasenabhängige Gesamtspektrum der sichtbaren Oberfläche synthetisiert. Es folgt eine Beschreibung der Ergebnisse für verschiedene Geometrien und Leuchtkräfte der Emissionsregion.

In Kapitel 4 wird versucht, das Modell auf die verfügbaren HST/GHRS-Beobachtungen von AM Her anzuwenden. Es liegen phasenaufgelöste Spektren vor, die neben einer Modulation des Flusses eine signifikant aufgefüllte Ly $\alpha$ Linie zeigen. Es wird versucht, diese Beobachtungen mit dem Modell zu beschreiben. 


\section{Kapitel 2}

\section{Atmosphärenmodellierung mit Einstrahlung}

\subsection{Atmosphärenmodell}

\subsubsection{Grundlagen}

Unter der Atmosphäre eines Sterns versteht man die äußeren Gasschichten, die für die spektrale Form des Strahlungsflusses verantwortlich sind. Die im Inneren des Sterns erzeugte bzw. vorhandene Strahlungsenergie wird durch die Schichten der Atmosphäre nach außen transportiert. Durch die Wechselwirkung von Strahlung und Materie erhält der Strahlungsfluss die Form, in der er als Spektrum beobachtbar ist. Ziel der Atmosphärenmodellierung ist es, Aussagen über den Strahlungsfluss und den Zustand der Materie zu treffen. Als Grundlage benutzen wir dabei den Atmosphärencode at, wie er in Gänsicke (1993) dargestellt ist. Die zugrundeliegenden Annahmen des Codes machen ihn vor allem für die Anwendung auf die Atmosphären Weißer Zwerge geeignet:

- Materiezusammensetzung aus reinem Wasserstoff: In den hier betrachteten Weißen Zwergen vom Typ DA sind fast ausschließlich Wasserstofflinien sichtbar.

- Annahme von lokalem thermodynamischen Gleichgewicht (LTE):

Für unbestrahlte Weiße Zwerge mit einer Effektivtemperatur von $T_{\text {eff }}=20000 \mathrm{~K}$ und $\log \left[\mathrm{g} /\left(\mathrm{cm} \mathrm{s}^{-2}\right)\right]=8$ sind die Abweichungen von NLTE-Modellen vernachlässigbar. Atmosphären aus reinem Wasserstoff zeigen Abweichungen ab $T_{\text {eff }} \gtrsim 80000 \mathrm{~K}$ (Napiwotzki, 1997). Dabei muss jedoch beachtet werden, dass diese Werte auf unbestrahlten Modellen beruhen. Da die Bestrahlung zu einer Erhitzung von Schichten geringer optischer Tiefe führt, sind NLTE Effekte vor allem in den Linienkernen zu erwarten.

- Behandlung von isotroper kohärenter Thomson-Streuung. 
- Reduktion der Geometrie auf ein eindimensionales planparalleles Schichtenmodell: Das Verhältnis der Ausdehnung der Atmosphäre zum Radius ist relativ gering $\left(\approx 10^{-4}-10^{-3}\right)$, so dass sphärische Effekte vernachlässigbar sind.

Die vorgegebenen Modellparameter sind bei at gegeben durch

- die Effektivtemperatur $T_{\text {eff }}$

- die Oberflächenschwerebeschleunigung $g$.

at soll erweitert werden, so dass eine Behandlung von bestrahlten Atmosphären möglich ist. Daher muss der Modellparametersatz erweitert werden um die Spezifikation der Intensität der einfallenden Strahlung in Abhängigkeit von Frequenz $\nu$ und Winkel $\theta$. Die erweiterte Version des Codes bezeichnen wir mit atX.

\subsubsection{Geometrie}

Die Geometrie des Atmosphärenmodells besteht aus planparallelen Schichten, wie in Abbildung 2.1 dargestellt. Die Zustandsvariablen einer Schicht sind jeweils konstant. Für eine Größe $A(x, y, z)$ gilt

$$
\frac{\partial A}{\partial x}=0, \quad \frac{\partial A}{\partial y}=0
$$

Die Ortsabhängigkeit der physikalischen Größen ist somit reduziert auf eine Dimension. Weiterhin wird die Abhängigkeit von der Richtung, in der parametrischen Darstellung durch die Winkelkoordinaten $(\theta, \varphi)$ gegeben durch

$$
\vec{n}=(\sin \theta \cos \varphi, \sin \theta \sin \varphi, \cos \theta)
$$

reduziert auf die Abhängigkeit von $\theta$. Die Intensität ist somit rotationssymmetrisch bez. $\varphi$. Eine häufig verwendete Konvention ist die Benutzung von $\mu$ für den Winkelkosinus:

$$
\mu:=\cos \theta
$$

Zusätzlich wird im Modell keine Zeitabhängigkeit betrachtet.

\subsubsection{Strahlungstransport}

Die physikalische Modellierung der Atmosphäre erfordert die Beschreibung des Zustands der Materie, der Strahlung und ihrer gegenseitigen Wechselwirkung. Dazu ist die Einführung einiger Größen notwendig. 
aussen

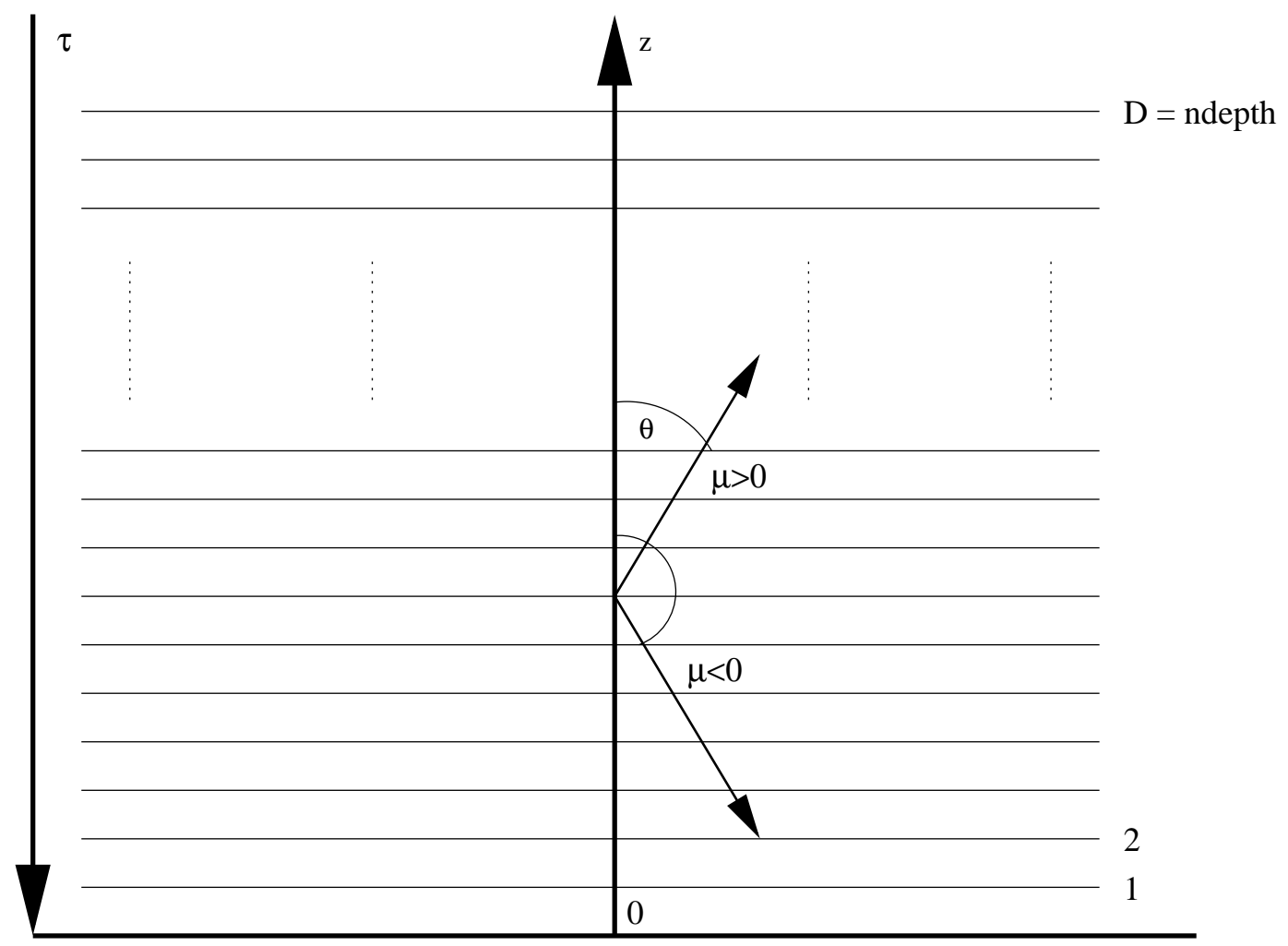

innen

Abbildung 2.1: Schematische Darstellung des eindimensionalen Schichtenmodells der Atmosphärengeometrie. Die geometrische Tiefe $z$ nimmt von innen nach außen zu. Die optische Tiefe $\tau$ hingegen beschreibt die Durchsichtigkeit der Materie und wird in (2.27) definiert. Die Richtungsangabe erfolgt ausgehend vom Normalenvektor in positiver $z$-Richtung durch den Winkel $\theta$. Oft wird die Größe $\mu=\cos \theta$ verwendet. Für ausgehende Strahlen gilt dann $\mu>0$ und für einfallende $\mu<0$.

Intensität Die elementare Größe zur makrospkopischen Beschreibung von Strahlung am Ort $\vec{r}$, in Richtung $\vec{n}$, der Frequenz $\nu$ zur Zeit $t$ ist die Intensität $I(\vec{r}, \vec{n}, \nu, t)$. Sie ist so definiert, dass die Gesamtenergie $E$ durch eine Fläche $A$ im Zeitintervall $\Delta t=t_{1}-t_{0}$ gegeben ist $\operatorname{durch}^{1}$

$$
E=\int_{t_{0}}^{t_{1}} d t \int_{0}^{\infty} d \nu \int_{\mathcal{S}^{2}} d f \int_{A} I(\vec{r}, \vec{n}, \nu, t) \vec{n} \cdot d \vec{f}
$$

1 In den Integralen bezeichnet $d f$ das skalare und $d \vec{f}$ das vektorielle Flächenelement. $\mathcal{S}^{2}=\left\{\vec{x} \in \mathbb{R}^{3}:|\vec{x}|=1\right\}$ ist die Einheitssphäre. 
Die Einheit der Intensität im cgs-System ist demnach $\operatorname{erg~s}^{-1} \mathrm{~cm}^{-2} \mathrm{sr}^{-1} \mathrm{~Hz}^{-1}$. Im Schichtenmodell ohne Zeitabhängigkeit gilt für die Intensität $I$ :

$$
I(\vec{r}, \vec{n}, \nu, t) \equiv I(z, \mu, \nu) .
$$

Mittlere Intensität Die mittlere Intensität $J$ ist die über alle Richtungen gemittelte Intensität:

$$
J(\vec{r}, \nu, t):=\frac{1}{4 \pi} \int_{\mathcal{S}^{2}} I(\vec{r}, \vec{n}, \nu, t) d f
$$

Im Schichtenmodell gilt:

$$
J(z, \nu)=\frac{1}{2} \int_{-1}^{1} I(z, \mu, \nu) d \mu .
$$

Fluss Der Fluss $\vec{F}$ ist definiert durch

$$
\vec{F}(\vec{r}, \nu, t):=\int_{\mathcal{S}^{2}} I(\vec{r}, \vec{n}, \nu, t) \vec{n} d f .
$$

Man beachte, dass der Fluss eine vektorielle Größe ist. Im Schichtenmodell verschwinden die Komponenten $F_{x}$ und $F_{y}$. Der Fluss $\vec{F}$ kann hier also mit der Komponente $F_{z}$ identifiziert werden. Es gilt dann:

$$
F(z, \mu, \nu)=2 \pi \int_{-1}^{1} I(z, \mu, \nu) \mu d \mu .
$$

Die Bedeutung des Flussbegriffs wird offensichtlich, wenn man (2.4) folgendermaßen schreibt:

$$
E=\int_{t_{0}}^{t_{1}} d t \int_{0}^{\infty} d \nu \int_{A} \vec{F}(\vec{r}, \nu, t) \cdot d \vec{f}
$$

Der Fluss entspricht dem Begriff der Stromdichte einer bestimmten Frequenz $\nu$.

Für die Flussdefinition gibt es noch verschiedene alternative Konventionen, die sich lediglich durch einen Vorfaktor unterscheiden.

- Astrophysikalischer Fluss $F_{\text {ap }}$ :

$$
F_{\text {ap }}(\vec{r}, \nu, t):=\frac{1}{\pi} F(\vec{r}, \nu, t)
$$

- Eddington-Fluss $H$ :

$$
H(\vec{r}, \nu, t):=\frac{1}{4 \pi} F(\vec{r}, \nu, t)
$$


Strahlungsdrucktensor Die Größe

$$
\mathbf{P}:=\frac{1}{c} \int_{\mathcal{S}^{2}} I(\vec{r}, \vec{n}, \nu, t) \vec{n} \cdot \vec{n}^{t} d \omega
$$

definiert den Strahlungsdrucktensor2. Er ist symmetrisch und besitzt im Fall der vorliegenden Geometrie eine Diagonalform, die durch die Angabe der skalaren Größen

$$
\begin{aligned}
E_{\mathrm{rad}}(z, \mu, \nu) & :=\frac{4 \pi}{c} J(z, \mu, \nu) \\
p_{\mathrm{rad}}(z, \mu, \nu) & :=\frac{4 \pi}{c} K(z, \mu, \nu)
\end{aligned}
$$

bestimmt ist, wobei $K$ definiert ist durch

$$
K(z, \mu, \nu):=\frac{1}{2} \int_{-1}^{1} I(z, \mu, \nu) \mu^{2} d \mu .
$$

Für das Modell interessiert im wesentlichen die Kraftdichte, die durch die Strahlung auf ein Volumen wirkt. Diese hängt lediglich von dem Divergenzausdruck ${ }^{3}$

$$
\nabla \mathbf{P} \equiv \frac{\partial P^{i j}}{\partial x^{j}} \vec{e}_{i}
$$

ab, welcher sich in unserem Fall auf den Ausdruck

$$
\frac{\partial p_{\mathrm{rad}}(z, \nu)}{\partial z}=\frac{4 \pi}{c} \frac{\partial K(z, \nu)}{\partial z}
$$

reduziert.

Wechselwirkung von Strahlung und Materie Bewegt sich Strahlung durch ein Medium, so unterliegt sie der Wechselwirkung mit der Materie. Beschrieben wird diese Interaktion durch den Absorptions- und Emissionskoeffizienten. Die Opazität $\chi$ beschreibt den Verlust der Energie eines Strahls, der ein Volumen V durchdringt.

$$
\delta E=\chi(\vec{r}, \vec{n}, \nu, t) I(\vec{r}, \vec{n}, \nu, t) d V d \omega d \nu d t .
$$

$\chi$ kann aufgespalten werden in einen Teil $\kappa$, der Absorption beschreibt und einen Teil $\sigma$, der Streuung beschreibt:

$$
\chi(\vec{r}, \nu, t)=\kappa(\vec{r}, \nu, t)+\sigma(\vec{r}, \nu, t),
$$

Weiterhin beschreibt der Emissionskoeffizient $\eta$ den Anteil der Strahlungsenergie, der von einem Materievolumen freigesetzt wird:

$$
\delta E=\eta(\vec{r}, \vec{n}, \nu, t) d V d \omega d \nu d t .
$$

Im thermischen Gleichgewicht gilt das Kirchhoff-Plancksche Gesetz $\eta=\kappa B$. In unserem Fall setzt sich die Emissivität aus einem Streuanteil $\eta_{\mathrm{s}}=\sigma J$ und einem thermischen Anteil $\eta_{\mathrm{t}}=\kappa B$ zusammen.

\footnotetext{
${ }^{2} \vec{n}^{t}$ bezeichnet den transponierten Vektor. Wenn $\vec{n}$ ein Spaltenvektor ist, ist $\vec{n}^{t}$ ein Zeilenvektor und das Produkt $\vec{n} \cdot \vec{n}^{t}$ eine Matrix.

${ }^{3}$ Summation erfolgt über je einen gleichen Index oben und unten, wobei ein oberer Index unter dem Bruchstrich als unterer Index interpretiert wird.
} 
Strahlungstransportgleichung Die Strahlungstransportgleichung beschreibt den Zusammenhang zwischen dem Materiezustand und dem Strahlungsfeld:

$$
\left[\frac{1}{c} \frac{\partial}{\partial t}+\vec{n} \cdot \vec{\nabla}\right] I(\vec{r}, \vec{n}, \nu, t)=\eta(\vec{r}, \vec{n}, \nu, t)-\chi(\vec{r}, \vec{n}, \nu, t) I(\vec{r}, \vec{n}, \nu, t) .
$$

Im stationären Schichtenmodell gilt

$$
\frac{\partial I}{\partial x}=0 \quad, \quad \frac{\partial I}{\partial y}=0 \quad \text { und } \quad \frac{\partial I}{\partial t}=0 .
$$

Somit reduziert sich die Strahlungstransportgleichung in unserem Fall $\left(n_{z}=\cos \theta=\mu\right)$ auf

$$
\begin{aligned}
\mu \frac{\partial I(z, \mu, \nu)}{\partial z} & =\eta(z, \nu)-\chi(z, \nu) I(z, \mu, \nu) \\
& =-\chi(z, \nu)[I(z, \mu, \nu)-S(z, \nu)],
\end{aligned}
$$

wobei bei der Umformung die Ergiebigkeit (engl. source function)

$$
S(z, \nu):=\frac{\eta(z, \nu)}{\chi(z, \nu)}
$$

eingeführt wurde. Im Modell ist die Ergiebigkeit gegeben durch

$$
S(z, \nu)=\frac{\kappa(z, \nu)}{\chi(z, \nu)} B(z, \nu)+\frac{\sigma(z, \nu)}{\chi(z, \nu)} J(z, \nu) .
$$

Sie besteht aus einem thermischen Anteil $(\kappa / \chi) B$ und einem Streuanteil $(\sigma / \chi) J$, der die kohärente und isotrope Thomson-Streuung beschreibt. Definiert man sich noch die optische Tiefe $\tau$ durch

$$
\tau(z, \nu):=\int_{z}^{z_{\max }} \chi\left(z^{\prime}, \nu\right) d z^{\prime}
$$

läßt sich die Strahlungstransportgleichung noch auf ihre einfachste Form bringen 4

$$
\mu \frac{\partial I(\tau, \mu, \nu)}{\partial \tau}=I(\tau, \mu, \nu)-S(\tau, \nu)
$$

Zur Lösung der Strahlungstransportgleichung Bei vollständig bekannter Ergiebigkeit $S(z, \nu)$ ließe sich die Strahlungstransportgleichung direkt integrieren, da sie sich durch Multiplikation mit dem integrierenden Faktor $e^{-\tau / \mu}$ auf folgende Form bringen läßt:

$$
\frac{\partial}{\partial \tau}\left[I(\tau, \mu, \nu) e^{-\tau / \mu}\right]=-\frac{S(\tau, \nu)}{\mu} e^{-\tau / \mu}
$$

\footnotetext{
${ }^{4}$ Genaugenommen handelt es sich bei $I(\tau, \mu, \nu)$ und $S(\tau, \nu)$ um neue Funktionen $\tilde{I}$ und $\tilde{S}$, die $\operatorname{durch} \tilde{I}(\tau, \mu, \nu):=I(z, \mu, \nu)$ definiert sind (analog für $\tilde{S})$
} 
Im Allgemeinen ist die Ergiebigkeit aber nicht bekannt, sondern hängt selbst in integraler Form vom Strahlungsfeld $I$ ab, so dass die formale Lösung nur zusammen mit iterativen Verfahren zu einer Lösung führt.

Um die Gleichung numerisch lösen zu können, führt man zunächst die FeautrierVariablen $u$ und $v$ ein durch

$$
\begin{aligned}
u(z, \mu, \nu) & :=\frac{1}{2}[I(\tau, \mu, \nu)+I(\tau,-\mu, \nu)] \\
v(z, \mu, \nu) & :=\frac{1}{2}[I(\tau, \mu, \nu)-I(\tau,-\mu, \nu)]
\end{aligned}
$$

für $0 \leq \mu \leq 1$. Damit ergibt sich ein gekoppeltes Gleichungssystem für $u$ und $v$ :

$$
\begin{aligned}
\mu \frac{\partial v(\tau, \mu, \nu)}{\partial \tau} & =u(\tau, \mu, \nu)-S(\tau, \nu), \\
\mu \frac{\partial u(\tau, \mu, \nu)}{\partial \tau} & =v(\tau, \mu, \nu) .
\end{aligned}
$$

Einsetzen von (2.33) in (2.32) bringt die Strahlungstransportgleichung auf ihre entkoppelte Form als Differentialgleichung 2. Ordnung:

$$
\mu^{2} \frac{\partial^{2} u(\tau, \mu, \nu)}{\partial \tau^{2}}=u(\tau, \mu, \nu)-S(\tau, \nu) .
$$

In dieser Form wird die Gleichung nun diskretisiert und numerisch gelöst. Im Code werden das Verfahren von Feautrier (1964) und die darauf basierende wesentlich effizientere Methode von Rybicki (1971) benutzt $t^{5}$.

Die Größen $J$ und $H$ können nun durch $u$ und $v$ ausgedrückt werden:

$$
\begin{aligned}
J(\tau, \nu) & =\frac{1}{2} \int_{-1}^{1} I(\tau, \mu, \nu) d \mu=\int_{0}^{1} u(\tau, \mu, \nu) d \mu \\
H(\tau, \nu) & =\frac{1}{2} \int_{-1}^{1} I(\tau, \mu, \nu) \mu d \mu=\int_{0}^{1} v(\tau, \mu, \nu) \mu d \mu .
\end{aligned}
$$

Die mittlere Intensität $J$ kann also vollständig aus der Größe $u$ berechnet werden, die als Lösung von (2.34) bekannt ist. Für die Berechnung des Flusses $H$ wird jedoch die Größe $v$ benötigt oder alternativ die Größe $K$ aus der Momentengleichung (2.45). Ist man nur am Fluss $H(0, \nu)$ der äußersten Schicht interessiert, so kann dieser noch ohne weitere Lösung der Gleichung (2.33) bestimmt werden. An der äußersten Schicht $\tau=0$ gilt für isolierte Sterne $u(0, \mu, \nu)=v(0, \mu, \nu)$. Für bestrahlte Sterne gilt dies nicht mehr, jedoch gilt hier aufgrund der Relationen (2.30) der Zusammenhang

$$
v(0, \mu, \nu)=u(0, \mu, \nu)-I(0,-\mu, \nu) .
$$

Da $I(0,-\mu, \nu)$ die einfallende Strahlung spezifiziert und somit bekannt ist, kann auch hier $H(0, \nu)$ ohne weiteres berechnet werden. Für die Berechnung von $H(\tau, \nu)$

\footnotetext{
${ }^{5}$ Andere Lösungsverfahren werden z.B. in Hubeny (2003) angesprochen.
} 
für andere Schichten ist jedoch entweder die Lösung von (2.33) notwendig oder alternativ dazu die Lösung der Momentengleichung (2.45).

Soll weiterhin der vollständige Intensitätsverlauf $I(\tau, \mu, \nu)$ bestimmt werden, so muss aufgrund von

$$
\begin{gathered}
I(\tau, \mu, \nu)=u(\tau, \mu, \nu)+v(\tau, \mu, \nu) \\
I(\tau,-\mu, \nu)=u(\tau, \mu, \nu)-v(\tau, \mu, \nu)
\end{gathered} \quad, 0 \leq \mu \leq 1
$$

unumgänglich (2.33) zusätzlich gelöst werden.

Randbedingungen der Strahlungstransportgleichung Zur eindeutigen Bestimmung der Lösung der Strahlungstransportgleichung ist die Spezifikation der Randbedingungen notwendig. Dazu müssen zwei Funktionen

$$
\begin{aligned}
& I^{+}(\mu, \nu):=I\left(\tau_{\max }, \mu, \nu\right) \\
& I^{-}(\mu, \nu):=I(0,-\mu, \nu)
\end{aligned}
$$

für $\mu \geq 0$ angegeben werden. Das heißt, es wird am unteren Rand der Atmosphäre die ausgehende Strahlung $I^{+}$und am oberen Rand die einfallende Strahlung $I^{-}$ definiert.

Für die Spezifikation der Randbedingungen bei $\tau=\tau_{\max }$, am unteren „Rand“ der Atmosphäre, wird angenommen, dass hier die Diffusionsnäherung gültig ist. Diese Näherung basiert darauf, dass in großen Tiefen die Strahlung in nullter Näherung isotrop wird, so dass $I\left(\tau_{\max }, \mu, \nu\right) \approx S\left(\tau_{\max }, \nu\right)$ und somit $J\left(\tau_{\max }, \nu\right) \approx S\left(\tau_{\max }, \nu\right)$. Durch den Ausdruck (2.26) für die Ergiebigkeit folgt daraus $S\left(\tau_{\max }, \nu\right) \approx B\left(\tau_{\max }, \nu\right)$. Durch Benutzung der Strahlungstransportgleichung findet man mit diesen Werten einen Ausdruck in erster Näherung für die Intensität

$$
I^{+}(\mu, \nu) \approx B\left(\tau_{\max }, \nu\right)+\left.\mu \frac{d B(\tau, \nu)}{d \tau}\right|_{\tau=\tau_{\max }} .
$$

In der Tiefe $\tau=0$, am äußeren Rand der Atmosphäre, wird die vorgegebene einfallende Strahlung $I_{\text {irr }}(\mu, \nu)$ spezifiziert:

$$
I^{-}(\mu, \nu)=I(0,-\mu, \nu)=I_{\operatorname{irr}}(\mu, \nu), \quad 0 \leq \mu \leq 1 .
$$

Für Atmosphären isolierter Sterne ist

$$
I_{\text {irr }}(\mu, \nu)=0 .
$$

Für bestrahlte Atmosphären wird die Intensität der einfallenden Strahlung, die auch mit dem Winkel $\mu$ variieren kann, durch $I_{\text {irr }}(\mu, \nu)$ beschrieben. 
Momentengleichungen Die Integration der Strahlungstransportgleichung über alle Richtungen liefert

$$
\frac{\partial H(\tau, \nu)}{\partial \tau}=J(\tau, \nu)-S(\tau, \nu)
$$

Weiterhin liefert die Multiplikation der Strahlungstransportgleichung mit $\mu$ mit nachfolgender Integration über alle Richtungen

$$
\frac{\partial K(\tau, \nu)}{\partial \tau}=H(\tau, \nu)
$$

Man bezeichnet diese Gleichungen auch als Momentengleichungen nullter bzw. erster Ordnung.

\subsubsection{Hydrostatisches Gleichgewicht}

Die Gleichgewichtsbedingung im hydrostatischen Fall, bei der ein äußeres homogenes Gravitationsfeld $\vec{g}$ wirkt, lautet

$$
\nabla p(\vec{r})=\rho(\vec{r}) \vec{g}
$$

In der vorliegenden Modellgeometrie reduziert sich das auf

$$
\frac{\partial p(z)}{\partial z}=\rho(z) g
$$

Dabei ist $p$ der Gesamtdruck, der sich aus dem Gasdruck $p_{\text {gas }}$ und dem Strahlungsdruck $p_{\text {rad }}$ zusammensetzt:

$$
p=p_{\text {gas }}+p_{\text {rad }} .
$$

\subsubsection{Strahlungsgleichgewicht und Temperaturkorrektur}

Die Lösung der Strahlungstransportgleichung garantiert noch nicht die Gültigkeit des Modells. Da davon ausgegangen wird, dass in der Atmosphärenschicht keine Energieerzeugung stattfindet, muss der Gesamtstrahlungsfluss in jeder Schicht erhalten sein. Ein Atmosphärenmodell ist genau dann konsistent, wenn der Temperaturverlauf eine Lösung der Strahlungstransportgleichung ergibt, die das Strahlungsgleichgewicht

$$
\int_{0}^{\infty} \chi(\tau, \nu)[J(\tau, \nu)-S(\tau, \nu)] d \nu=\int_{0}^{\infty} \kappa(\tau, \nu)[J(\tau, \nu)-B(\tau, \nu)] d \nu=0
$$

erfüllt, wobei beim zweiten Ausdruck die spezielle Form (2.26) der Ergiebigkeit $S$ benutzt wurde. In Zusammenhang mit der Momentengleichung (2.44) kann das auch durch die Gleichung

$$
\int_{0}^{\infty} \chi \frac{\partial H(\tau, \nu)}{\partial \tau} d \nu=0
$$


ausgedrückt werden. Eine Abweichung von dieser Bedingung erfordert eine Modifikation der Temperaturschichtung, die nach dem in Gehren (1975) beschriebenen Verfahren erfolgt:

Gesucht wird ein $\delta T(\tau)$, welches für die gegebene Temperaturverteilung $T(\tau)$ einen besseren Wert $T(\tau)+\delta T(\tau)$ liefert. Ausgangspunkt des Verfahrens sind die beiden Momentengleichungen (2.44) und (2.45), in der $H$ und $K$ ersetzt werden durch $J$ und die Eddington Faktoren

$$
\begin{aligned}
f_{H}(\tau, \nu) & :=\frac{H(\tau, \nu)}{J(\tau, \nu)} \\
f_{K}(\tau, \nu) & :=\frac{K(\tau, \nu)}{J(\tau, \nu)} .
\end{aligned}
$$

Die Momentengleichungen nehmen dann folgende Form an:

$$
\begin{aligned}
& \frac{\partial^{2}\left(f_{K} J\right)}{\partial \tau^{2}}=J-S \\
& \frac{\partial\left(f_{K} J\right)}{\partial \tau}=f_{H} J .
\end{aligned}
$$

Diese beiden Gleichungen werden simultan mit der Bedingung des Strahlungsgleichgewichtes (2.49) bzw. (2.50) behandelt. Um nun einen besseren Wert für die Temperatur zu erhalten wird, $B(\tau, \nu)$ ersetzt durch

$$
\tilde{B}(\tau, \nu) \equiv B(T(\tau)+\delta T(\tau), \nu) .
$$

Die lineare Approximation liefert

$$
\tilde{B}(\tau, \nu) \approx B(\tau, \nu)+\frac{d B(T(\tau), \nu)}{d T(\tau)} \delta T(\tau)
$$

Durch das simultane Lösen der Momentengleichung mit der Zwangsbedingung des Strahlungsgleichgewichts erhält man nun eine Lösung für $\delta T(\tau)$.

Es zeigt sich, dass bei bestrahlten Atmosphären eine Dämpfung der Lösung notwendig ist, um die Stabilität zu verbessern. Zu einer Temperaturverteilung $T_{i}(\tau)$ der Iteration $i$ wird eine Differenzverteilung $\delta T_{i}(\tau)$ berechnet, die zu einer neuen „besseren" Verteilung für die Iteration $i+1$ führt:

$$
T_{i+1}(\tau)=T_{i}(\tau)+\delta T_{i}(\tau)
$$

Die Korrektur $\delta T$ wird nun durch einen Schwellenwert begrenzt.

$$
\delta T(\tau)= \begin{cases}\delta T(\tau) & , \text { für }|\delta T(\tau)| \leq p T(\tau) \\ p T(\tau) & , \text { für }|\delta T(\tau)|>p T(\tau)\end{cases}
$$

Dabei wurden mit $p=0.1-0.2$ akzeptable Ergebnisse erzielt. Eine weitere Idee zu einem zusätzlichen Glättungsverfahren findet sich in Madej \& Różańska (2000). Es wird das geometrische Mittel aus zwei Temperaturschichtungen aufeinanderfolgender Iterationen gebildet:

$$
T_{i+1}(\tau)=\sqrt{T_{i}(\tau)\left[T_{i}(\tau)+\delta T(\tau)\right]} .
$$




\subsubsection{Iterative Lösungsstruktur}

Die Lösung des Atmosphärenmodells besteht aus den Schritten

1. Berechnung des Materiezustands auf Basis einer gegebenen Temperaturstruktur, Berechnung der Opazitäten

2. Lösung der hydrostatischen Gleichung: 2.1.4

3. Lösung der Strahlungstransportgleichung: 2.1 .3

4. Prüfen der Konsistenz des Modells (Strahlungsgleichgewicht)

5. Bei Verletzung von 4: Korrektur der Temperaturstruktur (2.1.5) und erneuter Start bei 1

Hierdurch ist eine iterative Struktur gegeben, die allmählich gegen die gesuchte Lösung des Modells konvergiert, wenn man voraussetzt, dass das Temperaturkorrekturverfahren tatsächlich eine bessere Lösung liefert.

\subsubsection{Weitere Details zur Modellberechnung}

Zur Interpretation des Spektrums Ein Atmosphärenmodell beinhaltet eine Lösung $F(\tau, \nu)$, die den Strahlungsfluss der Schicht $\tau$ beschreibt. Hauptsächlich ist man an der Strahlung interessiert, die den Beobachter erreicht, d.h. der Strahlung, die aus der äußersten Schicht entweicht. Den Fluss $F(\tau, \nu)$ kann man aufspalten in einen ausgehenden $(\mu>0)$ und einen einfallenden $(\mu<0)$ Anteil:

$$
\begin{aligned}
F(\tau, \nu) & =2 \pi \int_{-1}^{1} I(\tau, \mu, \nu) \mu d \mu \\
& =2 \pi \int_{-1}^{0} I(\tau, \mu, \nu) \mu d \mu+2 \pi \int_{0}^{1} I(\tau, \mu, \nu) \mu d \mu \\
& =F_{\text {in }}(\tau, \nu)+F_{\text {out }}(\tau, \nu) .
\end{aligned}
$$

Mit $F(\tau)$ bezeichnen wir den integrierten Gesamtfluss

$$
F(\tau):=\int_{0}^{\infty} F(\tau, \nu) d \nu
$$

Bei einem isolierten Stern ist $F_{\text {in }}(0, \nu)=0$. Somit kann das beobachtbare Spektrum unmittelbar mit dem Fluss $F(0, \nu)=F_{\text {out }}(0, \nu)$ identifiziert werden. Bei einem bestrahlten Stern gilt dies jedoch nicht mehr, da hier $F_{\text {in }}(0, \nu) \neq 0$ ist. Hier muss unterschieden werden zwischen dem Fluss $F(0, \nu)$ und dem beobachtbaren Spektrum, welches gegeben ist durch $F_{\text {out }}(0, \nu)$. 
Erhöhung der Bestrahlungsstärke Es ist das Ziel, die Atmosphäre mit sehr hohen Strahlungsflüssen $F_{\text {in }}$ zu bestrahlen, die über ein Vielfaches des intrinsischen Flusses $F_{\mathrm{wd}}=\sigma_{\mathrm{sb}} T_{\text {eff }}^{4}$ des Weißen Zwerges, der mit der Effektivtemperatur $T_{\text {eff }}$ über die Stefan-Boltzmann-Kostante $\sigma_{\mathrm{sb}}=5.6705 \cdot 10^{-5} \mathrm{erg} \mathrm{cm}^{-2} \mathrm{~s}^{-1} \mathrm{~K}^{-4}$ verknüpft ist, hinausgehen. Hierbei zeigt sich, dass die direkte Berechnung - ausgehend von einer ungestörten Atmosphäre - zu numerischen Komplikationen führt, so dass die Temperaturkorrektur keine Konvergenz der Modelle liefert. Hier werden bessere Resultate erzielt, wenn die Erhöhung des Bestrahlungsflusses schrittweise erfolgt.

Das Modell $\mathcal{M}$ mit der Effektivtemperatur $T_{\text {eff }}$, der Oberflächenschwerebeschleunigung $g$ und der Einstrahlung $I_{\text {irr }}(\mu, \nu)$, die den Bestrahlungsfluss $F_{\text {in }}$ ergibt, liefert bei erfolgreicher Konvergenz eine Temperaturverteilung $T[\mathcal{M}](\tau)$. Zu Beginn des Iterationsprozesses muss eine Ausgangstemperaturverteilung $T_{0}(\tau)$ angenommen werden. Diese ist im Normalfall die einer grauen Atmosphäre ohne Bestrahlung. Eine graue Atmosphäre ist dadurch definiert, dass die Opazität $\chi$ nicht von der Frequenz $\nu$ abhängt. Die Temperaturverteilung $T_{\text {grey }}(\tau)$ kann dann berechnet und durch einen geschlossenen Ausdruck angegeben werden (Mihalas, 1978). Für die Temperaturschichtung zu Beginn des Iterationsprozesses in at gilt dann:

$$
T_{0}(\tau)=T_{\text {grey }}(\tau)
$$

Bei einem zu großen Bestrahlungsfluß $F_{\text {in }}$ führt die Temperaturkorrektur mit der Starttemperaturverteilung aus (2.62) nicht mehr zu einer Konvergenz des Modells. Bei der schrittweisen Erhöhung des Bestrahlungsflusses wird als Starttemperaturverteilung $T_{0}(\tau)$ nicht (2.62) verwendet, sondern die Temperaturverteilung des letzten berechneten Modells. Bezeichnet man die berechnete Temperaturverteilung des Modells $\mathcal{M}\left(F_{\text {in }}\right)$ mit $T\left[\mathcal{M}\left(F_{\text {in }}\right)\right](\tau)$ und die Starttemperaturverteilung des neuen Modells $\mathcal{M}\left(F_{\text {in }}+\delta F\right)$ mit $T_{0}\left[\mathcal{M}\left(F_{\text {in }}+\delta F\right)\right]$, so setzen wir

$$
T_{0}\left[\mathcal{M}\left(F_{\text {in }}+\delta F\right)\right](\tau)=T\left[\mathcal{M}\left(F_{\text {in }}\right)\right](\tau) .
$$

\section{$2.2 \quad$ Ergebnisse}

\subsubsection{Isotrope Bestrahlung mit Schwarzkörperstrahlung}

Wir betrachten zunächst isotrope Bestrahlung der Atmosphäre. Die Ergebnisse dieses Teils der Arbeit wurden in König \& Gänsicke (2003) veröffentlicht. Die Intensität der Bestrahlung ist gegeben durch ein Schwarzkörperspektrum

$$
B(\nu, T)=\frac{2 h}{c^{2}} \frac{\nu^{3}}{e^{\frac{h \nu}{k T}}-1} .
$$

Diese Form des Spektrums wird dadurch motiviert, dass Zyklotronstrahlung durch ein Schwarzkörperspektrum approximiert werden kann. Dabei ist hier nur die Form 
des Spektrums von Interesse, der Gesamtstrahlungsfluss $F_{\text {int }}$, der für einen Schwarzkörper durch das Stefan-Boltzmann Gesetz

$$
F_{\mathrm{in}}=\int_{0}^{\infty} \pi B\left(\nu, T_{\mathrm{bb}}\right) d \nu=\sigma_{\mathrm{sb}} T_{\mathrm{bb}}^{4}
$$

gegeben ist, ist dabei nicht bindend für die Modellbestrahlungsstärke. Vielmehr sollen diese beide Parameter voneinander entkoppelt sein. Dies wird dadurch erreicht, dass die Bestrahlungsintensität mit einem zusätzlichen Parameter skaliert wird.

Die Bestrahlung wird definiert durch

$$
I^{-}(\mu, \nu)=x \cdot\left(\frac{T_{\mathrm{eff}}}{T_{\mathrm{bb}}}\right)^{4} B\left(\nu, T_{\mathrm{bb}}\right),
$$

wobei $\left(x, T_{\mathrm{bb}}, T_{\text {eff }}\right)$ Parameter des Modells sind. Es können also Form der spektralen Verteilung und Gesamtbestrahlungsfluss unabhängig voneinander variiert werden. Das ist zum einen notwendig, da höhere Bestrahlungsflüsse aus technischen Gründen nur schrittweise berechnet werden können. Zum anderen interessiert hier hauptsächlich der Schwarzkörper als Modell für Zyklotronemission, dabei handelt es sich um nicht-thermische Strahlung. Die Approximation der Zyklotronstrahlung durch Schwarzkörper wurde in Abschnitt 1.2 dargestellt. Diese Form der Bestrahlung ist unabhängig vom Winkel $\mu$. Der Sinn der Normierung mit dem Faktor $\left(T_{\text {eff }} / T_{\mathrm{bb}}\right)^{4}$ wird offensichtlich, wenn man den Gesamtbestrahlungsfluss berechnet:

$$
F_{\mathrm{in}}=x \cdot \sigma_{\mathrm{sb}} \cdot T_{\mathrm{eff}}^{4}=x \cdot F_{\mathrm{wd}}, \quad \text { bzw. } x=\frac{F_{\mathrm{in}}}{F_{\mathrm{wd}}} .
$$

Die vollständige Spezifikation des Modells erfordert noch die Angabe der Effektivtemperatur $T_{\text {eff }}$ und der Oberflächenschwerebeschleunigung $g$. Wir benutzen folgende Werte

$$
T_{\text {eff }}=20000 \mathrm{~K}, \quad \log \left[\mathrm{g} /\left(\mathrm{cm} \mathrm{s}^{-2}\right)\right]=8 .
$$

Der Fluss des Sterns beträgt demnach

$$
F_{\mathrm{wd}}=9.073 \cdot 10^{12} \mathrm{erg} \mathrm{s}^{-1} \mathrm{~cm}^{-2} .
$$

Bestrahlung mit $B(\nu, T=10000 \mathrm{~K})$ Betrachten wir zunächst ein Modell, bei dem die Bestrahlung die Form eines Schwarzkörperspektrums mit der Temperatur $T=10000 \mathrm{~K}$ besitzt. Es wurden Bestrahlungsflüsse $F_{\text {in }}$ im Bereich von

$$
x=\frac{F_{\text {in }}}{F_{\mathrm{wd}}}=(0.1-65) F_{\mathrm{wd}}
$$

angenommen. Die obere Grenze ist dadurch gegeben, dass die Lösung der Atmosphärenstruktur bei größerer Bestrahlung zunehmend instabil wird. 




Abbildung 2.2: Strahlungsflüsse einer Modellatmosphäre, die mit einem integrierten Bestrahlungsfluss von $F_{\text {in }}=4.5 F_{\text {wd }}$ bestrahlt wird. Die durchgezogene Kurve repräsentiert den ausgehenden Fluss $F_{\text {out }}(\lambda)$, die kurz gestrichelte den Bestrahlungsfluss $F_{\text {in }}(\lambda)$ und die lang gestrichelte den Gesamtfluss $F(\lambda)=F_{\text {in }}(\lambda)+$ $F_{\text {out }}(\lambda)$. Die gepunktete Kurve stellt zum Vergleich das Spektrum $F_{\text {wd }}(\lambda)$ eines unbestrahlten Weißen Zwerges dar. Für die integrierten Flüsse gilt die Relation $\int F(\lambda) d \lambda=\int F_{\text {in }}(\lambda) d \lambda+\int F_{\text {out }}(\lambda) d \lambda=\int F_{\text {wd }}(\lambda) d \lambda=\sigma T_{\text {eff }}^{4}$.

In Abbildung 2.2 sehen wir verschiedene Komponenten des Strahlungsflusses der äußersten Schicht der Atmosphäre für einen repräsentativen Bestrahlungfluss $F_{\text {in }}=4.5 F_{\text {wd }}$. Die gepunktete Kurve stellt zum Vergleich das Spektrum $F_{\mathrm{wd}}(\lambda)$ eines unbestrahlten Weißen Zwerges mit einer Effektivtemperatur von $T_{\text {eff }}=20000 \mathrm{~K}$ dar. Der Bestrahlungsfluss $F_{\text {in }}(\lambda)$ ist kurz gestrichelt gezeichnet. Der ausgehende Fluss $F_{\text {out }}(\lambda)$ ist durch die durchgezogene Kurve dargestellt, der als für den Beobachter sichtbares Spektrum zu interpretieren ist. Der Gesamtfluss $F(\lambda)$ ist lang gestrichelt gezeichnet. Für diesen gilt $F(\lambda)=F_{\text {in }}(\lambda)+F_{\text {out }}(\lambda)$, bzw. für den integrierten Fluss

$$
F=F_{\text {in }}+F_{\text {out }}=F_{\mathrm{wd}}=\sigma_{\mathrm{sb}} T_{\text {eff }} .
$$

Man kann hier erkennen, wie der Fluss der Atmosphäre auf die Bestrahlung reagiert. Das Maximum des Bestrahlungsflusses liegt bei $\log (\lambda / \AA) \approx 3.5$. In dieser Region 


\begin{tabular}{cc}
\hline$F_{\text {in }} / F_{\text {wd }}$ & $F_{\text {in }} /\left(10^{12} \mathrm{erg} \mathrm{s}^{-1} \mathrm{~cm}^{-2}\right)$ \\
\hline 1.7 & 15.9 \\
3.4 & 30.9 \\
6.6 & 60.3 \\
17.2 & 156.3 \\
30.5 & 277.0 \\
\hline
\end{tabular}

Tabelle 2.1: Gesamtbestrahlungsflüsse für die Modellrechnungen in Abb. 2.3

wird der Gesamtfluss $F(\lambda)$ sogar negativ, was nichts anderes bedeutet, als dass mehr Strahlung in die Atmosphäre eindringt als die Atmosphäre abstrahlt. Um jedoch das Strahlungsgleichgewicht zu erhalten, muss die Atmosphäre diese Strahlung in einem anderen Wellenlängenbereich wieder abstrahlen. Dies geschieht durch eine Temperaturerhöhung, die zu einer Vergrößerung des thermischen Anteils in der Ergiebigkeit $S$ führt. Da eine Temperaturerhöhung das Maximun der Schwarzkörperfunktion in den Bereich höherer Frequenzen verschiebt, liegt das Maximum des Flusses im Diagramm nun im Bereich kleinerer Wellenlängen. Dass der Fluss $F$ in gewissen Frequenzbereichen negativ ist, stellt keinerlei Problem dar, da zur Erhaltung des Strahlungsgleichgewichts lediglich der integrale Gesamtfluss

$$
F=\int_{0}^{\infty} F(\nu) d \nu
$$

relevant ist.

Neben einer Zunahme des Kontinuumsflusses, der den Bestrahlungsfluss kompensiert, verändert sich auch die Struktur der Spektrallinien. Mit zunehmendem Bestrahlungsfluss wird die Absorption in den Linien geringer, bis letztendlich der Linienkern in Emission „umkippt“. Dies hängt mit der Modifikation der Temperaturschichtung zusammen, die durch die Bestrahlung verursacht wird.

Abbildung 2.3 zeigt eine Darstellung des ausgehenden Flusses $F_{\text {out }}$ in der Lyman-Region für verschiedene Bestrahlungsflüsse aus Tabelle 2.1. Darunter befinden sich die entsprechenden Temperaturverteilungen der Atmosphärenmodelle, wobei bei der Dastellung die Rosselandsche Tiefe benutzt wurde. Sie ist definiert durch $d \tau_{\text {ross }}=-\chi_{\text {ross }} d z$, mit

$$
\chi_{\mathrm{ross}}^{-1}=\frac{\int \chi(\nu)^{-1} \frac{\partial B(\nu, T)}{\partial T} d \nu}{\int \frac{\partial B(\nu, T)}{\partial T} d \nu} .
$$

Die Bestrahlung führt zunächst zu einer Erhöhung der Temperatur um einen bis zu einer gewissen Grenztiefe $\tau_{\mathrm{c}}$ (fast) konstanten Anteil. Ab der Tiefe $\tau_{\mathrm{c}}$ mündet die Verteilung wieder in die der ungestörten Atmosphärenstruktur. Diese Form der Temperaturverteilung wurde in Gänsicke et al. (1998) a priori angenommen, um zu versuchen, die beobachteten fehlenden Absorptionsfeatures in den HST/GHRS-Spektren 

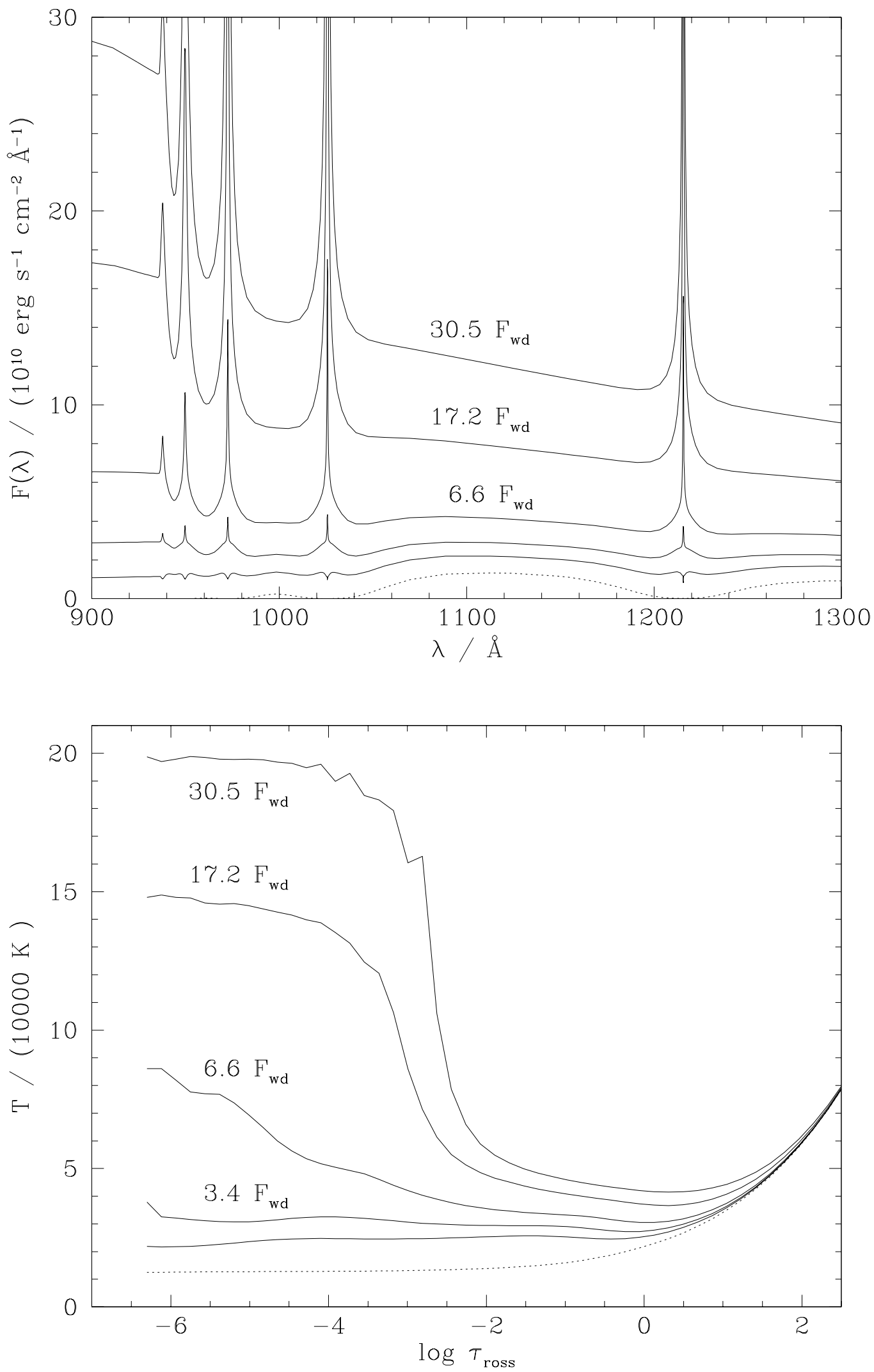

Abbildung 2.3: Oben: Ausgehender Fluss $F_{\text {out }}(\lambda)$ in der Lyman-Region $1150 \AA \leq \lambda \leq 1400 \AA$. Unten: Temperaturstruktur der Atmosphäre. Die einzelnen Kurven beziehen sich auf die Bestrahlungsflüsse $F_{\text {in }}=x F_{\text {wd }}$ mit $x=1.7,3.4,6.6,17.2,30.5$ (von unten nach oben). Die gepunktete Kurve repräsentiert die Temperaturschichtung eines unbestrahlten Weißen Zwerges. 
von AM Her zu erklären. Die Rechnungen zeigen, dass eine solche annähernd isotherme Struktur für einen gewissen Bestrahlungsbereich in der Tat von einem konsistenten Atmosphärenmodell geliefert wird.

Weiterhin zeigt sich allerdings mit zunehmendem Bestrahlungfluss eine Inversion der Temperaturstruktur. Damit ist gemeint, dass die Temperatur in den äußeren Schichten höher ist, nach innen abfällt, um dann wieder ab einer Grenztiefe $\tau_{\mathrm{c}}$ in die normale ungestörte Atmosphärenstruktur überzugehen. Die zunehmende Inversion der Temperaturstruktur verursacht die Ausbildung von Emissionslinien im Spektrum, wie man im oberen Diagramm der Abbildung 2.3 erkennen kann.

Wenn man die Intensität $I(z)$ für eine Schicht bei $z$ kennt und die Intensität $I(z+\Delta z)$ für die Schicht $z+\Delta z$ nähert, bekommt man

$$
I(z+\Delta z) \approx I(z)+\frac{\partial I}{\partial z} \Delta z
$$

Setzt man diesen Ausdruck in die Strahlungstransportgleichung (2.28) ein, wobei näherungsweise $S=B$ gesetzt wird, ergibt das

$$
I(z+\Delta z) \approx I(z)+\frac{\chi}{\mu} \underbrace{[B(T)-I(z)] \Delta z}_{=K(T)} .
$$

Die Intensität an der neuen Stelle $z+\Delta z$ ist folglich die Intensität an der alten Stelle $z$ plus einem Term $\chi / \mu K(T)$. Ist die von der Strahlung durchdrungene Materie sehr „heiß“, so ist

$$
B(T)>I(z) \Rightarrow K(T)>0
$$

Es kommt zur Emission, da das Linienprofil, welches durch $\chi(\nu)$ gegeben ist, zur ursprünglichen Intensität hinzuaddiert wird. Ist im Gegensatz dazu die Materie „kalt“, so gilt

$$
B(T)<I(z) \Rightarrow K(T)<0
$$

was zur Absorption führt.

Die schmalen Emissionskerne entstehen in einer Tiefenregion von $\tau_{\text {ross }} \approx 10^{-3}$ bis zum Rand. Das Profil dieser Kerne wird hauptsächlich bestimmt durch die Dopplerverbeiterung. Da der optisch dünne Entstehungsbereich stark geheizt wird, könnten hier Effekte durch Abweichungen vom lokalen thermodynamischen Gleichgewicht auftreten, die hier nicht berücksichtigt wird. Der breite Anteil der Linien entsteht in tieferen Schichten ab $\tau_{\text {ross }} \approx 10^{-1}$. Dort wird das Linienprofil dominiert von der Druckverbreiterung. Diese Teile des Profils werden auch im LTE korrekt beschrieben. 


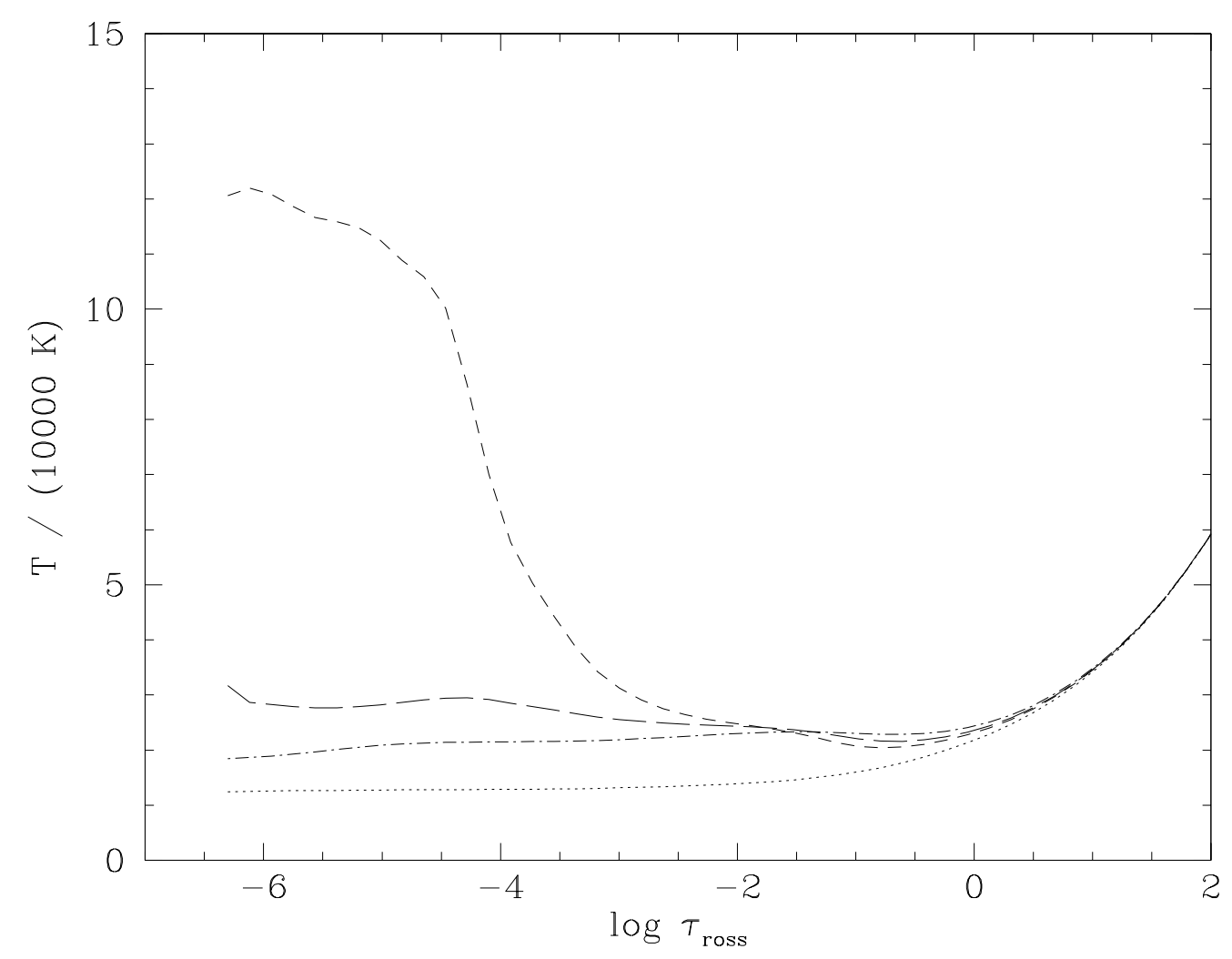

Abbildung 2.4: Vergleich der Temperaturverteilungen bei verschiedenen Bestrahlungstemperaturen $T_{\mathrm{bb}}$ und gleichen $F_{\mathrm{in}}=1.1 F_{\mathrm{wd}}$. Die kurz gestrichelten, lang gestrichelten und gepunktet gestrichelten Kurven repräsentieren die Verteilungen mit den Bestrahlungstemperaturen $T_{\mathrm{bb}}=3000,5000,10000 \mathrm{~K}$. Die gepunktete Kurve repräsentiert die Temperaturverteilung eines unbestrahlten Weißen Zwerges.

Grenzen der Modellrechnungen Bei den Berechnungen der Atmosphärenmodelle zeigt sich, dass es obere Grenzen für den Bestrahlungsfluss $F_{\text {in }}$ gibt. Diese Grenze hängt stark davon ab, in welchem Wellenlängenbereich die Einstrahlung stattfindet. Bei Rechnungen mit verschiedenen Bestrahlungsformen durch verschiedene Schwarzkörpertemperaturen $T$ zeigt sich die Tendenz, dass die maximal mögliche Bestrahlung mit zunehmender Temperatur (bei gleicher Skalierung des Gesamtflusses) zunächst zunimmt und nach einer Temperatur von etwa $T=10000 \mathrm{~K}$ sehr stark abnimmt. Vergleichsrechnungen zeigten die Grenzen aus Tabelle 2.2. Es fällt auf, dass die maximal mögliche Einstrahlung bei Temperaturen $T_{\mathrm{bb}}>10000 \mathrm{~K}$ sehr stark abnimmt. Dies hängt damit zusammen, dass bei diesen Temperaturen ein signifikanter Anteil der Bestrahlung in den Bereich der Ly $\alpha$-Linie gelangt. Weiterhin zeigt sich aber auch, dass bei niedrigeren Temperaturen $T_{\mathrm{bb}} \leq 5000$ der Wert leicht abnimmt. Hier zeigt sich, dass die Bestrahlung mit niedriger Temperatur schneller in den äußeren Schichten absorbiert wird und somit schneller zu einer Temperatur- 


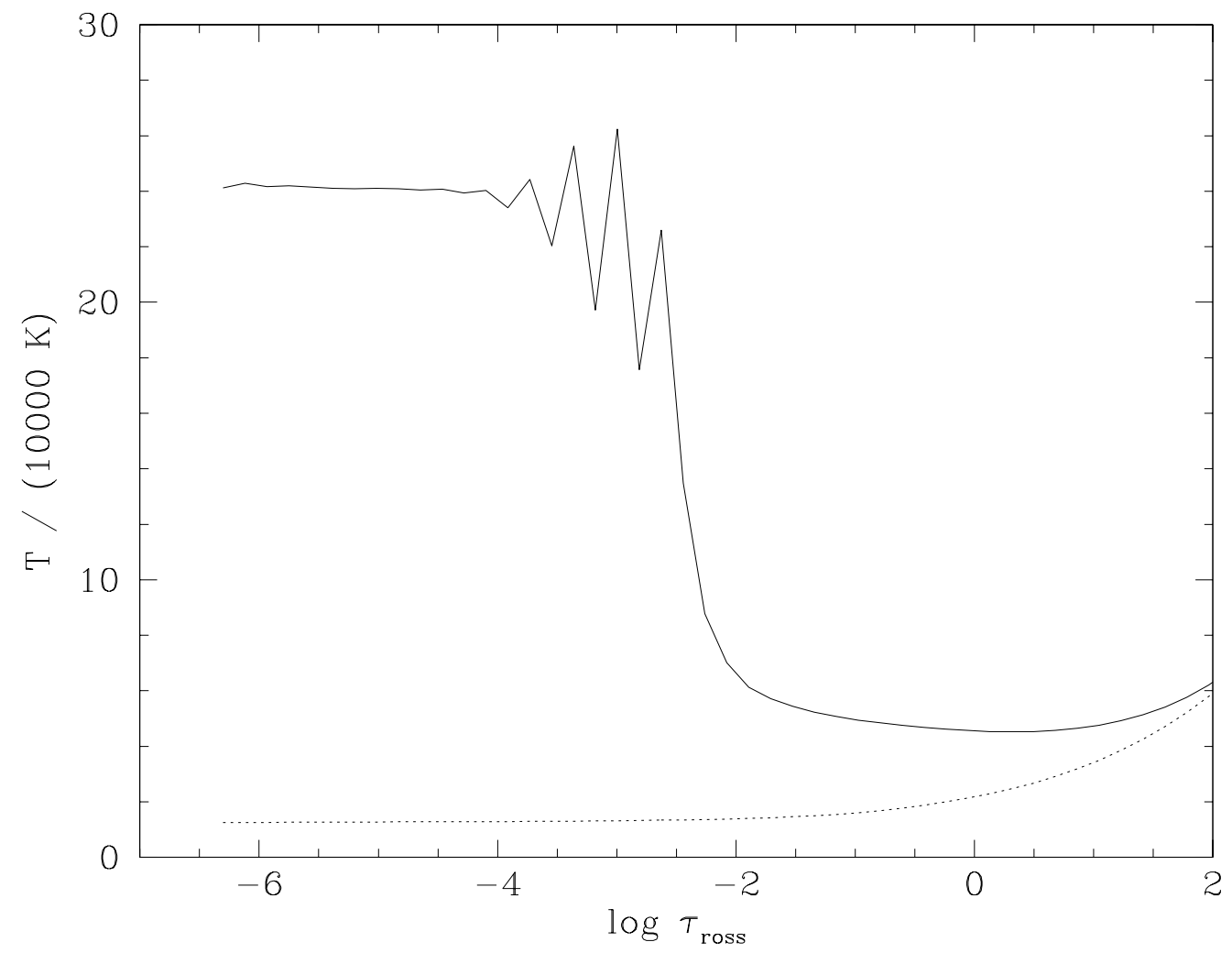

Abbildung 2.5: Temperaturschichtung einer Modellatmosphäre die mit einem Fluss von $F_{\text {in }}=45 F_{\text {wd }}$ bestrahlt wird. Die Temperatur beträgt $T_{\mathrm{bb}}=10000 \mathrm{~K}$. Das Modell konvergiert noch, obwohl die Struktur offensichtlich nicht physikalisch ist. Zum Vergleich ist die Temperaturschichtung eines unbestrahlten Modells gepunktet gezeichnet.

Tabelle 2.2: Maximale Bestrahlungsflüsse $F_{\text {in }}$, bei denen die Lösung der Atmosphärenstruktur instabil wird, in Abhängigkeit von der Schwarzkörpertemperatur $T_{\mathrm{bb}}$ der einfallenden Strahlung.

\begin{tabular}{ccccccc}
\hline$T_{\mathrm{bb}} / \mathrm{K}$ & 3000 & 5000 & 10000 & 15000 & 20000 & 25000 \\
\hline$F_{\text {in }} / F_{\mathrm{wd}}$ & 2 & 12 & 65 & 0.3 & 0.2 & $<0.1$ \\
\hline
\end{tabular}

inversion führt.

Es stellt sich noch die Frage, ob die festgestellten Grenzen numerischer Natur sind oder eine physikalische Grundlage besitzen. Es ist zu vermuten, dass die Atmosphäre physikalisch instabil wird, wenn sie in den äußeren Schichten Temperaturen animmt, die dazu führen, dass dort ein Großteil der Materie der das 
Tabelle 2.3: Das Winkelgitter der Modellrechnungen mit winkelabhängiger Einstrahlung. $\mu=0.1$ entspricht der flachsten Einstrahlung, $\mu=1$ senkrechter Bestrahlung.

\begin{tabular}{ccccccccccc}
\hline$\mu$ & 0.1 & 0.2 & 0.3 & 0.4 & 0.5 & 0.6 & 0.7 & 0.8 & 0.9 & 1.0 \\
\hline$\theta$ & 84.3 & 78.5 & 72.5 & 66.4 & 60.0 & 53.1 & 45.6 & 36.9 & 25.8 & 0 \\
\hline
\end{tabular}

Gravitationsfeld des Sterns überwinden kann. Die mittlere kinetische Energie beträgt $E=3 / 2 k T=1 / 2 m \bar{v}^{2}$. Bei einer Maximaltemperatur von $T=300000 \mathrm{~K}$ in der äußersten Schicht beträgt $\bar{v}=86 \mathrm{~km} \mathrm{~s}^{-1}$. Die Fluchtgeschwindigkeit hingegen ist $v_{\text {esc }}=\sqrt{2 G M / R}=3990 \mathrm{~km} \mathrm{~s}^{-1}$, mit den Parametern $M=0.6 \mathrm{M}_{\odot}$ und $R=10^{9} \mathrm{~cm}$. Die mittlere Geschwindigkeit liegt nach dieser Abschätzung weit unterhalb der Entweichgeschwindigkeit. Eine weitere physikalische Grenze liefert die Eddington-Leuchtkraft $L_{\text {Edd }}$, die sich dadurch ergibt, dass die Beschleunigung durch das Strahlungsfeld $g_{\text {rad }} \approx \sigma_{\mathrm{T}} / c F$ kleiner sein muss als die Schwerebeschleunigung $g=G M / R^{2}$. Dies führt hier zu der Bedingung $F \lesssim 6 \cdot 10^{18} \mathrm{erg} \mathrm{s}^{-1} \mathrm{~cm}^{-2}$, von der der erreichte maximale ausgehende Bestrahlungsfluss mit $F_{\text {out }} \approx 6 \cdot 10^{14} \mathrm{erg} \mathrm{s}^{-1} \mathrm{~cm}^{-2}$ weit entfernt ist. Auch wenn es sich bei diesen Betrachtungen um grobe Näherungen handelt, ist $\mathrm{zu}$ vermuten, dass eine physikalische Grenze noch nicht erreicht wird. Dafür spricht auch, dass die Instabilitäten bei den Bestrahlungstemperaturen $T_{\mathrm{bb}}>10000 \mathrm{~K}$ schon auftreten, bevor eine signifikante Erhitzung der Atmosphäre eintritt. Hier kommt es vor, dass die Temperaturkorrektur teilweise in die falsche Richtung korrigiert. Für kleinere Bestrahlungstemperaturen $T<=10000 \mathrm{~K}$ lässt sich feststellen, dass mit zunehmender Einstrahlung die Anzahl der zur Konvergenz notwendigen Iterationsschritte zunimmt, da die Temperaturkorrektur anfängt, mit zunehmender Amplitude um den Lösungswert der Temperatur zu schwingen. Im Falle der hohen Bestrahlungsflüsse ist es nicht mehr möglich gewesen, diese Schwingungen zu dämpfen. Desweiteren mußte der Bestrahlungsfluss wie schon erwähnt schrittweise erhöht werden. Es bilden sich jedoch schon vor dem Versagen der Korrektur Lösungen der Temperaturstruktur aus, die offensichtlich nicht mehr physikalisch sind (Abbildung 2.5). Im gänzlich unbestrahlten Fall zeigte das Korrekturverfahren hingegen ein sehr gutes Konvergenzverhalten. Selbst bei bewusst exotisch gewählten Anfangstemperaturschichtungen, wurde nach einer relativ geringen Anzahl von Iterationen $\left(N_{\text {iter }}<10\right)$ die Lösung erreicht.

\subsubsection{Winkelabhängige Bestrahlung}

Im letzten Abschnitt war die Intensität der Einstrahlung unabhängig vom Winkel. Nun soll Bestrahlung betrachtet werden, die eine winkelabhängige Intensität besitzt, um die Abhängigkeit des Atmosphärenmodells vom Bestrahlungswinkel zu analysieren. 
Statt der Form (2.66) besitzt die Intensität der Einstrahlung nun die Form

$$
I^{-}(\mu, \nu)=x \cdot I_{0}(\nu) \cdot \delta\left(\mu-\mu_{0}\right),
$$

wobei die $\mu$ einem diskreten Winkelgitter entnommen sind und $\mu_{0}$ den Bestrahlungswinkel repräsentiert. $\delta$ entspricht der diskreten Delta-Funktion. Die Strahlung trifft lediglich unter einem bestimmten Bestrahlungswinkel $\mu_{0}$ auf die Atmosphäre. Dabei bestimmt $I_{0}(\nu)$ wiederum die Form des Bestrahlungsspektrums und ist hier gegeben durch die Planckfunktion

$$
I_{0}(\nu)=B\left(\nu, T_{\mathrm{bb}}\right)
$$

wobei hier eine Temperatur von $T_{\mathrm{bb}}=10000 \mathrm{~K}$ benutzt wird. Die Modellrechnungen fanden mit einem in $\mu$ äquidistanten Winkelgitter mit den 10 Winkeln aus Tabelle 2.3 statt. $\mu=1$ entspricht senkrechter Bestrahlung, die mit abnehmenden $\mu$ flacher wird. $\mu=0.1$ enspricht der flachsten betrachteten Einstrahlung.

Abbildung 2.7 zeigt die winkelabhängigen Intensitäten verschiedener Modelle mit unterschiedlichen Bestrahlungswinkeln unter verschiedenen Sichtwinkeln. In jedem Diagramm sind zwei Kurvenpaare zu sehen, die zu den Bestrahlungsflüssen $F_{\text {in }}=1.0 F_{\text {wd }}$ und $F_{\text {in }}=6.7 F_{\text {wd }}$ gehören. Ein Kurvenpaar ist jeweils den Bestrahlungswinkeln $\mu=1.0$ (durchgezogene Kurve, senkrechte Bestrahlung) und $\mu=0.1$ (gestrichelte Kurve, flache Bestrahlung) zugeordnet. Die Abbildung oben zeigt die Intensitäten unter einem flachen Sichtwinkel von $\theta=84^{\circ}$. Die flache Bestrahlung (gestrichelt) zeigt Absorption in den Linienflügeln und Emission in den Linienkernen für beide Bestrahlungsflüsse. Die senkrechte Bestrahlung (durchgezogen) zeigt für den geringen Bestrahlungsfluss noch keine Emission in den Linienkernen. Für den höheren Bestrahlungsfluss bildet sich Emission aus, die im Vergleich zur senkrechten Bestrahlung jedoch geringer ausfällt. Weiterhin ist die Absorption in den Linienflügeln geringer ausgeprägt. Die untere Abbildung enspricht einem senkrechten Sichtwinkel von $\theta=0^{\circ}$. Hier zeigt sich generell, dass die Absorption in den Flügeln fast vollständig verschwunden ist. Lediglich die senkrechte Bestrahlung zeigt unter dem flachen Bestrahlungswinkel (untere durchgezogene Linie) ein wenig Absorption in den Linienkernen. In allen Fällen ist oberhalb der Lymankante im Kontinuum die Intensität der senkrechten Bestrahlung größer als die der flachen, während sich darunter die Verhältnisse umkehren.

Abbildung 2.6 zeigt einen Vergleich der Temperaturschichtungen $T(\tau)$ für zwei verschiedene Bestrahlungsflüsse $F_{\text {in }}=1.0 F_{\text {wd }}$ und $F_{\text {in }}=6.7 F_{\text {wd }}$. Zu jedem Bestrahlungsfluss sind zwei Kurven dargestellt. Die durchgezogene Kurve zeigt die Schichtung bei senkrechter Einstrahlung $(\mu=1)$, die gestrichelte flache Einstrahlung $(\mu=0.1)$. Es ist zu sehen, dass bei gleichem Bestrahlungsfluss $F_{\text {in }}$ die senkrechte Bestrahlung die tieferen Schichten der Atmosphäre besser heizt. Die flache Einstrahlung wird schon in den äußeren Schichten absorbiert.

Es wurde ein Gitter von Modellatmosphären gerechnet, das alle Bestrahlungswinkel aus Tabelle 2.3 abdeckt. Die Bestrahlungsflüsse erstrecken sich für $\mu=1$ 
Tabelle 2.4: Bestrahlungsflüsse $F_{\text {in }}$ für den Winkel $\mu=1$ der in den Abbildungen 2.8 bis 2.12 dargestellten Modellspektren. Der Bestrahlungsfluss für andere Winkel ergibt sich durch $F_{\text {in }}(\mu)=\mu \cdot F_{\text {in }}(1)$.

\begin{tabular}{cc}
\hline$F_{\text {in }} / F_{\text {wd }}$ & $F_{\text {in }} /\left(10^{12} \mathrm{erg} \mathrm{s}^{-1} \mathrm{~cm}^{-2}\right)$ \\
\hline 0.01 & 0.1 \\
0.08 & 0.8 \\
0.57 & 5.1 \\
1.47 & 13.3 \\
3.81 & 34.5 \\
9.87 & 89.6 \\
25.61 & 234.4 \\
60.39 & 547.9 \\
\hline
\end{tabular}

über den Bereich $F_{\text {in }} / F_{\text {wd }}=0.01-97$ und skalieren für die anderen Winkel mit $\mu$, wobei die obere Grenze kleinen Schwankungen unterliegt. Die Abbildungen 2.8 bis 2.12 dokumentieren die ausgehenden Flüsse $F_{\text {out }}$ einiger repräsentativer Modelle (Tabelle 2.4). 


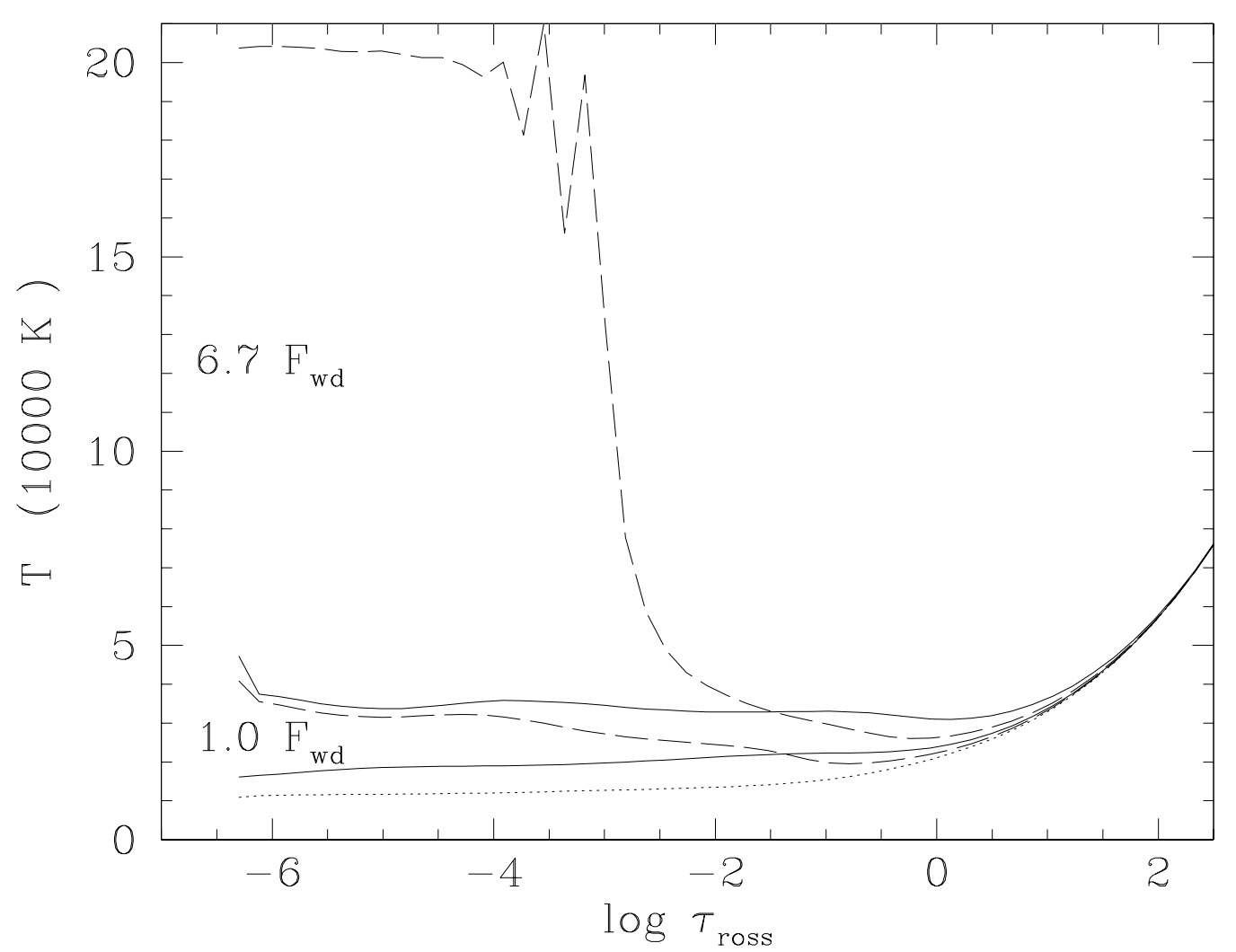

Abbildung 2.6: Temperaturschichtungen unter verschiedenen Bestrahlungflüssen und Bestrahlungswinkeln. Es sind zwei Kurvenpaare abgebildet, die jeweils zu einem Bestrahlungsfluss von $F_{\text {in }}=1 F_{\text {wd }}$ (oberes Kurvenpaar) und $F_{\text {in }}=6.7 F_{\text {wd }}$ gehören. Die gestrichelte Kurve entspricht flacher Bestrahlung $(\mu=0.1)$ und die durchgezogene senkrechter Einstrahlung $(\mu=1)$. 

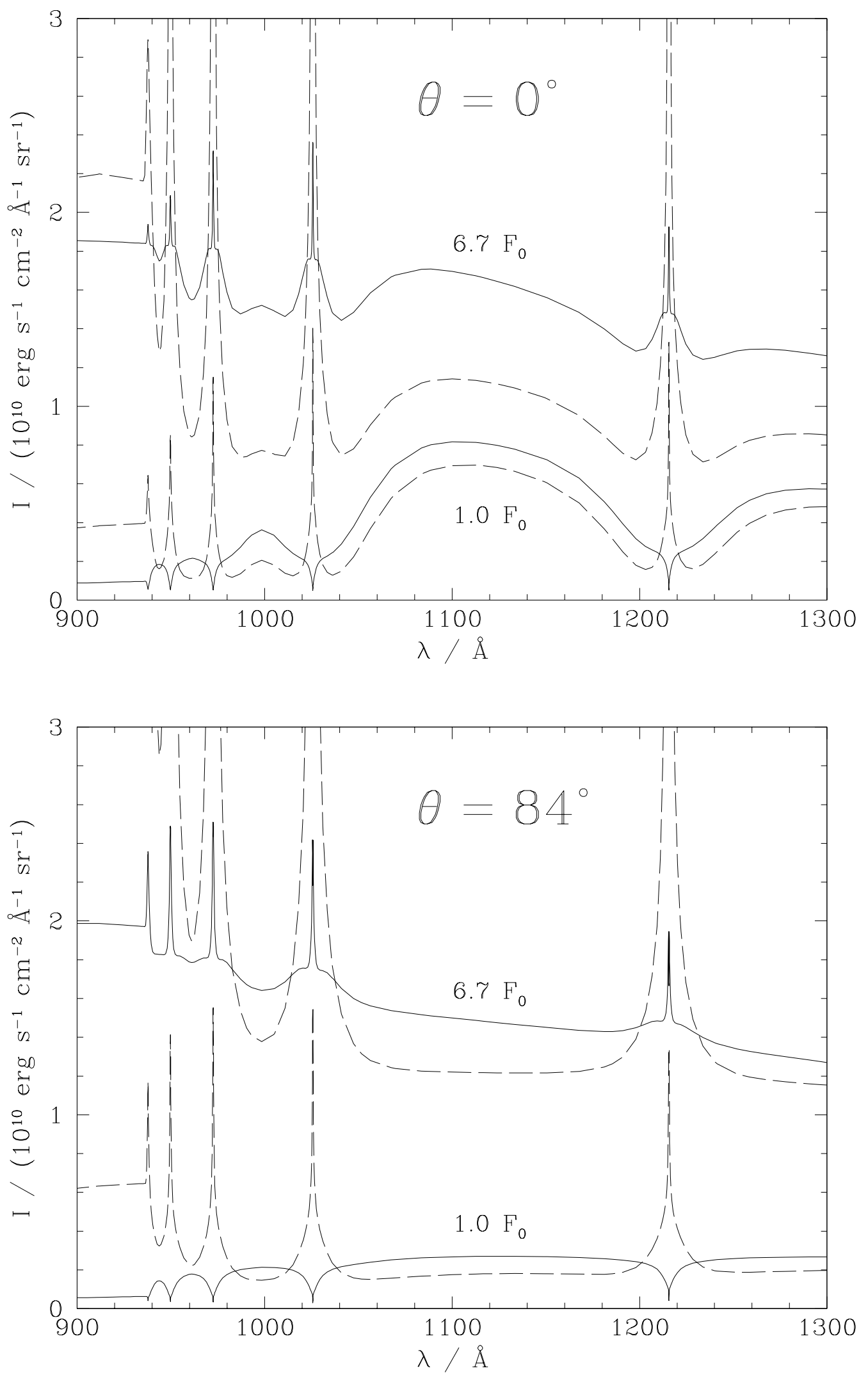

Abbildung 2.7: Vergleich winkelabhängiger Intensitäten $I$ unter verschiedenen Bestrahlungs- und Sichtwinkeln. Oben: senkrechter Sichtwinkel $\left(\theta=0^{\circ}\right)$, unten: flacher Sichtwinkel $\left(\theta=84^{\circ}\right)$. Die detaillierte Beschreibung befindet sich im Text. 

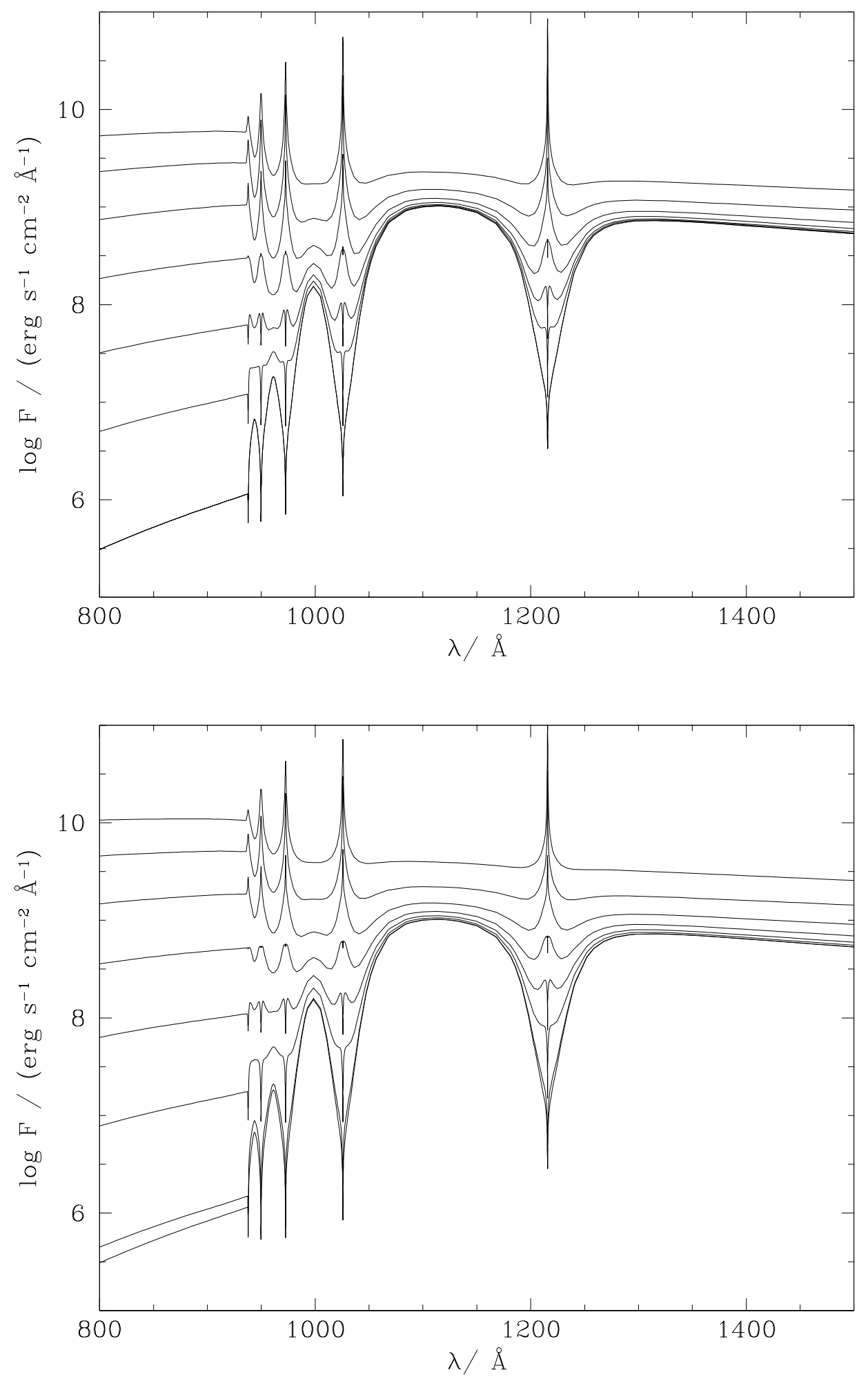

Abbildung 2.8: Ausgewählte Spektren aus dem Modellgitter für verschiedene Bestrahlungswinkel. Oben: $\mu=0.1$, unten: $\mu=0.2$. 

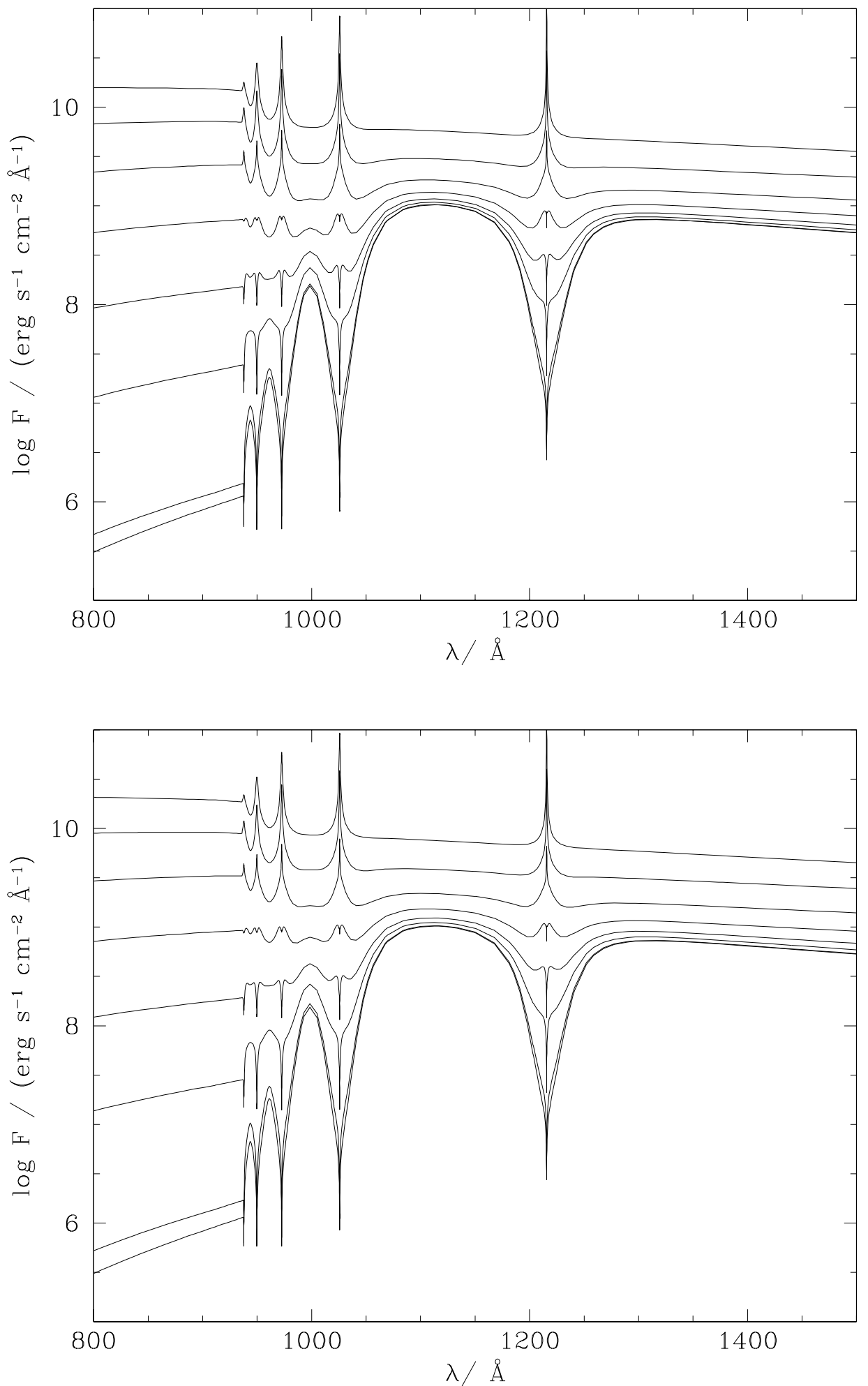

Abbildung 2.9: Oben: $\mu=0.3$, unten: $\mu=0.4$. 

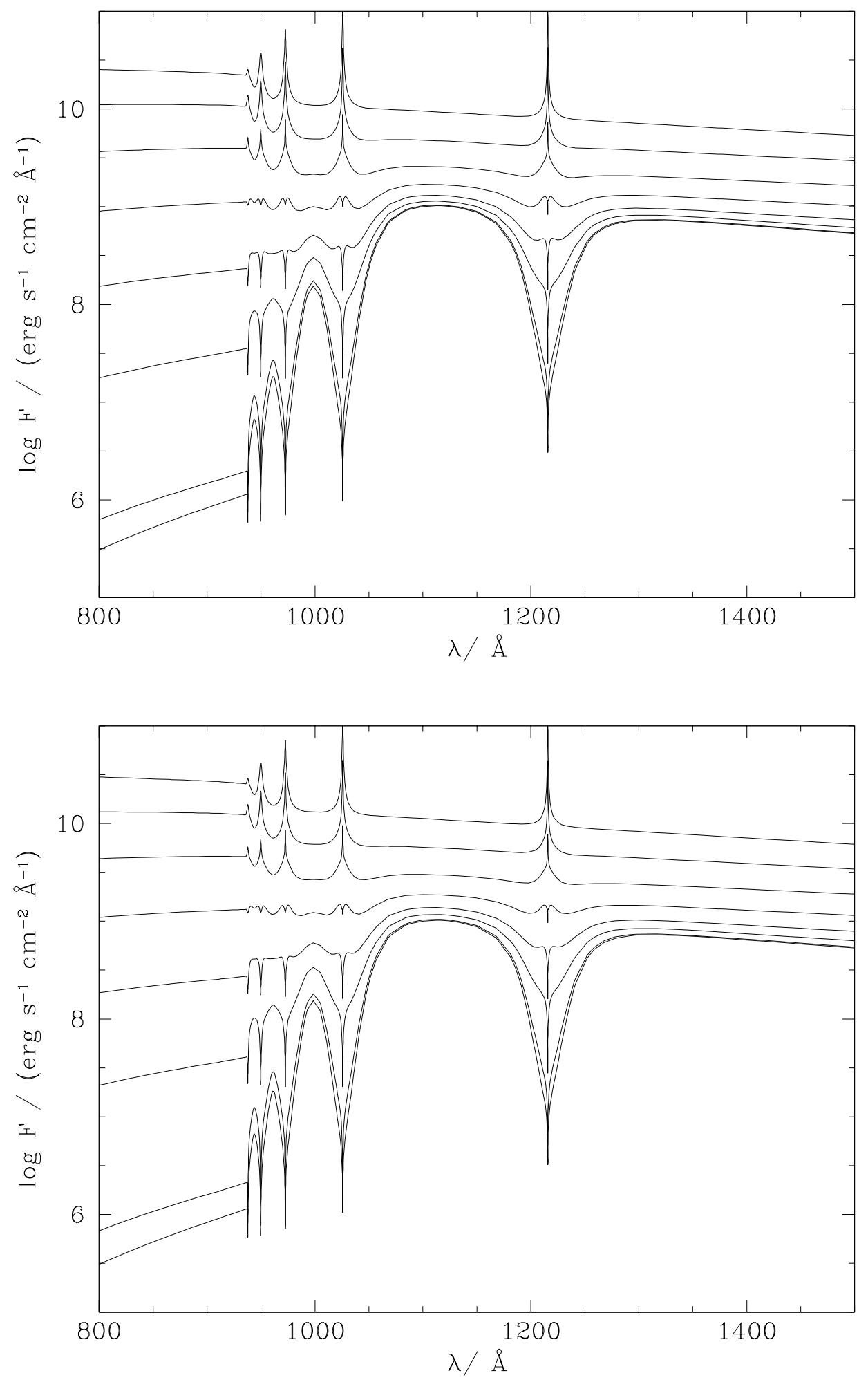

Abbildung 2.10: Oben: $\mu=0.5$, unten: $\mu=0.6$. 

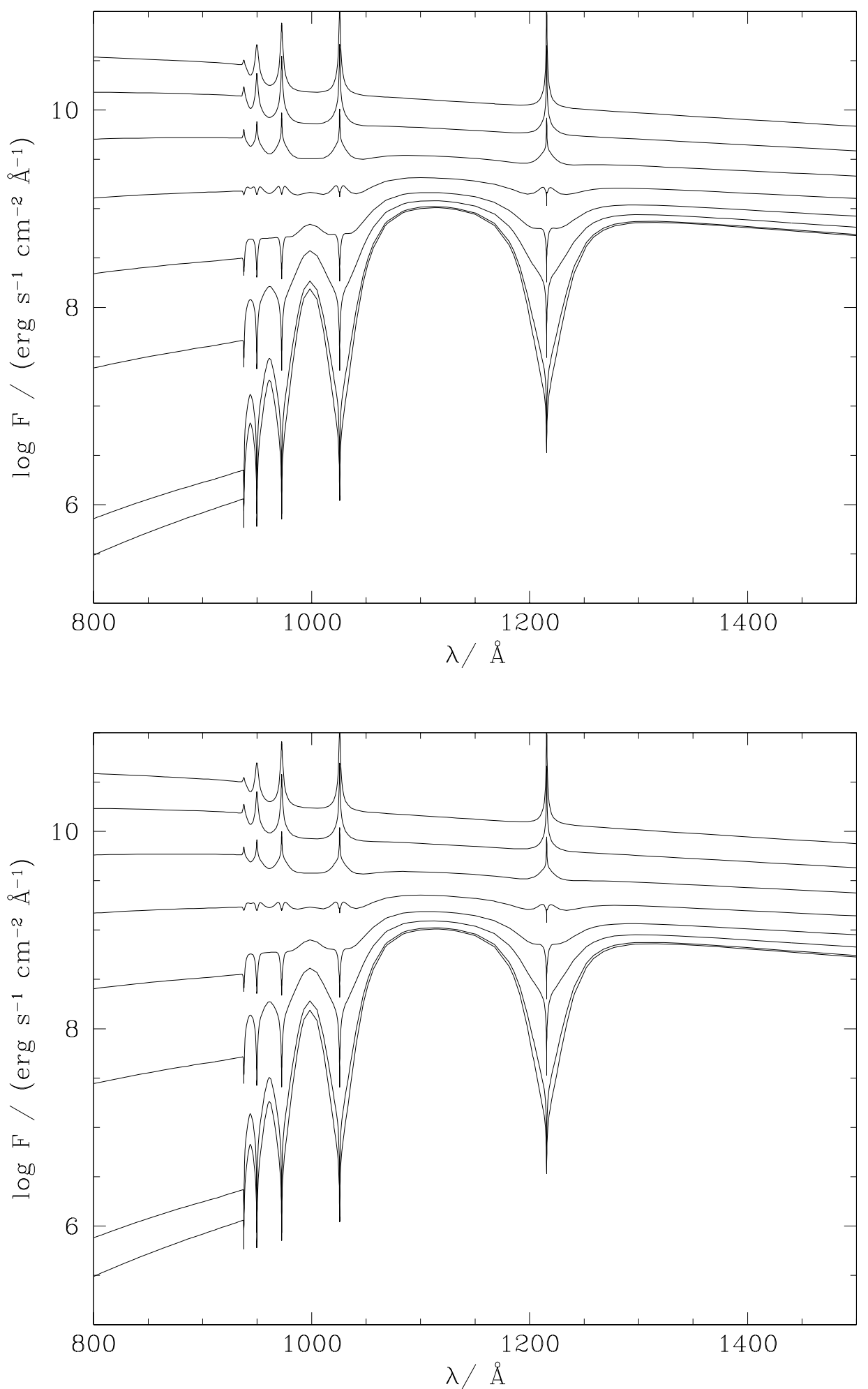

Abbildung 2.11: Oben: $\mu=0.7$, unten: $\mu=0.8$. 

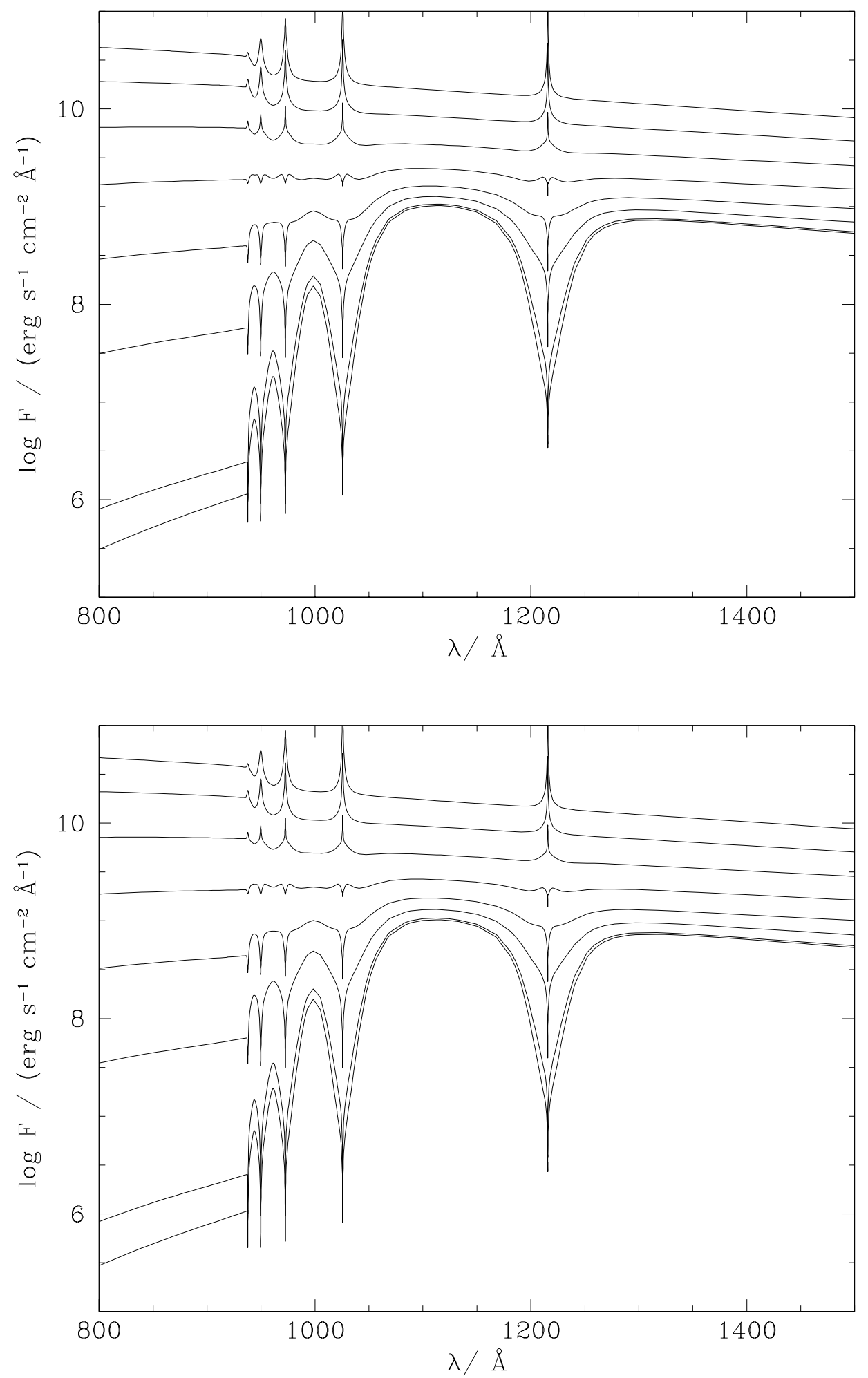

Abbildung 2.12: Oben: $\mu=0.9$, unten: $\mu=1.0$. 


\section{Kapitel 3}

\section{Dreidimensionale Modellierung eines bestrahlten Sterns auf Basis eindimensionaler winkelabhängiger Modellatmosphären}

\subsection{Methode}

\subsubsection{Ziele und Voraussetzungen}

Auf Basis der berechneten eindimensionalen Modellatmosphären soll nun ein dreidimensionales Modell eines bestrahlten Sterns entwickelt werden, um genaueren Aufschluss über die Struktur der geheizten Fläche der Atmosphäre und das resultierende Gesamtspektrum des Sterns zu erhalten.

Mit den eindimensionalen Berechnungen aus Abschnitt 2.2.2 existieren Modellatmosphären mit winkelabhängiger Einstrahlung von der Form

$$
I_{\mu_{i}}(\nu, \mu)=\delta\left(\mu-\mu_{i}\right) \cdot I_{\mu_{i}}(\nu)
$$

für ein diskretes Winkelgitter $\mu_{i}, i \in\left\{1, \ldots, N_{\mu}\right\}$. Das Modell soll so konstruiert sein, dass der Stern durch eine Menge von Flächenelementen dargestellt wird. Die Bestrahlungsquelle wird als Menge von Punktquellen modelliert, die über der Oberfläche des Sterns positioniert sind. Das Spektrum wird berechnet, indem zunächst für jedes Flächenelement der Bestrahlungsfluss und -winkel berechnet wird. Dadurch kann jedem Flächenelement eindeutig eine Modellatmosphäre zugeordnet werden, so dass durch Integration über alle Flächenelemente das Gesamtspektrum bestimmt werden kann. Weiterhin findet bedingt durch die Rotation des Gesamtsystems eine Variation des Spektrums statt, die durch die Angabe eines Phasenbereichs im Modell berücksichtigt werden soll.

Ein Problem ist, dass im Prinzip durch die Struktur der vorhandenen Modellatmosphären die Geometrie der Emissionsregion auf eine Punktquelle eingeschränkt 


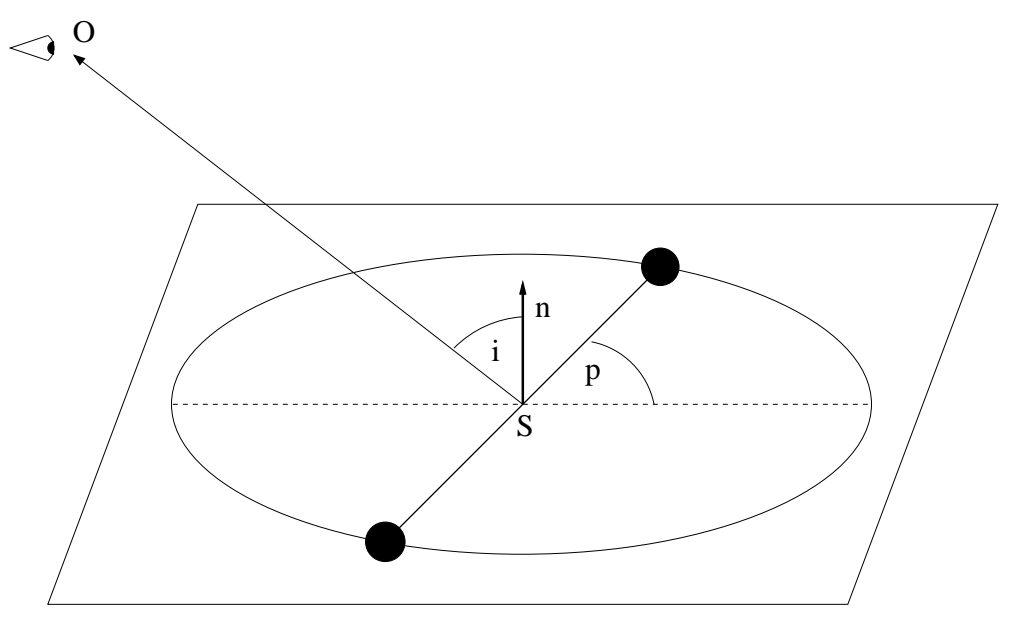

Abbildung 3.1: Veranschaulichung der Geometrieparameter eines CV-Systems.

ist. Wir werden jedoch versuchen, durch ein approximatives Verfahren die Geometrie der Emissionsregion zu erweitern, so dass auch ausgedehnte Emissionsregionen möglich sind. Da bei der Modellierung hauptsächlich jener Bereich der Sternoberfläche von Interesse ist, der für die Emissionsregion sichtbar ist, liegt es nahe, eine variable Gitterstruktur für die Kugeloberfläche zu verwenden, die im Bereich der Emissionregion eine feinere Auflösung besitzt.

\subsubsection{Systemgeometrie}

Die Spezifikation des Modells erfordert eine Anzahl von Parametern, die die Systemgeometrie eindeutig festlegen. Wir führen hier nur die physikalisch wichtigsten an. Eine vollständige Referenz findet sich im Anhang A.

- inclination

Da es sich bei einem CV-System um ein Doppelsternsystem handelt, findet die Rotation der beiden Komponenten in einer Ebene $E$ um den gemeinsamen Schwerpunkt S statt. Der Beobachter befinde sich am Punkt O. Der Normalenvektor $\vec{n}$ bildet mit der Verbindungslinie $O S$ den Winkel $i$. Den Winkel $i$ bezeichnet man als Inklination des Systems. Abbildung 3.1 veranschaulicht die Geometrie des Systems.

- distance

Der Abstand des Systems zum Beobachter.

- wd_radius

Für das Modell ist im Wesentlichen nur der Primärstern (der Weiße Zwerg) des Sytems interessant, dessen Radius $r_{\text {wd }}$ durch diesen Parameter spezifiziert wird. 
- phase_start, phase_end, phase_step

Die Phase des Modells entspricht der Orbitalphase des CV-Systems. phase_start und phase_end benennen die Start- und Endphase des Modells, wobei phase_step die Schrittweite definiert.

\subsubsection{Weltobjekte}

Das Modell besteht geometrisch aus einer Anzahl von Objekten, die in einem dreidimensionalen Raum positioniert sind. Es werden nun die einzelnen Typen beschrieben, die relevant sind. Da der Code in der Sprache C implementiert ist, entsprechen die Namen direkt den C-typedef-Definitionen im Code.

\section{Der Primärstern: sphere_t}

Der Typ sphere_t repräsentiert den Primärstern (den Weißen Zwerg). Die Fläche einer Kugel mit dem Radius $r_{\mathrm{wd}} \in \mathbb{R}^{+}$kann parametrisiert werden durch Einführung von Koordinaten $(\theta, \varphi)$, mit $\theta \in] 0, \pi]$ und $\varphi \in] 0,2 \pi]$ :

$$
\vec{x}(\theta, \varphi)=r_{\mathrm{wd}}\left(\begin{array}{l}
\sin \theta \cos \varphi \\
\sin \theta \sin \varphi \\
\cos \theta
\end{array}\right)
$$

Prinzipiell wäre es geschickt, den Pol $(0,0)$ mit dem Zentrum der Emissionsregion zu identifizieren, wenn man eine eine radialsymmetrische Verteilung voraussetzt. Da jedoch die Geometrie möglichst allgemein gehalten werden soll, und eine symmetrische Verteilung nicht unbedingt vorausgesetzt werden soll, wird die Polachse mit der Rotationsachse identifiziert.

Die Diskretisierung des Parameterraums erfolgt durch Vorgabe der Anzahl der Diskretisierungspunkte $N_{\varphi}$ und $N_{\theta}$ und ist definiert durch

$$
\begin{aligned}
\theta_{i} & =\frac{\pi}{N_{\theta}} i, \quad i \in\left\{0,1, \ldots, N_{\theta}-1\right\} \\
\varphi_{i} & =\frac{2 \pi}{N_{\varphi}} i, \quad i \in\left\{0,1, \ldots, N_{\varphi}-1\right\} .
\end{aligned}
$$

Weiterhin gilt die Identifikation

$$
\theta_{N_{\theta}}=\theta_{0}, \quad \varphi_{N_{\varphi}}=\varphi_{0}
$$

Das Gittermodell der Kugel erfolgt nun durch Konstruktion von Flächenelementen (face_t-Objekten) in zwei Schritten:

1. Konstruktion der Flächenelemente aus dem primären Gitter. Für Flächenelemente, die einen Polpunkt enthalten, werden Dreiecke aus den Punkten $P_{0}, P_{1}$ und $P_{2}$ konstruiert. 
Oberer Pol $(0,0)$ :

$$
\left(\begin{array}{l}
P_{0} \\
P_{1} \\
P_{2}
\end{array}\right)=\left(\begin{array}{l}
(0,0) \\
\left(\theta_{1}, \varphi_{i}\right) \\
\left(\theta_{1}, \varphi_{i+1}\right)
\end{array}\right) \quad, \forall i \in\left\{0,1,2, \ldots, N_{\varphi}-1\right\}
$$

Unterer Pol $(\pi, 0)$ :

$$
\left(\begin{array}{l}
P_{0} \\
P_{1} \\
P_{2}
\end{array}\right)=\left(\begin{array}{l}
\left(\theta_{N_{\theta}-1}, \varphi_{i}\right) \\
(\pi, 0) \\
\left(\theta_{N_{\theta}-1}, \varphi_{i+1}\right)
\end{array}\right) \quad, \forall i \in\left\{0,1,2, \ldots, N_{\varphi}-1\right\}
$$

Für den mittleren Teil werden trapezförmige Flächenelemente aus den 4 Punkten $P_{0}, P_{1}, P_{2}$ und $P_{3}$ konstruiert.

$$
\left(\begin{array}{l}
P_{0} \\
P_{1} \\
P_{2} \\
P_{3}
\end{array}\right)=\left(\begin{array}{l}
\left(\theta_{j}, \varphi_{i}\right) \\
\left(\theta_{j+1}, \varphi_{i}\right) \\
\left(\theta_{j+1}, \varphi_{i+1}\right) \\
\left(\theta_{j}, \varphi_{i}\right)
\end{array}\right) \quad, \quad \forall i \in\left\{0,1,2, \ldots, N_{\varphi}-1\right\}
$$

2. Nach Positionierung der Emissionsregion erfolgt eine Verfeinerung des Gitters. Jedes Flächenelement, das von mindestens einer Punktquelle bestrahlt wird, wird in mehrere kleinere Flächenelemente aufgeteilt.

Bei der Verfeinerung wird ausgenutzt, dass alle Gitterpunkte auf einer Kugeloberfläche liegen, deren Mittelpunkt im Ursprung liegt. Die Strecke zwischen zwei Punkten $A$ und $B$, die zu den Definitionspunkten eines Flächenelementes gehören, wird halbiert. Die Strecke zwischen dem neu entstandenen Punkt $P$ und dem Ursprung wird skaliert, so dass ein neuer Punkt $C$ auf der Kugeloberfläche entsteht (Abbildung 3.2 ).

Ein Flächenelement wird so durch vier neue kleinere Flächenelemente ersetzt. Abbildungen 3.3 und 3.4 veranschaulichen diesen Prozess.

\section{Punktquelle: sphere_radiation_source_t}

Dieser Objekttyp beschreibt eine punktförmige isotrope Strahlungsquelle. Ihre Eigenschaften sind definiert durch die Position $(x, y, z)$ im Raum und physikalisch durch die Leuchtkraft $L$. Punktquellen werden nicht direkt erzeugt, sondern vermittelt durch ein Objekt des Typs sphere_emission_region_t, welches die Emissionsregion repräsentiert.

\section{Die Emissionsregion: sphere_emission_region_t}

Eine sphere_radiation_source_t beschreibt lediglich eine punktförmige Quelle. Es wäre jedoch wünschenswert, zumindest approximativ ausgedehnte Strahlungsquellen - eine Emissionsregion - im Modell behandeln zu können. 


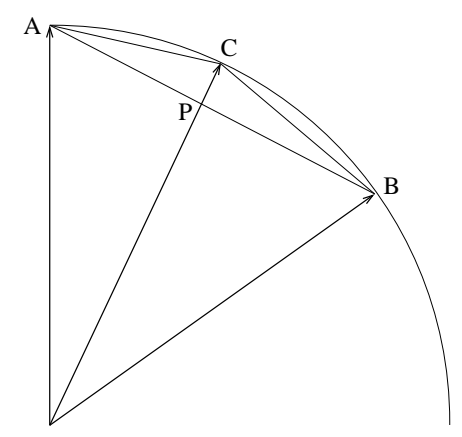

Abbildung 3.2: Auswahl der neuen Punkte des Verfeinerungsalgorithmus für die Flächenelemente. Bei der Teilung der Flächenelemente werden die neuen Punkte der geteilten Seiten an die Kugeloberfläche angepasst.
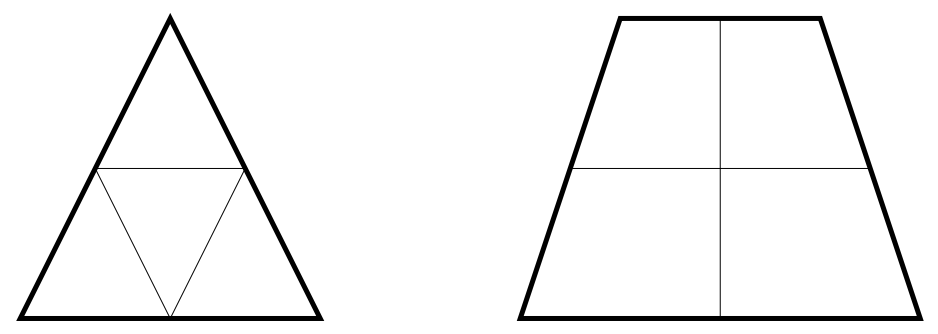

Abbildung 3.3: Bei der Verfeinerung wird ein Flächenelement in vier kleinere aufgeteilt und durch diese ersetzt. 

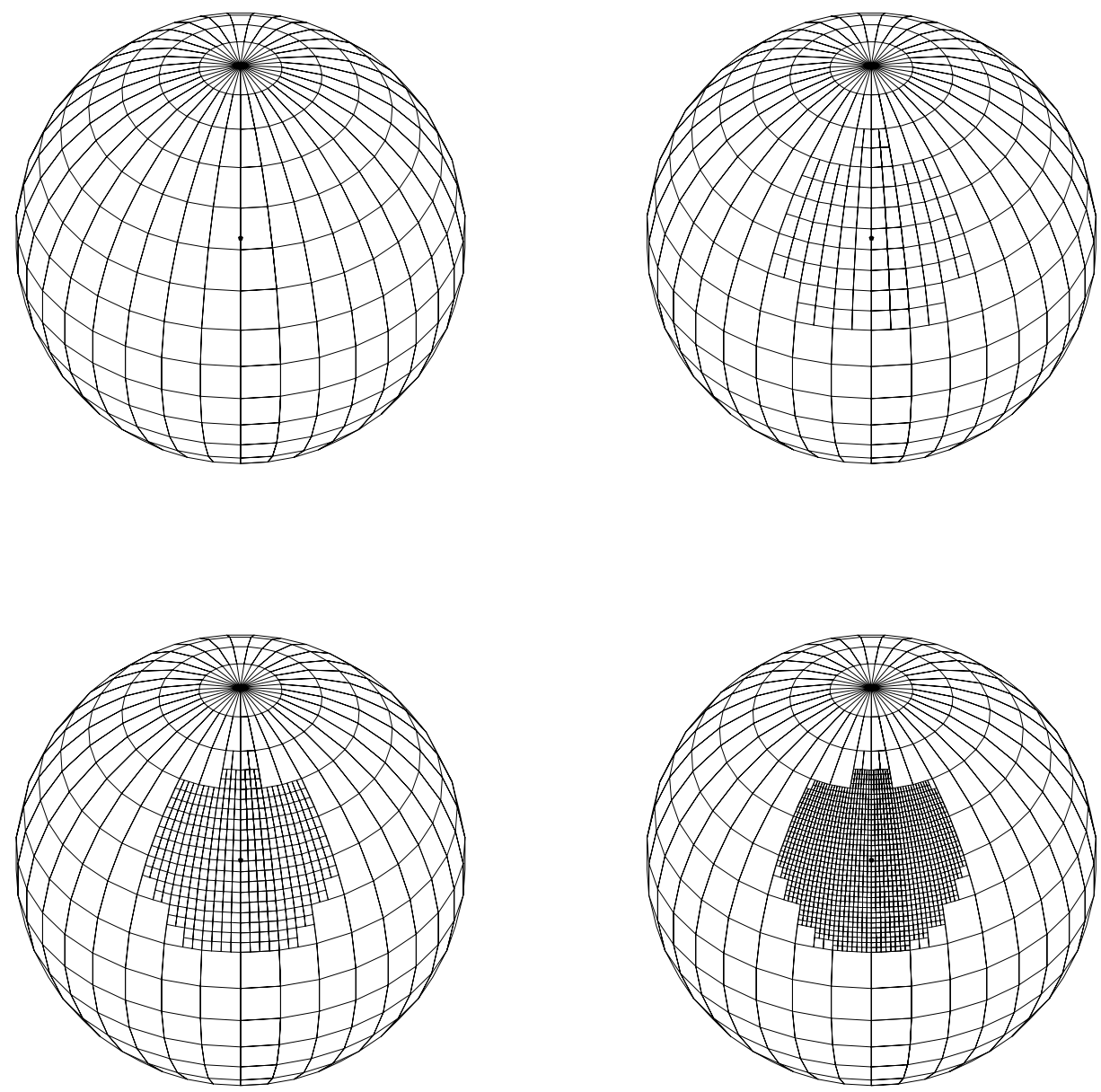

Abbildung 3.4: Man sieht hier die iterative Verfeinerung des Gitters für Flächenelemente, die für die Punktquelle sichtbar sind. Links oben ist das primäre Gitter abgebildet, der Punkt repräsentiert die Strahlungsquelle, die in einer Höhe von 0.1 Kugelradien über der Oberfläche schwebt. Die Bilder oben rechts und unten zeigen die Verfeinerung bei ein-, zwei- und dreimaliger Iteration.

Bei der Emissionsregion handelt es sich um einen Container 1 , der eine Menge von Punktquellen mit einer bestimmten Anordnungsgeometrie kapselt und eine Schnittstelle zu diesen liefert. Eine Emissionsregion wird definiert durch ihren Typ, der die Anordnungsgeometrie beschreibt, ihre Position und ihre Leuchtkraft. Die Position der Emissionsregion wird spezifiziert durch die Angabe folgender Parameter:

- source_theta

${ }^{1}$ Ein Container ist ein Objekt, welches im Wesentlichen die Aufgabe besitzt, andere Objekte zu enthalten. 
Dieser Parameter bestimmt die Komplementbreite („colatitude“) der Emissionsregion. Darunter versteht man die Winkelkoordinate $\theta_{\text {er }}$ des Zentrums der Emissionsregion ausgehend von der Rotationsachse des Weißen Zwergs.

- source_phi

Die Winkelkoordinate $\varphi_{\text {er }}$ beschreibt den Winkelversatz des Zentrums der Emissionsregion in Rotationsrichtung.

- source_height

Die Höhe der Emissionsregion über der Oberfläche in Einheiten von $r_{\text {wd }}$ wird durch diesen Parameter definiert.

Die Anordnungsgeometrie gibt die relative Lage der Punktquellen zueinander an. Dabei sind je nach Typ weitere geometrische Parameter zur genauen Spezifikation notwendig. Der Typ wird durch den Parameter source_type festgelegt.

- Typ: point

Beschreibt eine einfache isotrop strahlende Punktquelle. Hier sind keine weiteren Parameter notwendig.

- Typ: circular_area

Hier sind die einzelnen Punktquellen auf einer Kreisfläche positioniert. Diese Emissionsregion wird modelliert aus einzelnen Kreisen, auf denen jeweils die Punktquellen verteilt werden. Dabei nimmt die Anzahl der Quellen von innen nach außen linear zu. Zur genauen Spezifikation der Emissionsregion sind noch folgende Parameter notwendig:

- source_circle_radius

Der Radius $r_{\text {er }}$ der Emissionsregion, anzugeben in Einheiten des Sternradius, wird durch diesen Parameter angegeben.

- source_ncircles

Da die Kreisfläche aus einer Anzahl von konzentrischen Kreisen modelliert wird, ist die Anzahl der Kreise durch diesen Parameter anzugeben.

- source_npoints

Gesamtzahl der Punktquellen der Emissionsregion.

Die Gesamtleuchtkraft $L$ für die Emissionsregion wird durch den Parameter source_luminosity / $\mathrm{ergs}^{-1}$ festgelegt. Die Leuchtkraft $L$ wird auf die $N$ Punktquellen verteilt, so dass gilt:

$$
L=\sum_{i=1}^{N} L_{i}
$$


Die Verteilung wird durch den Parameter source_luminosity_distribution festgelegt. Hier ist allerdings derzeit nur eine homogene Verteilung (homogeneous) möglich, so dass die Leuchtkraft einer Punktquelle gegeben ist durch

$$
L_{i}=\frac{L}{N}
$$

\subsubsection{Berechnung der physikalischen Größen}

Im folgenden sei vorausgesetzt, dass der Modellstern $\mathcal{S}$ mit dem Radius $r_{\text {wd }}$ durch eine Menge von $N_{\mathcal{S}}$ Flächenelementen dargestellt ist. Ein Flächenelement $\mathcal{A} \equiv(\vec{A}, \vec{q})$ besteht dabei aus einem Darstellungsvektor $\vec{A}$ und einem Positionsvektor $\vec{q}$. Dabei entspricht die Richtung von $\vec{A}$ der Normalen und der Betrag $|\vec{A}|$ dem Flächeninhalt des Flächenelementes. Der Vektor $\vec{q}$ entspricht der Position.

$$
\mathcal{S}=\left\{\left(\vec{A}_{i}, \vec{q}_{i}\right) \in \mathbb{R}^{3} \times \mathbb{R}^{3} \mid i \in\left\{1, \ldots, N_{\mathcal{S}}\right\}\right\}
$$

Die Emissionsregion $\mathcal{E}$ besteht aus einer Menge von $N_{\mathcal{E}}$ Punktquellen. Dabei ist eine Punktquelle $\mathcal{P} \equiv(\vec{p}, L)$ gegeben durch die Position $\vec{p}_{j}$ und die Leuchtkraft $L_{j}$.

$$
\mathcal{E}=\left\{\left(\vec{p}_{i}, L_{i}\right) \in \mathbb{R}^{3} \times \mathbb{R}_{0}^{+} \mid i \in\left\{1, \ldots, N_{\mathcal{E}}\right\}\right\}
$$

Weiterhin bezeichnen wir mit $h$ die Höhe einer Punktquelle über der Oberfläche des Modellsterns

$$
h \equiv h\left(\vec{p}_{i}\right):=\left|\vec{p}_{i}\right|-r_{\mathrm{wd}}
$$

und mit $\alpha$ den Winkel zwischen dem Positionsvektor $\vec{p}_{j}$ einer Punktquelle und dem Flächenelementvektor $\vec{A}_{i}$

$$
\alpha \equiv \alpha\left(\vec{A}_{i}, \vec{p}_{j}\right):=\angle\left(\vec{A}_{i}, \vec{p}_{j}\right)
$$

\section{Fluss}

Nun muss der Bestrahlungsfluss der Emissionsregion $\mathcal{E}$ bestimmt werden, der ein Flächenelement erreicht. Der einfallende Strahlungsfluss ${ }^{2} F_{\text {in }}(\mathcal{E}, \mathcal{A})$, der ein Flächenelement $\mathcal{A}$ durchdringt, berechnet sich aus der Summe aller Flüsse $F_{\text {in }}\left(\mathcal{P}_{j}, \mathcal{A}\right)$, die den einzelnen Punktquellen $\mathcal{P}_{j}$ der Emissionsregion $\mathcal{E}$, die für das jeweilige Flächenelement sichtbar sind, entspringen.

\footnotetext{
${ }^{2}$ Insofern die Flussgrößen keine angegebene Frequenzabhängigkeit besitzen, handelt es sich um den frequenzintegrierten Fluss: $F=\int_{0}^{\infty} F(\nu) d \nu$
} 


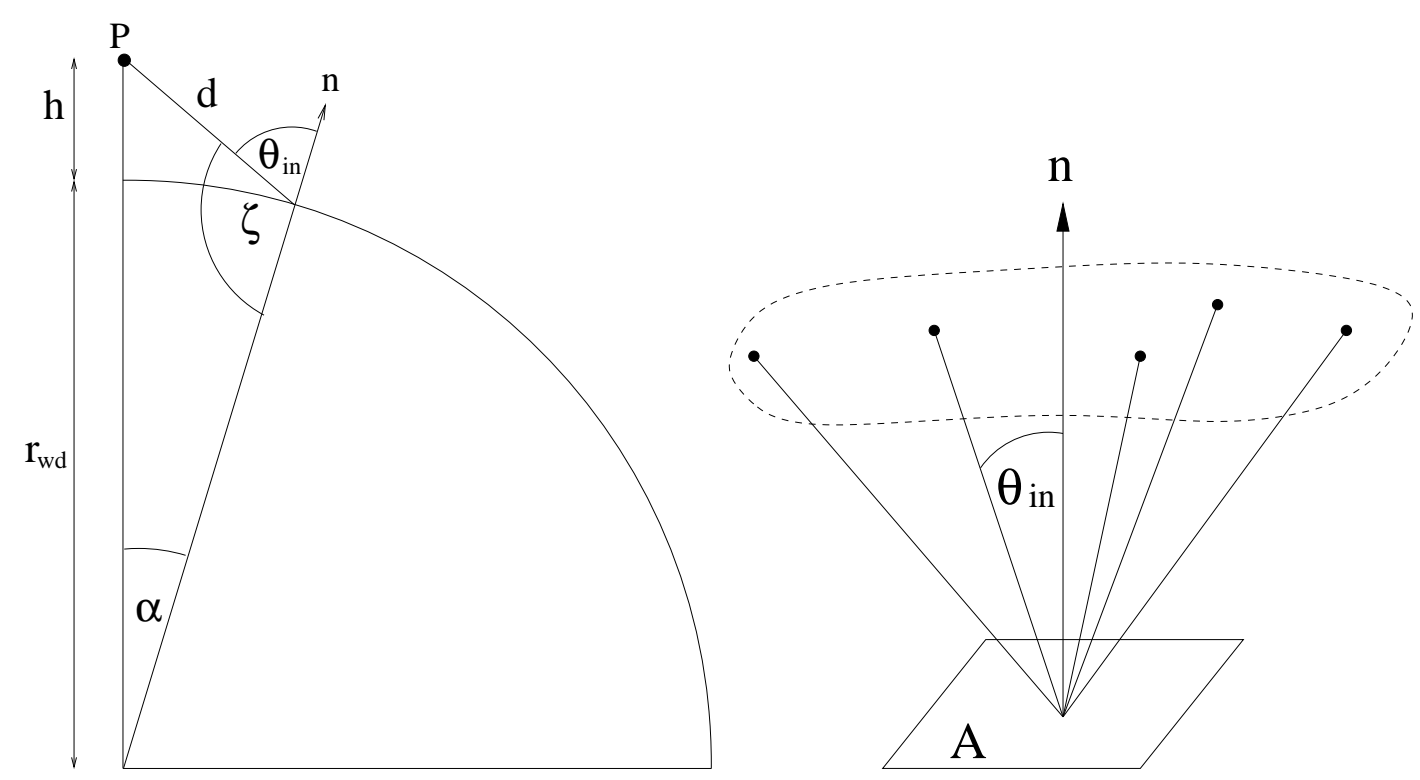

Abbildung 3.5: Links: Geometrische Größen zur Berechnung des Flusses für ein Flächenelement. Eine Punktquelle $P$ schwebt in der Höhe $h$ über der Oberfläche des Weißen Zwerges mit dem Radius $r_{\text {wd }}$. Ein Flächenelement $A$ mit dem Normalenvektor $\vec{n}$ bildet mit dem Positionsvektor der Punktquelle den Winkel $\alpha$. Die Entfernung der Punktquelle $P$ zum Flächenelement $A$ wird mit $d$ bezeichnet. $\theta_{\text {in }}$ ist der Bestrahlungswinkel. Rechts: Darstellung einer ausgedehnten Emissionsregion, die aus mehreren Punktquellen besteht.

Sichtbare Flächenelemente Für eine Punktquelle $\mathcal{P}$ gibt es einen maximalen Winkel $\alpha_{\max }$, so dass für alle Flächenelemente $\mathcal{A}$ mit $\alpha<\alpha_{\max } \mathcal{P}$ sichtbar ist. Dieser Winkel bestimmt sich aus der Bedingung, dass der Bestrahlungswinkel $\theta_{\text {in }}=90^{\circ}$ ist (siehe auch Abb. 3.5):

$$
\alpha_{\max } \equiv \alpha_{\max }(h)=\arccos \left(\frac{r_{\mathrm{wd}}}{r_{\mathrm{wd}}+h}\right)
$$

Der Gesamtbestrahlungsfluss lässt sich dann folgendermaßen ausdrücken:

$$
F_{\text {in }}(\mathcal{E}, \mathcal{A})=\sum_{k=1}^{N_{\mathcal{E}}} \Theta\left(\alpha_{\max }-\alpha\right) F_{\text {in }}\left(\mathcal{P}_{k}, \mathcal{A}\right)
$$

wobei $\Theta$ definiert ist durch

$$
\Theta(x):=\left\{\begin{array}{ll}
1 & \text { für } x>0 \\
0 & \text { für } x<0
\end{array} .\right.
$$


Bestrahlungswinkel für ein Flächenelement und eine Punktquelle Der Bestrahlungswinkel $\theta_{\text {in }}(\mathcal{P}, \mathcal{A})$ ist definiert durch den Winkel zwischen dem Normalenvektor $\vec{n}=\vec{A} /|\vec{A}|$ eines Flächenelements $\mathcal{A}$ und dem Vektor $(\vec{p}-\vec{q})$ (der Verbindungslinie von Punktquelle und Flächenelementposition). Er kann dabei bei gegebener Punktquelle direkt als Funktion $\theta_{\text {in }} \equiv \theta_{\text {in }}(\alpha)$ des Winkels $\alpha$ berechnet werden. Die Berechnung der Strecke $d$ ergibt (siehe Abbildung 3.5)

$$
\begin{aligned}
d^{2} & =r_{\mathrm{wd}}^{2}+\left(r_{\mathrm{wd}}+h\right)^{2}-2 r_{\mathrm{wd}}\left(r_{\mathrm{wd}}+h\right) \cos \alpha \\
& =2 r_{\mathrm{wd}}\left(r_{\mathrm{wd}}+h\right)(1-\cos \alpha) \\
\Rightarrow d(h, \alpha) & =\sqrt{2 r_{\mathrm{wd}}\left(r_{\mathrm{wd}}+h\right)(1-\cos \alpha)} .
\end{aligned}
$$

Für $\zeta$ bekommen wir

$$
\begin{aligned}
& \left(h+r_{\mathrm{wd}}\right)^{2}=d^{2}+r_{\mathrm{wd}}^{2}-2 d r_{\mathrm{wd}} \cos \zeta \\
& \Rightarrow \zeta(h, \alpha)=\zeta(h, d(\alpha))=\arccos \left[\frac{1}{2}\left(\frac{d}{r_{\mathrm{wd}}}-\frac{h^{2}}{d r_{\mathrm{wd}}}-\frac{2 h}{d}\right)\right] .
\end{aligned}
$$

Der Bestrahlungswinkel $\theta_{\text {in }}$ ist dann

$$
\theta_{\text {in }}(\mathcal{P}, \mathcal{A})=\pi-\zeta(h, \alpha)
$$

Bestrahlungswinkel für ein Flächenelement und eine Emissionsregion Es ist klar, dass aufgrund der vorliegenden Modellatmosphärendatenbank nur Modelle exakt behandelt werden können, die von einer einzigen Punktquelle bestrahlt werden. Das liegt daran, dass für ein bestimmtes Flächenelement nur eine Bestrahlung unter einem Winkel existiert. Bei einem Modell einer Emissionsregion gäbe es jedoch mehrere Bestrahlungswinkel von jeweils verschiedenen Punktquellen. Um ausgedehnte Emissionsregionen zumindest approximativ behandeln zu können, wird ein gemittelter flussgewichteter Bestrahlungswinkel

$$
\left\langle\theta_{\text {in }}\right\rangle(\mathcal{E}, \mathcal{A}):=\sum_{i=1}^{N_{\mathcal{E}}} \frac{\theta_{\text {in }}\left(\mathcal{P}_{i}, \mathcal{A}\right) \cdot F_{\text {in }}\left(\nu, \mathcal{P}_{i}, \mathcal{A}\right)}{F_{\text {in }}(\nu, \mathcal{E}, \mathcal{A})}
$$

definiert.

Fluss $F_{\text {in }}(\mathcal{P}, \mathcal{A})$ für ein Flächenelement Die Punktquelle $\mathcal{P}$ strahlt isotrop mit der Leuchtkraft $L$. In einer Entfernung $d$, die durch Gleichung (3.19) gegeben ist, entspricht dies dem Fluss

$$
F_{\text {radial }}(L, d)=\frac{L}{4 \pi d^{2}} .
$$

Die Richtung des Flusses entspricht dabei der radialen Richtung bezüglich der Punktquelle. Dabei handelt es sich um den Fluss, der durch ein Flächenelement 
tangential zur Kugelfläche mit dem Radius $d$ um $\vec{p}$ fließen würde. Der Fluss durch das Flächenelement $\mathcal{A}$ ergibt sich durch die Projektion auf den Normalenvektor des Flächenelements:

$$
F_{\text {in }}(\mathcal{P}, \mathcal{A})=F_{\text {radial }}(L, d) \cos \theta_{\text {in }}=\frac{L}{4 \pi d^{2}} \cos \theta_{\text {in }}
$$

\subsubsection{Berechnung des Gesamtspektrums}

Mit diesen Voraussetzungen kann nun das Spektrum des Modellsterns berechnet werden. Während bei einem unbestrahlten Stern der Strahlungsfluss in der äußersten Schicht $F(\nu)$ identifiziert werden kann mit dem beobachteten Spektrum, gilt dies nicht mehr für einen bestrahlten Stern, da dort $F \equiv \int_{0}^{\infty} F(\nu) d \nu=F_{\text {in }}+F_{\text {out }}$ gilt. Die Bestrahlung $F_{\text {in }}$ ist jedoch für den Beobachter nicht sichtbar. Aus diesem Grund ist lediglich der ausgehende Fluss $F_{\text {out }}$ mit dem Spektrum zu identifizieren.

Auswahl der Modellatmosphäre Für ein Flächenelement $\mathcal{A}$ sind der Gesamtbestrahlungsfluss $F_{\text {in }}(\mathcal{E}, \mathcal{A})$ durch $(3.17)$ und der Bestrahlungswinkel $\theta_{\text {in }} \equiv\left\langle\theta_{\text {in }}\right\rangle(\mathcal{E}, \mathcal{A})$ durch $(3.22)$ gegeben. Mit $\mu_{\text {in }}$ bezeichnen wir im Folgenden $\mu_{\text {in }}=\cos \left(\theta_{\text {in }}\right)$.

Da die Menge der vorhandenen Winkel $\left\{\mu_{i} \mid i \in 1, \ldots N_{\mu}\right\}$ diskret ist, wird derjenige Winkel mit dem geringsten Abstand zu $\mu_{\text {in }}$ ausgewählt.

$$
\mu_{\mathrm{in}, i}=\min \left\{\left|\mu_{\mathrm{in}}-\mu_{i}\right| \quad \mid i \in 1, \ldots, N_{\mu}\right\} .
$$

Durch den Index $i$ ist die Auswahl des Winkels vollständig bestimmt.

Ebenso ist eine diskrete Menge von Bestrahlungsflüssen $\left\{F_{i}\right\}$ vorhanden. Ana$\log$ zur Selektion des Winkels wird hier der Fluss aus dem Gitter ausgewählt, der den geringsten Abstand zu $F_{\text {in }}(\mathcal{E}, \mathcal{A})$ besitzt.

$$
F_{\text {in }}=\min \left\{\left|F_{i}-F_{\text {in }}(\mathcal{E}, \mathcal{A})\right|\right\} .
$$

Diese 2 Größen bestimmen eindeutig die zu wählende Modellatmosphäre. Dadurch ist der ausgehende Fluss $F_{\text {out }}(\nu, \mathcal{E}, \mathcal{A})$ bestimmt.

Beobachter und sichtbare Flächenelemente Der Beobachter befinde sich im Abstand $d$, mit $d \gg r_{\text {wd }}$. Er kann spezifiziert werden durch eine Beobachterrichtung $\vec{n}_{\text {obs. }}$. Die Ebene senkrecht zu $\vec{n}_{\text {obs }}$ ist die Projektionsfläche $E_{\text {obs }}$. Mit $\theta_{\text {out }}$ bezeichnen wir den Winkel zwischen Beobachterrichtung $\vec{n}_{\text {obs }}$ und Flächenelementvektor $\vec{A}$ (Abb. 3.6):

$$
\theta_{\text {out }} \equiv \theta_{\text {out }}\left(\vec{n}_{\text {obs }}, \vec{A}\right):=\angle\left(\vec{n}_{\text {obs }}, \vec{A}\right)
$$

Wir definieren die Menge $\mathcal{V}$ aller für den Beobachter sichtbaren Flächenelemente durch

$$
\mathcal{V}:=\left\{\mathcal{A} \equiv(\vec{A}, \vec{q}) \in \mathcal{S} \quad \mid \quad \theta_{\text {out }}<\frac{\pi}{2}\right\} .
$$




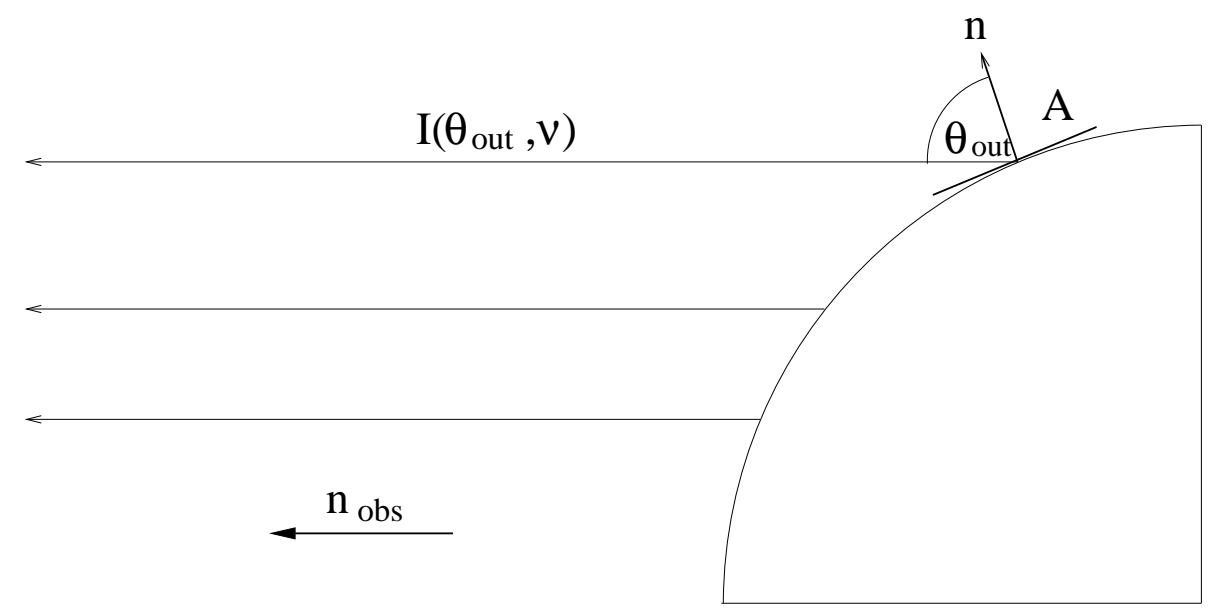

Abbildung 3.6: Definition des Winkels $\theta_{\text {out }}$ und Bestimmung der ausgehenden Intensität $I\left(\theta_{\text {out }}, \nu\right)$.

Effektive Fläche und Fluss Die effektive Fläche $A_{\text {eff }}$ eines Flächenelementes $\mathcal{A}$ ist gegeben durch die Projektion auf die Ebene $E_{\text {obs }}$ :

$$
A_{\text {eff }}:=\vec{A} \cdot \vec{n}_{\text {obs }}=|\vec{A}| \cos \theta_{\text {out }} .
$$

Die Summe aller effektiven Flächen wird mit $A_{\text {total }}$ bezeichnet:

$$
A_{\text {total }}=\sum_{\mathcal{A} \in \mathcal{V}} A_{\text {eff }} \text {. }
$$

Entsprechend ist der effektive Fluss $F_{\text {eff }}(\nu, \mathcal{E}, \mathcal{A})$ die Projektion des Flusses $F_{\text {out }}(\nu, \mathcal{E}, \mathcal{A})$, der bez. der Richtung von $\vec{A}$ des Flächenelementes $\mathcal{A}$ gilt, auf die Beobachterrichtung $\vec{n}_{\text {obs }}$

$$
F_{\text {eff }}(\nu, \mathcal{E}, \mathcal{A})=F_{\text {out }}(\nu, \mathcal{E}, \mathcal{A}) \cos \theta_{\text {out }}
$$

Spektrum Die exakte Berechnung des Spektrums erfolgt unter Benutzung der gerichteten Intensitäten $I(\theta, \nu)$, wobei auch Randeffekte berücksichtigt werden können. Für den von einem Flächenelement $\mathcal{A}$ beim Beobachter in der Entfernung $d$ ankommenden Fluss $F_{\text {obs }}(\nu)$ gilt unter der Voraussetzung, dass die Intensität über das Flächenelement örtlich konstant ist, was durch genügend kleine Wahl der Flächenelemente erreicht wird, und keine Winkelabhängigkeit zeigt (was bei der Voraussetzung $d \gg r_{\text {wd }}$ legitim ist),

$$
F_{\text {obs }}(\nu)=\frac{I\left(\theta_{\text {out }}, \nu\right) A_{\text {eff }}}{d^{2}} .
$$

Das Spektrum $S(\nu)$ des gesamten Sterns ergibt sich durch Summation über alle sichtbaren Flächenelemente

$$
S(\nu)=\sum_{\mathcal{A} \in \mathcal{V}} \frac{I\left(\theta_{\mathrm{out}}, \nu\right) A_{\mathrm{eff}}}{d^{2}} .
$$


Näherungsweise kann das Spektrum auch berechnet werden als gewichtete Summe aller ausgehenden Flüsse, die die jeweiligen Flächenelemente durchdringen. Da hier jedoch der winkelgemittelte Fluss benutzt wird, werden Randeffekte vernachlässigt. Weiterhin muss noch mit dem Faktor $\left(r_{\mathrm{wd}} / d\right)^{2}$ multipliziert werden, um die Verdünnung zu berücksichtigen

$$
\begin{aligned}
S(\nu) & =\left(\frac{r_{\mathrm{wd}}}{d}\right)^{2} \frac{1}{A_{\text {total }}} \sum_{\mathcal{A} \in \mathcal{V}} A_{\text {eff }} F_{\text {eff }} \\
& =\left(\frac{r_{\mathrm{wd}}}{d}\right)^{2} \frac{1}{A_{\text {total }}} \sum_{\mathcal{A} \in \mathcal{V}} \vec{A} \cdot \vec{n}_{\text {obs }} F_{\text {out }}(\nu, \mathcal{E}, \mathcal{A}) \cos \theta_{\text {out }} \\
& =\left(\frac{r_{\mathrm{wd}}}{d}\right)^{2} \frac{1}{A_{\text {total }}} \sum_{\mathcal{A} \in \mathcal{V}}|\vec{A}| F_{\text {out }}(\nu, \mathcal{E}, \mathcal{A}) \cos ^{2} \theta_{\text {out }}
\end{aligned}
$$

\section{$3.2 \quad$ Ergebnisse}

Wir zeigen einige Resultate von wd_model. Als Voraussetzung dient ein berechnetes Modellatmosphärengitter mit 10 Winkeln und verschiedenen Bestrahlungsflüssen. Die Parameter des Weißen Zwerges sind

$$
\begin{aligned}
T_{\text {eff }} & =20000 \mathrm{~K} \\
\log \left[\mathrm{g} /\left(\mathrm{cm} \mathrm{s}^{-2}\right)\right] & =8
\end{aligned}
$$

Wir betrachten das Modell unter dem Aspekt, inwiefern sich bei maximaler Sichtbarkeit der bestrahlten Fläche $\left(\theta_{\text {er }}=i=0, \varphi_{\text {er }}=0, \varphi_{\text {orb }}=0\right)$ das Spektrum mit zunehmender Bestrahlung verändert. Zunächst wird eine Emissionsregion gewählt, die aus einer einfachen isotrop strahlenden Punktquelle besteht. Danach folgen kreisförmige Emissionsregionen mit verschiedenen Radien.

\subsubsection{Punktquelle}

Die Punktquelle schwebt in einer Höhe von $h=0.1 r_{\text {wd }}$ über der Oberfläche des Weißen Zwerges. Es werden verschiedene Leuchtkräfte der Bestrahlung gewählt,

$$
\begin{aligned}
& L_{1}=1 \cdot 10^{31} \mathrm{erg} \mathrm{s}^{-1}, \\
& L_{2}=5 \cdot 10^{31} \mathrm{erg} \mathrm{s}^{-1}, \\
& L_{3}=1 \cdot 10^{32} \mathrm{erg} \mathrm{s}^{-1}, \\
& L_{4}=5 \cdot 10^{32} \mathrm{erg} \mathrm{s}^{-1} .
\end{aligned}
$$

Die Abbildungen 3.7 können als Flusskarten bezeichnet werden. Auf der Abszissenachse ist der Winkel $\alpha$ aufgetragen. $\alpha$ bezeichnet den Winkel zwischen dem Normalenvektor eines Flächenelements und der Verbindungsstrecke vom Zentrum des Sterns zum Zentrum der Emissionsregion (bei einer Punktquelle entspricht dies 
der Position der Quelle, bei einer kreisförmigen Emissionsregion entspricht es dem Mittelpunkt der Kreisfläche). Auf der Ordinatenachse ist der Bestrahlungsfluss aufgetragen. Die gestrichelten Linien repräsentieren den theoretisch berechneten einfallenden Fluss, der bei einem Flächenelement mit dem Winkel $\alpha$ ankommt. Die dreieckig markierten Punkte stellen die aus dem Modellatmosphärengitter verwendeten Modelle dar. Idealerweise sollten alle benutzten Modellatmosphären auf der gestrichelten Linie liegen, d.h. dem theoretischen Fluss entsprechen. Es lässt sich erkennen, dass dies für höhere Bestrahlungsleuchtkräfte (siehe $L=5 \cdot 10^{32} \mathrm{erg} \mathrm{s}^{-1}$ in Abb. 3.7) nicht mehr erreicht werden kann, da der bei den entsprechenden Flächenelementen vorhandene Bestrahlungsfluss $F_{\text {in }}$ über der kritischen Grenze liegt, welche mit dem Atmosphärenprogramm atX berechnet werden kann (siehe 2.2.1).

Abbildung 3.8 zeigt die resultierenden Spektren des bestrahlten Weißen Zwerges im Bereich der Ly $\alpha$ Linie. Ein Kurve der Schar bezieht sich auf eine bestimmte Bestrahlungsleuchtkraft $\left(L_{1}-L_{4}\right.$ von unten nach oben). Es ist ersichtlich, dass selbst im Fall der stärksten Leuchtkräfte, die schon nicht mehr durch das Gitter der Modellatmosphären abgedeckt werden können, die Bestrahlung nicht ausreicht, um die Ly $\alpha$ Absorptionslinie in den Flügeln wesentlich zu modifizieren. Lediglich der schmale Linienkern entwickelt Emission aufgrund der Temperaturinversion der äußeren Schichten. 

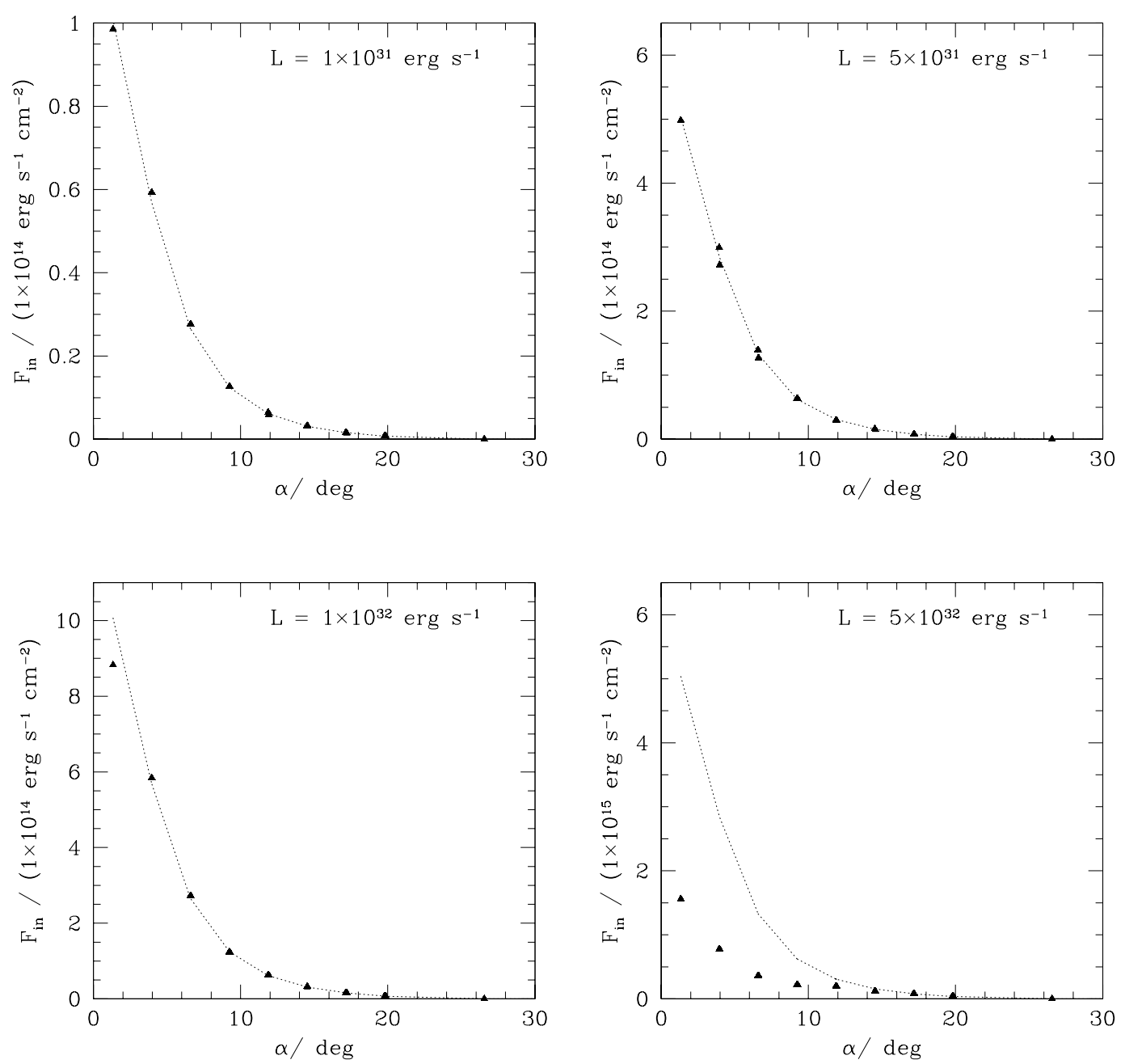

Abbildung 3.7: Zuordnung der Modellatmosphären zu den Flächenelementen. Auf der Abszisse ist der Winkel $\alpha$ aufgetragen. Dieser entspricht dem Winkel zwischen Flächenelementnormale und Positionsvektor der Punktquelle. Auf der Ordinate befindet sich der Bestrahlungsfluss $F_{\text {in }}$. Die gestrichelte Linie stellt den theoretischen Bestrahlungsfluss dar, der bei gegebener Emissionsregion bei einem Flächenelement ankommt. Die Punkte entsprechen dem aus dem Modellgitter selektierten Fluss. Das letzte Modell mit $L=5 \cdot 10^{32} \mathrm{erg} \mathrm{s}^{-1} \mathrm{kann}$ mit den in der Datenbank vorhandenen Modellen nicht mehr abgedeckt werden. 


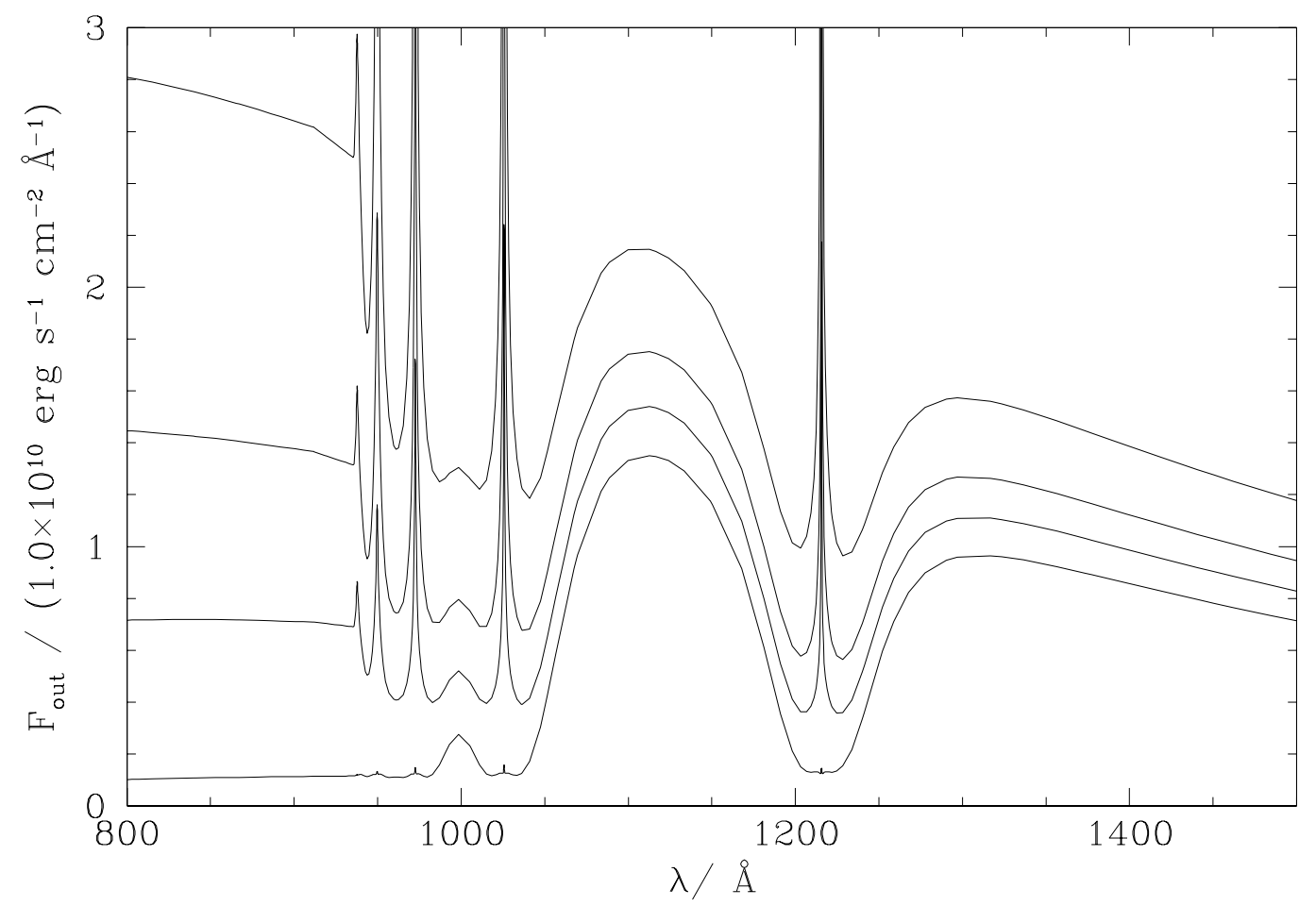

Abbildung 3.8: Das Gesamtspektrum in der Lyman-Region für einen durch eine Punktquelle in der Höhe $h=0.1 r_{\text {wd }}$ bestrahlten Weißen Zwerg. Die Phase beträgt $\varphi_{\text {orb }}=0$, d.h. das Zentrum des gehitzten Flecks ist dem Beobachter zugewandt. Die Kurven der Schar beziehen sich jeweils auf eine Bestrahlungsleuchtkraft $L$ (von unten nach oben: $L_{1}=1 \cdot 10^{31} \mathrm{erg} \mathrm{s}^{-1}, L_{2}=5 \cdot 10^{31} \mathrm{erg} \mathrm{s}^{-1}, L_{3}=1 \cdot 10^{32} \mathrm{erg} \mathrm{s}^{-1}$ und $\left.L_{4}=5 \cdot 10^{32} \mathrm{erg} \mathrm{s}^{-1}\right)$. 


\subsubsection{Kreisförmige Emissionsregion}

Wir erweitern nun die Form der Emissionsregion auf eine kreisförmige Region, die jedoch weiterhin aus isotrop strahlenden Punktquellen zusammengesetzt wird. Motiviert wird dieser Ansatz durch den Wunsch, eine größere Fläche der Oberfläche des Weißen Zwerges unter einem noch relativ steilen Winkel zu bestrahlen. Es hatte sich gezeigt, dass im Rahmen unserer Modellatmosphären die Bestrahlung mit einer isotropen Punktquelle nicht ausreichend ist, um eine signifikante Auffüllung der Linienflügel zu erreichen.

Wir zeigen nun Modelle mit kreisförmigen Emissionsregionen mit den Radien

$$
\begin{aligned}
& r_{1}=0.1 r_{\mathrm{wd}}, \\
& r_{2}=0.2 r_{\mathrm{wd}}, \\
& r_{3}=0.3 r_{\mathrm{wd}},
\end{aligned}
$$

die in einer Höhe von

$$
h=0.1 r_{\mathrm{wd}}
$$

über der Oberfläche des Weißen Zwerges schweben.

Eine kreisförmige Emissionsregion ist aus Punktquellen zusammengesetzt. Das prinzipielle Problem, dass hierbei Einstrahlung unter verschiedenen Winkeln stattfindet, die mit den vorhandenen Modellatmosphären nicht behandelt werden kann, wird durch den in Gl. 3.22 definierten gemittelten Bestrahlungswinkel approximativ gelöst. Abbildung 3.9 zeigt den Vergleich der gemittelten Bestrahlungswinkel. Auf der Abszisse ist der Winkel $\alpha$ zwischen dem Normalenvektor eines Flächenelementes und dem Normalenvektor der Emissionsregion aufgetragen. Auf der Ordinate findet man den entsprechenden gemittelten Bestrahlungswinkel, wobei $\theta_{\text {in }}=0$ senkrechter Einstrahlung entspricht. Wie erwartet vergrößert sich die Fläche der Atmosphäre, die noch unter einem relativ steilen Winkel $\theta_{\text {in }} \lesssim 50^{\circ}$ bestrahlt wird.

Die Bestrahlungsleuchtkräfte betragen

$$
\begin{aligned}
& L_{1}=1 \cdot 10^{31} \mathrm{erg} \mathrm{s}^{-1}, \\
& L_{2}=5 \cdot 10^{31} \mathrm{erg} \mathrm{s}^{-1}, \\
& L_{3}=1 \cdot 10^{32} \mathrm{erg} \mathrm{s}^{-1}, \\
& L_{4}=5 \cdot 10^{32} \mathrm{erg} \mathrm{s}^{-1} .
\end{aligned}
$$

Abbildungen 3.11, 3.13, 3.15 zeigen die Zuordnung der Bestrahlungsflüsse der Atmosphärenmodelle zu den Flächenelementen für jeweils eine vorgegebene Leuchtkraft der Emissionsregion. Die Modelle mit der Leuchtkraft $L<5 \cdot 10^{32} \mathrm{erg} \mathrm{s}^{-1}$ können mit den vorhandenen Atmosphärenmodellen gut abgedeckt werden. Der Fall $L=5 \cdot 10^{32} \mathrm{erg} \mathrm{s}^{-1}$ kann nicht mehr einwandfrei behandelt werden. Die Abbildungen 3.12, 3.14 und 3.16 zeigen die resultierenden Spektren des bestrahlten Sterns in der Lyman-Region von $\lambda=800-1500 \AA$. In einem Diagramm sind jeweils die vier verschiedenen Leuchtkräfte (3.39) von unten nach oben mit zunehmendem $L$ dargestellt. 


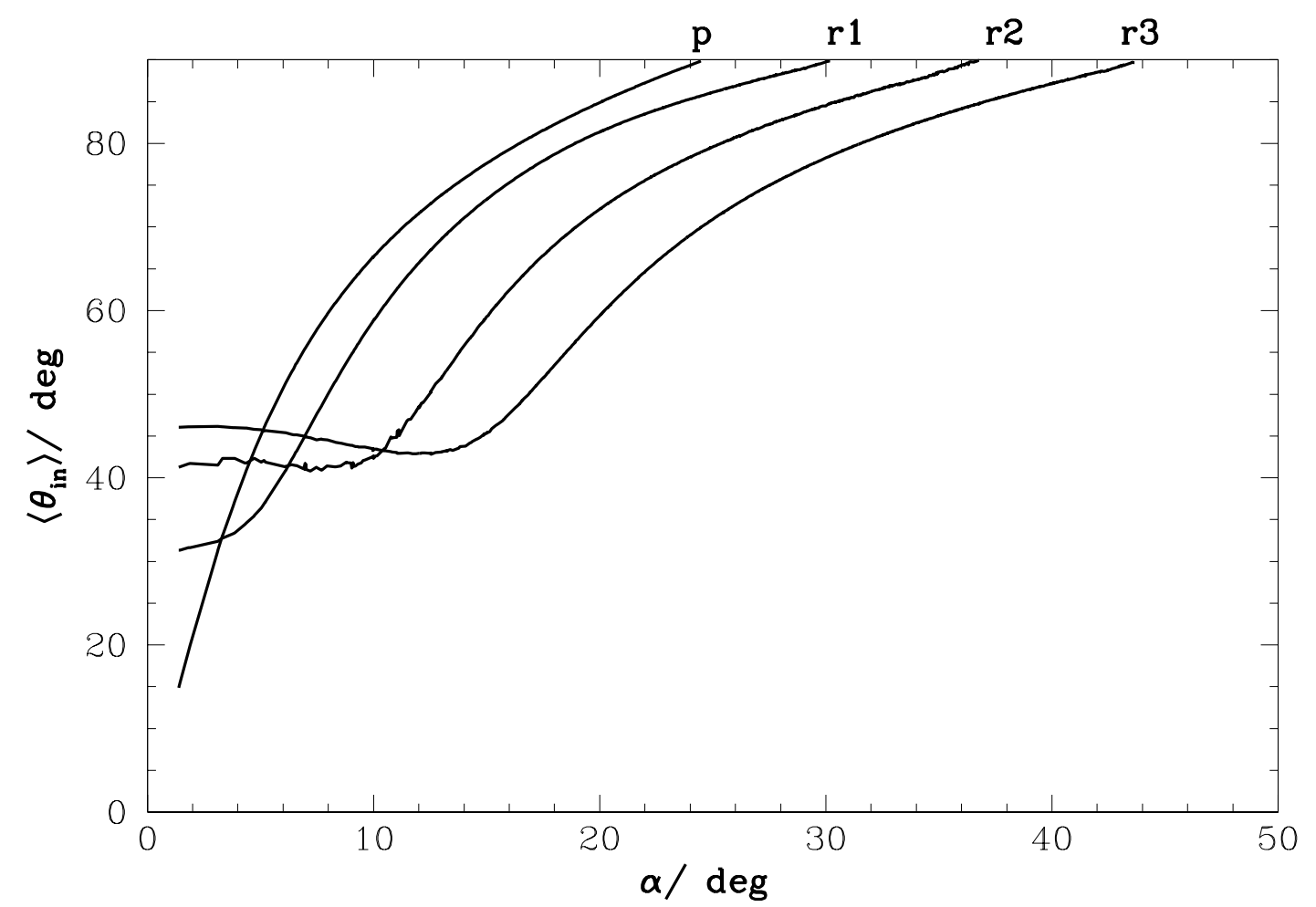

Abbildung 3.9: Darstellung des nach Gl. 3.22 gemittelten Bestrahlungswinkel $\theta_{\text {in }}$ für verschiedene Emissionsregionen. Auf der Abszisse ist der Winkel $\alpha$ aufgetragen. Auf der Ordinate befindet sich $\theta_{\text {in }}$. Die mit $\mathrm{p}$ markierte Kurve repräsentiert die Verteilung für eine Punktquelle. Die mit $r 1$, r2 und r3 bezeichneten Kurven stellen die Verteilung für kreisförmige Emissionsregionen mit den Radien 0.1,0.2 und 0.3 in Einheiten von $r_{\mathrm{wd}}$ dar. 

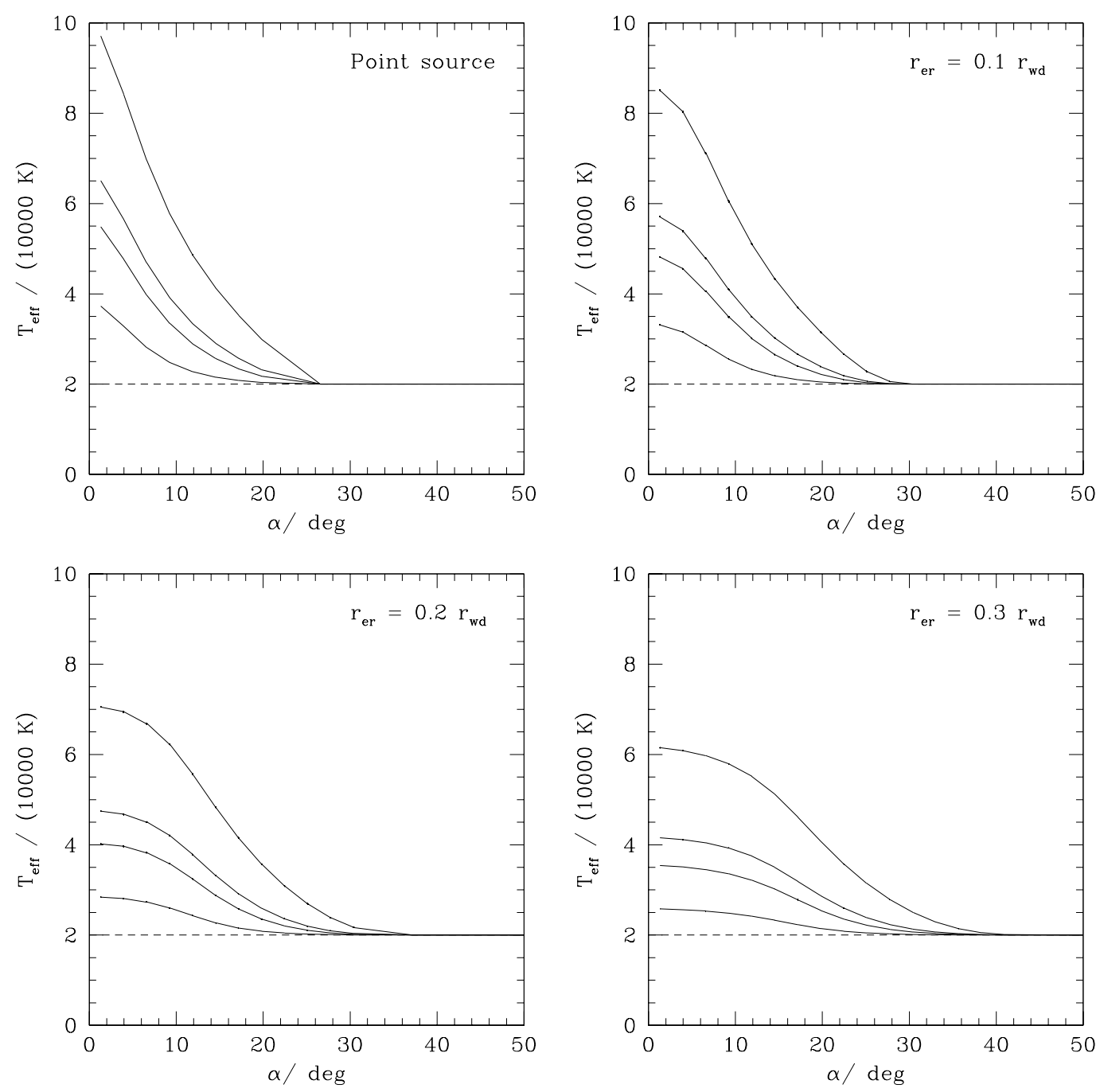

Abbildung 3.10: Die Temperaturen der Flächenelemente, die sich aus der gegebenen Effektivtemperatur $T_{\text {eff }}=20000$ des Weißen Zwerges (gestrichelt) und dem jeweiligen Bestrahlungsfluss $F_{\text {in }}$ berechnen, aufgetragen in Abhängigkeit vom Winkel $\alpha$. Die einzelnen Kurven der Schar in einem Diagramm gehören zu einer definierten Bestrahlungsleuchtkraft. Von unten nach oben $L_{1}=1 \cdot 10^{31} \mathrm{erg} \mathrm{s}^{-1}, L_{2}=5 \cdot 10^{31} \mathrm{erg} \mathrm{s}^{-1}$, $L_{3}=1 \cdot 10^{32} \mathrm{erg} \mathrm{s}^{-1}$ und $L_{4}=5 \cdot 10^{32} \mathrm{erg} \mathrm{s}^{-1}$. Die einzelnen Abbildungen repräsentieren die Verteilungen für verschiedene Geometrien der Emissionsregion. Oben links: Punktquelle, oben rechts: Kreisförmige Emissionsregion mit $r_{\mathrm{er}}=0.1 r_{\mathrm{wd}}$, unten links: $r_{\mathrm{er}}=0.2 r_{\mathrm{wd}}$ und unten rechts: $r_{\mathrm{er}}=0.3 r_{\mathrm{wd}}$. 
Kreisförmige Emissionsregion mit einem Radius $r_{\mathrm{er}}=0.1 \cdot r_{\mathrm{wd}}$
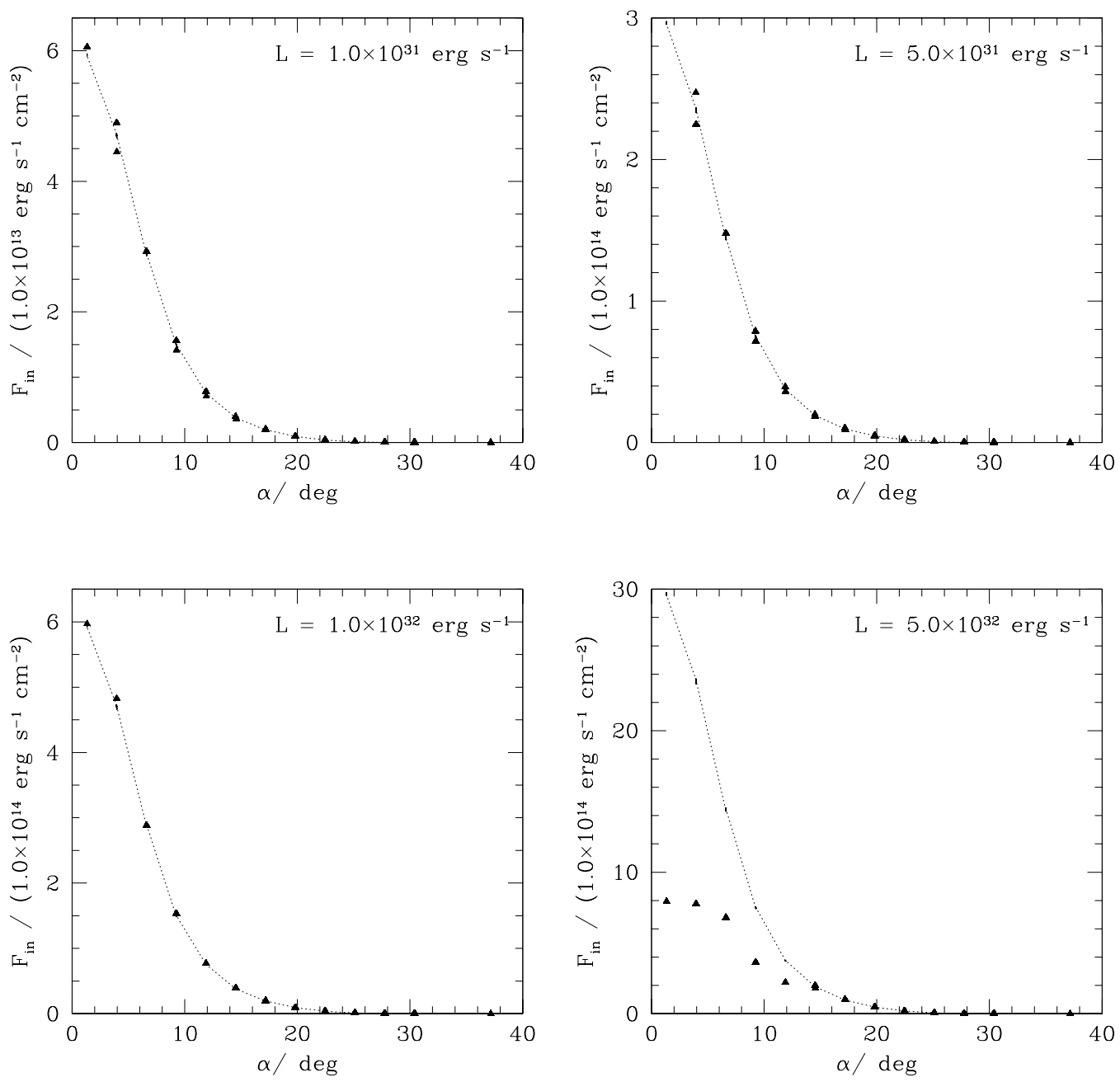

Abbildung 3.11: Analog zur Abb. 3.7 für die Punktquelle sieht man hier die Zuordnung der Bestrahlungsflüsse $F_{\text {in }} \mathrm{zu}$ den Flächenelementen für eine ausgedehnte kreisförmige Emissionsregion mit dem Radius $r_{\mathrm{er}}=0.1 r_{\mathrm{wd}}$. Auf der Abszisse ist der Winkel zwischen Flächenelementnormale und Emissionsregionnormale aufgetragen. Auf der Ordinate befindet sich der Bestrahlungsfluss $F_{\text {in }}$. Die Linien zeigen die theoretischen Werte, die Dreiecke stellen die im Gitter vorhandenen Atmosphärenmodelle dar. 


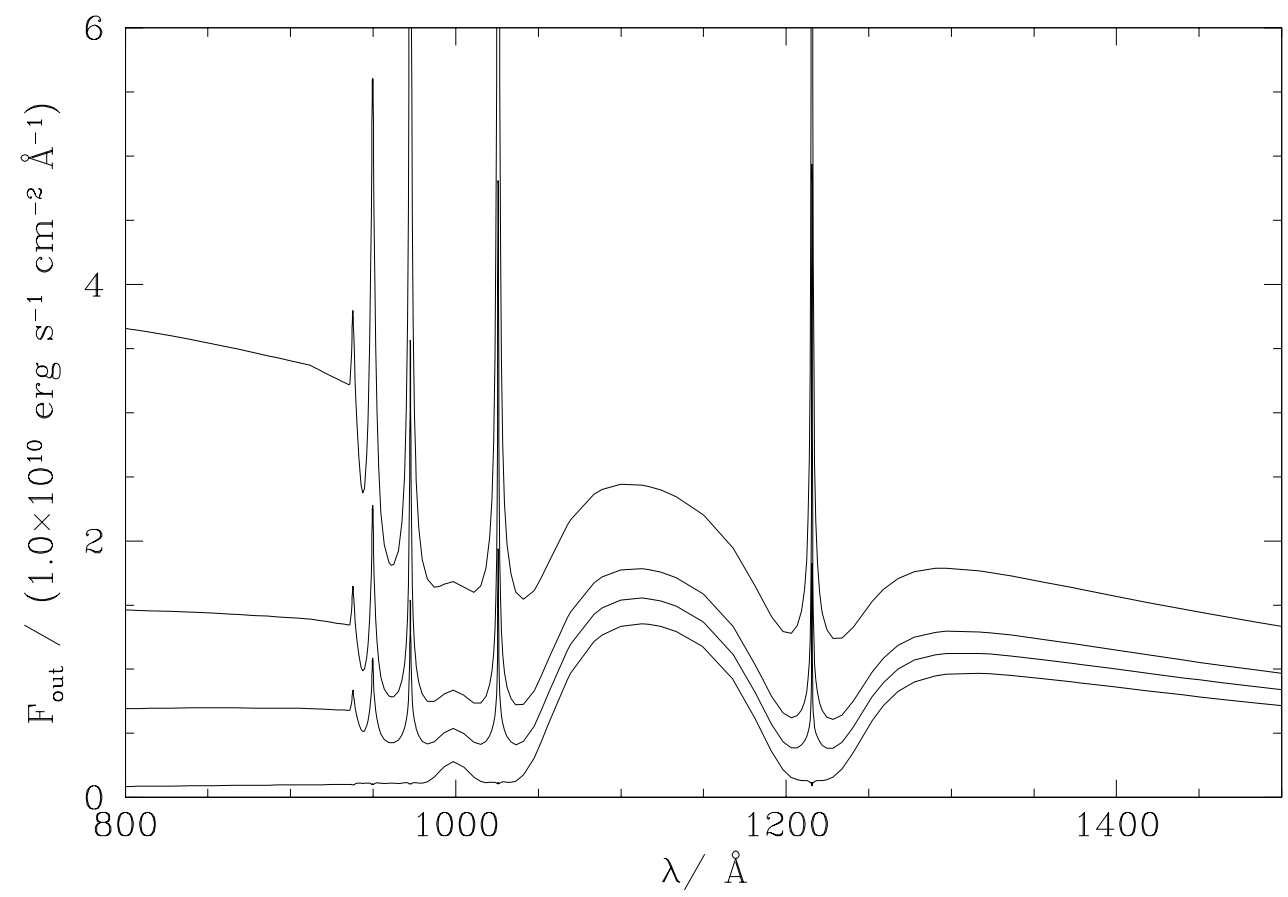

Abbildung 3.12: Das Gesamtspektrum des mit einer kreisförmigen Emissionsregion mit dem Radius $r_{\mathrm{er}}=0.1 r_{\mathrm{wd}}$ bestrahlten Weißen Zwerges in der Lyman-Region $800 \AA \leq \lambda \leq 1500 \AA$. Die Phase beträgt $\varphi_{\text {orb }}=0$. Eine Kurve der Schar bezieht sich auf eine bestimmte Leuchtkraft $L$ (von unten nach oben $L_{1}=1 \cdot 10^{31} \mathrm{erg} \mathrm{s}^{-1}$, $L_{2}=5 \cdot 10^{31} \mathrm{erg} \mathrm{s}^{-1}, L_{3}=1 \cdot 10^{32} \mathrm{erg} \mathrm{s}^{-1}$ und $\left.L_{4}=5 \cdot 10^{32} \mathrm{erg} \mathrm{s}^{-1}\right)$. 
Kreisförmige Emissionsregion mit einem Radius $r_{\mathrm{er}}=0.2 \cdot r_{\mathrm{wd}}$
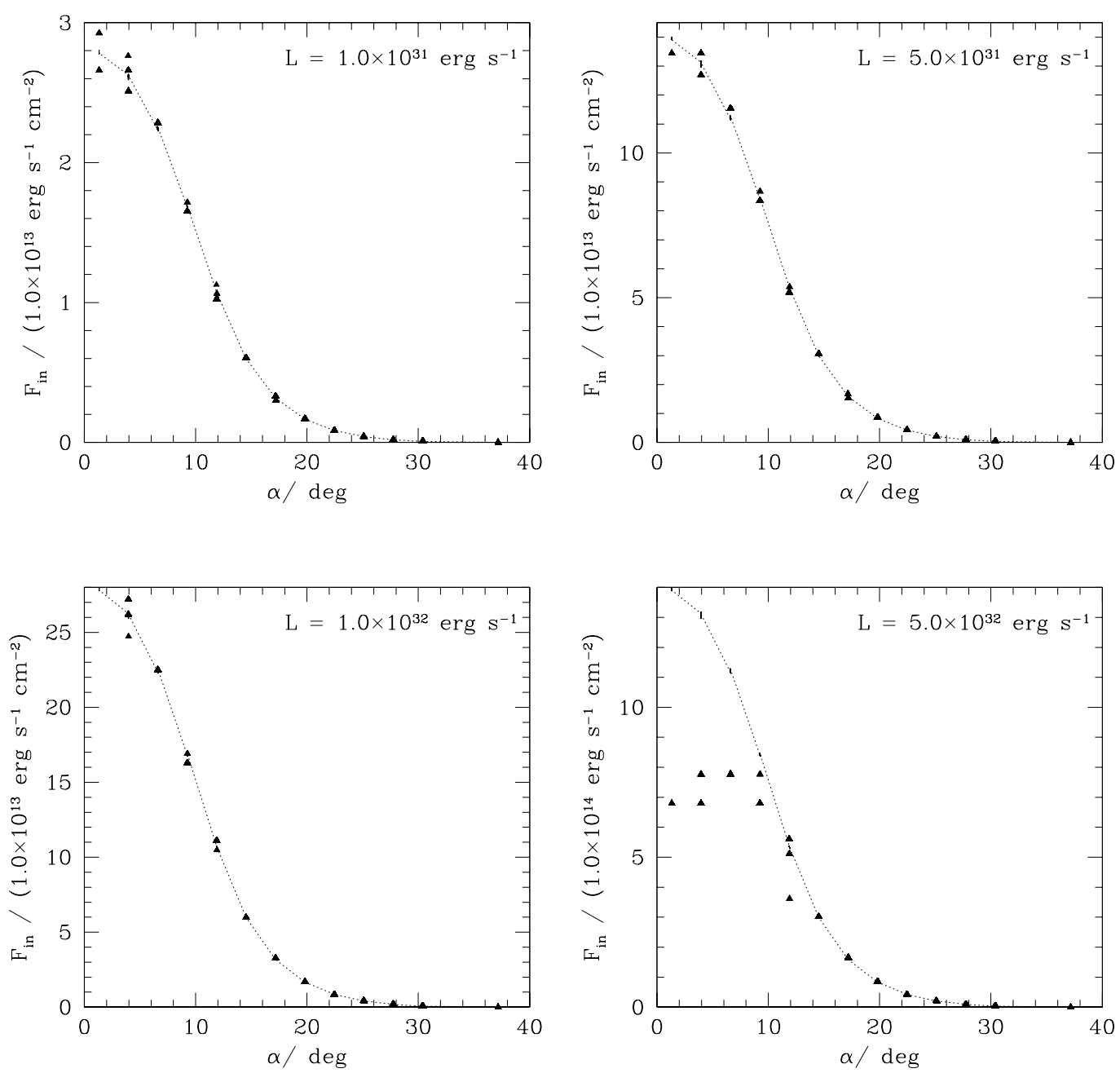

Abbildung 3.13: Analog zur Abb. 3.11 sieht man hier die Zuordnung der Bestrahlungsflüsse $F_{\text {in }}$ zu den Flächenelementen für eine ausgedehnte kreisförmige Emissionsregion mit dem Radius $r_{\mathrm{er}}=0.2 r_{\mathrm{wd}}$. Erläuterung siehe Abb. 3.11. 


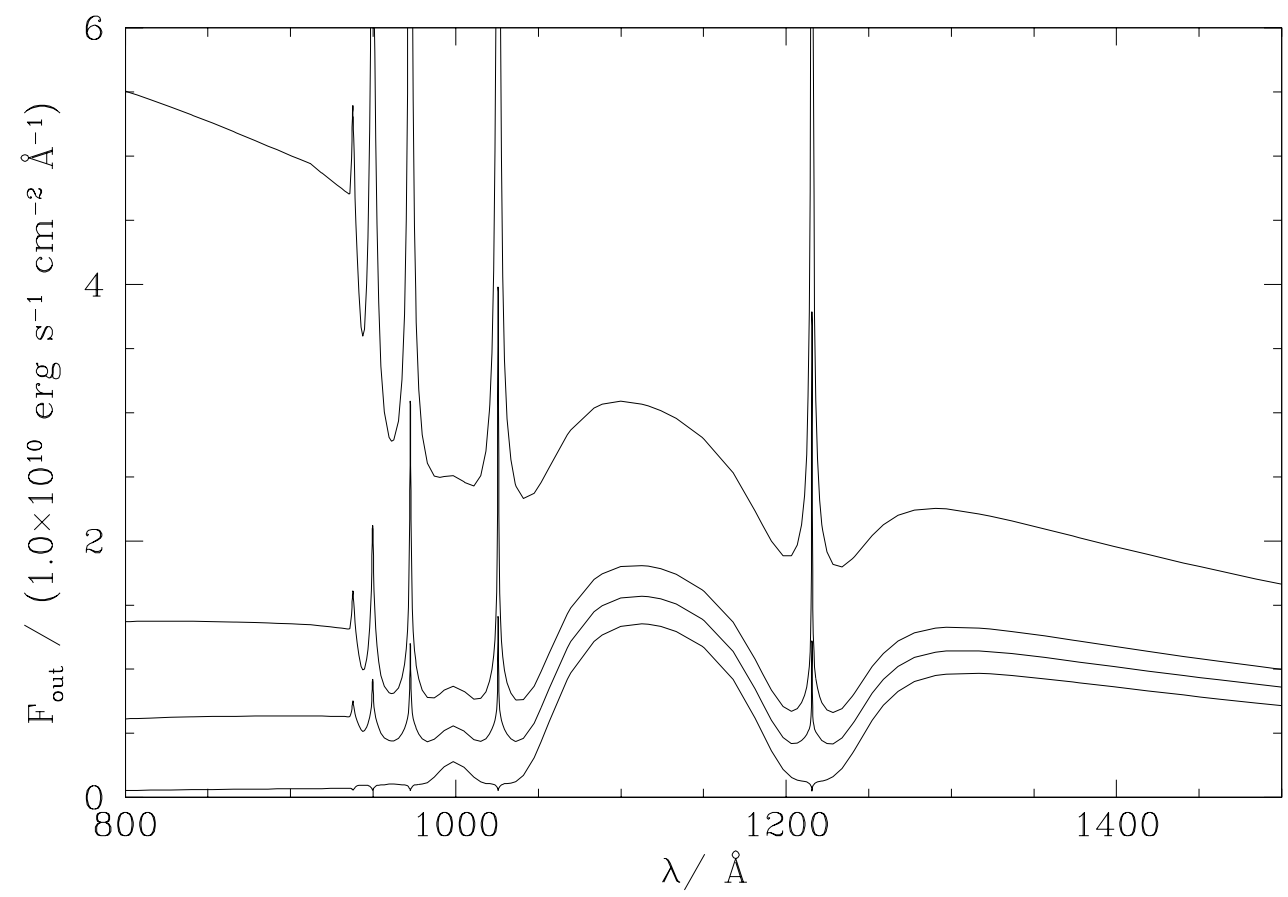

Abbildung 3.14: Gesamtspektrum des bestrahlten Weißen Zwerges in der LymanRegion. Der Radius der kreisförmigen Emissionsregion beträgt $r_{\mathrm{er}}=0.2 r_{\mathrm{wd}}$. Die Phase beträgt $\varphi_{\text {orb }}=0$. Eine Kurve der Schar bezieht sich auf eine bestimmte Leuchtkraft $L$ (von unten nach oben $L_{1}=1 \cdot 10^{31} \mathrm{erg} \mathrm{s}^{-1}, L_{2}=5 \cdot 10^{31} \mathrm{erg} \mathrm{s}^{-1}$, $L_{3}=1 \cdot 10^{32} \mathrm{erg} \mathrm{s}^{-1}$ und $\left.L_{4}=5 \cdot 10^{32} \mathrm{erg} \mathrm{s}^{-1}\right)$. 
Kreisförmige Emissionsregion mit einem Radius $r_{\mathrm{er}}=0.3 \cdot r_{\mathrm{wd}}$
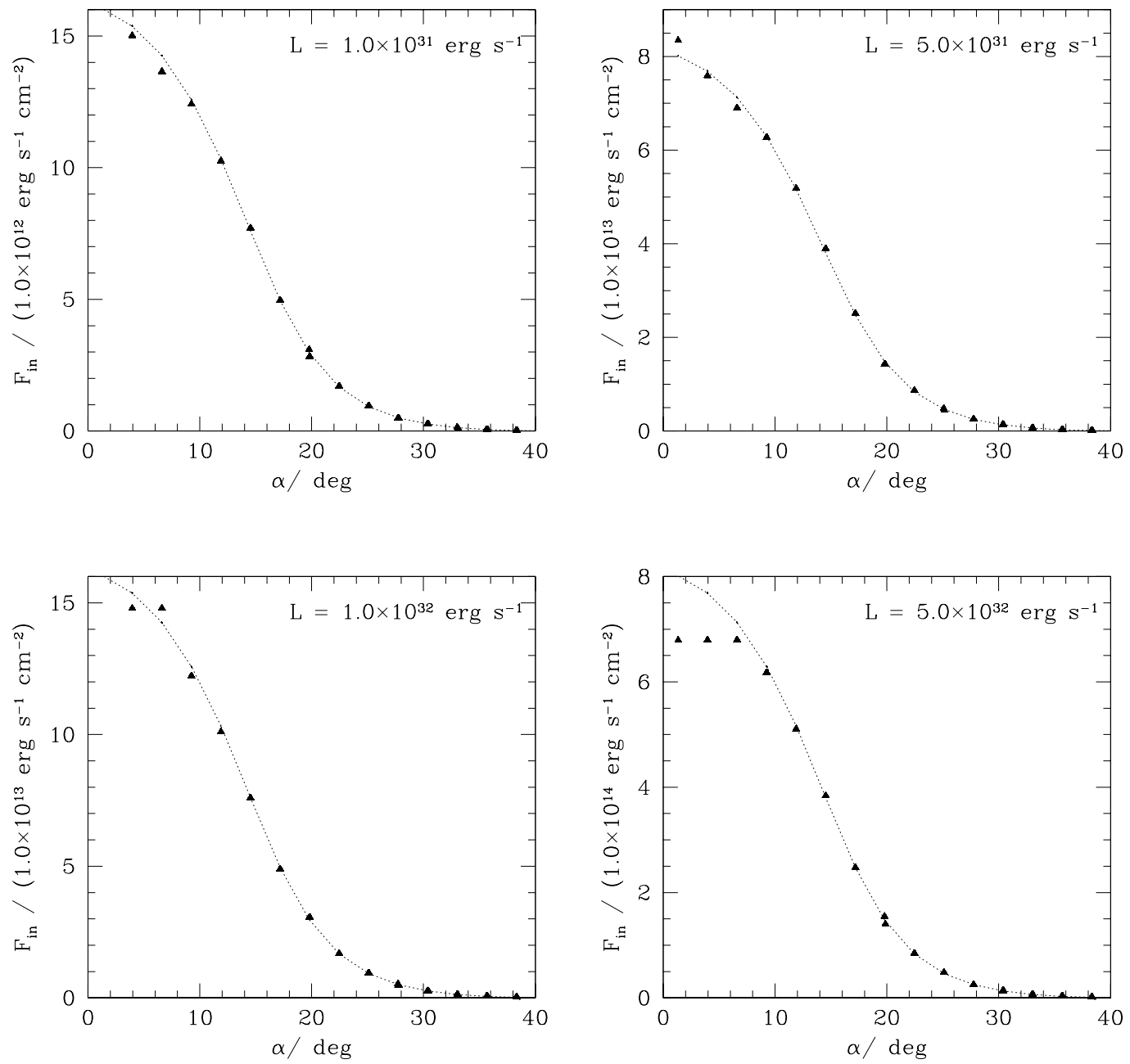

Abbildung 3.15: Analog zur Abb. 3.11 und Abb. 3.13 sieht man hier die Zuordnung der Bestrahlungsflüsse $F_{\text {in }}$ zu den Flächenelementen für eine ausgedehnte kreisförmige Emissionsregion mit dem Radius $r_{\mathrm{er}}=0.3 r_{\mathrm{wd}}$. Erläuterung siehe Abb. 3.11 . 


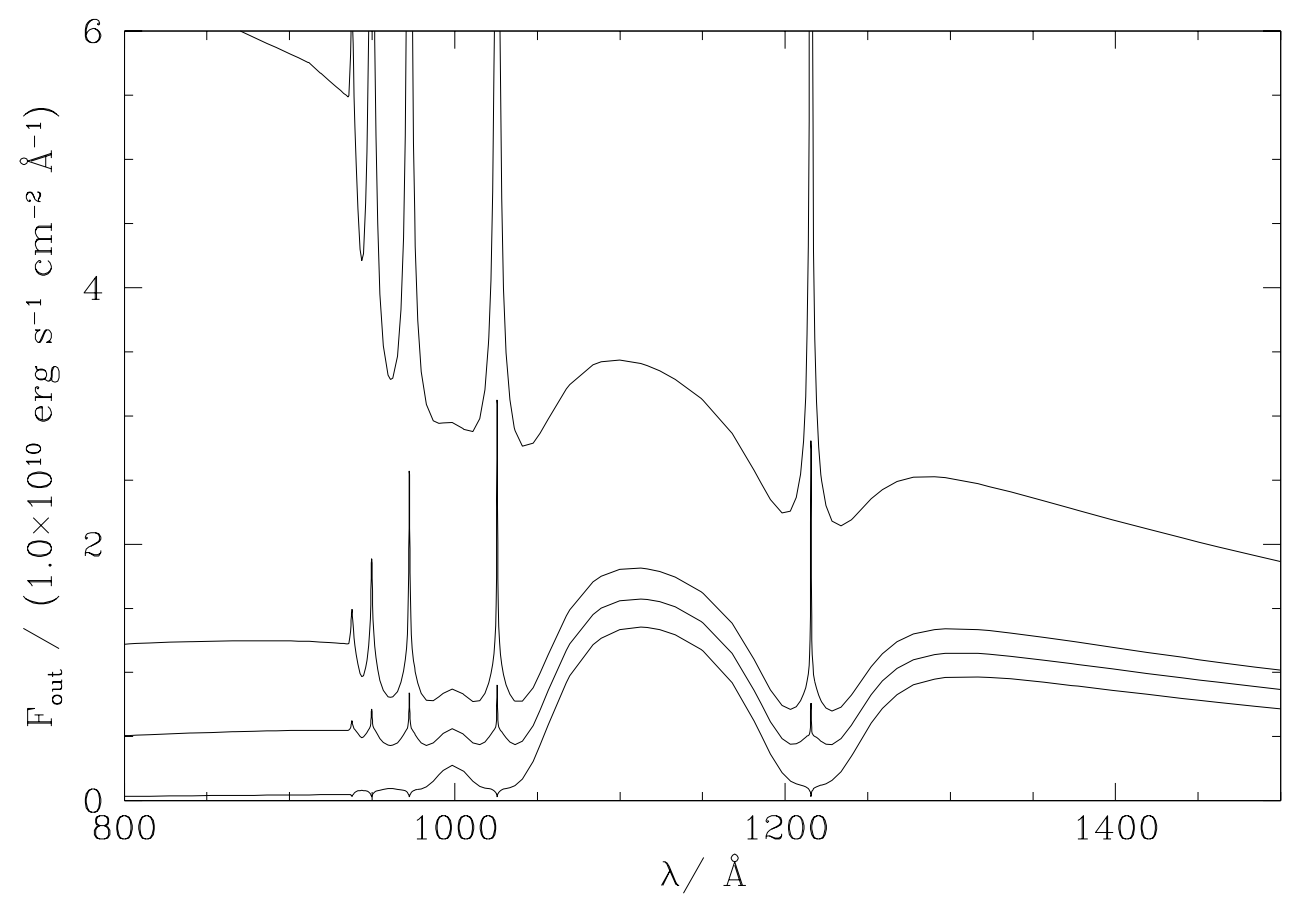

Abbildung 3.16: Gesamtspektrum des Weißen Zwerges, der mit einer kreisförmigen Emissionsregion mit dem Radius $r_{\text {er }}=0.3 r_{\text {wd }}$ bestrahlt wird. Die Phase beträgt $\varphi_{\text {orb }}=0$. Man sieht im Vergleich zu den Abbildungen 3.12 und 3.14, dass die Auffüllung der Ly $\alpha$ Linie stärker ist. 


\section{Kapitel 4}

\section{Anwendung auf Beobachtungen von AM Her}

\subsection{Modellierung}

Wir wenden nun die Ergebnisse des dreidimensionalen Modells auf die Beobachtungen des Polars AM Her an. Wie wir in Kapitel 1.3 gesehen haben, dominiert im UVBereich die Strahlung des Weißen Zwerges. Diese zeigt dort eine phasenabhängige quasisinusförmige Modulation, die durch die Rotation im Zusammenhang mit dem heißen Fleck auf der Oberfläche des Weißen Zwerges erzeugt wird. Daneben zeigt sich eine signifikante Auffüllung der Ly $\alpha$-Absorptionslinie im Spektrum.

In den Modellrechnungen aus Abschnitt 3.2 kann man erkennen, dass die Bestrahlung in der Tat zu einem Verschwinden der Absorptionslinie führt. Allerdings führt eine Bestrahlung in dem Maße, wie sie benötigt wird, um die Linienflügel signifikant zu modifizieren, zu deutlichen Emissionslinien im Kern der Linie. Dieses Problem wird noch zu diskutieren sein, denn einerseits fehlen in den Beobachtungen von AM Her sehr deutlich die Linienflügel der Ly $\alpha$-Linie, was eine Erklärung durch eine intensive Bestrahlung nahelegt, andererseits gibt es keinerlei Hinweise auf beobachtete Emission im Linienkern, soweit diese überhaupt zu erkennen ist, da die Linienkerne der Ly $\alpha$-Linie teilweise kontaminiert sind durch geokoronale Emission.

Hierzu ist von Bedeutung, dass der Kern der Linien der Lymanserie interstellarer Absorption unterliegt, d.h. der schmale Kern der Linien ist prinizpiell nicht zuverlässig beobachtbar. Somit ist zweifelhaft, ob die vorhandenen Emissionskerne der Modellspektren bei massiven Bestrahlungsstärken, die zu einer Temperaturinversion führen, überhaupt der Beobachtung zugänglich sind. Die interstellare Absorption läßt sich folgendermaßen modellieren: Die Säulendichte von Wasserstoff wurde in Gänsicke et al. (1998) bestimmt mit $N_{\mathrm{H}}=(3 \pm 1.5) \cdot 10^{19} \mathrm{~cm}^{-2}$. Bei ausschließlicher Berücksichtigung von Strahlungsdämpfung ist das entsprechende Absorptionsprofil gegeben durch (Bohlin, 1973)

$$
f(\lambda)=e^{-\tau(\lambda)}
$$




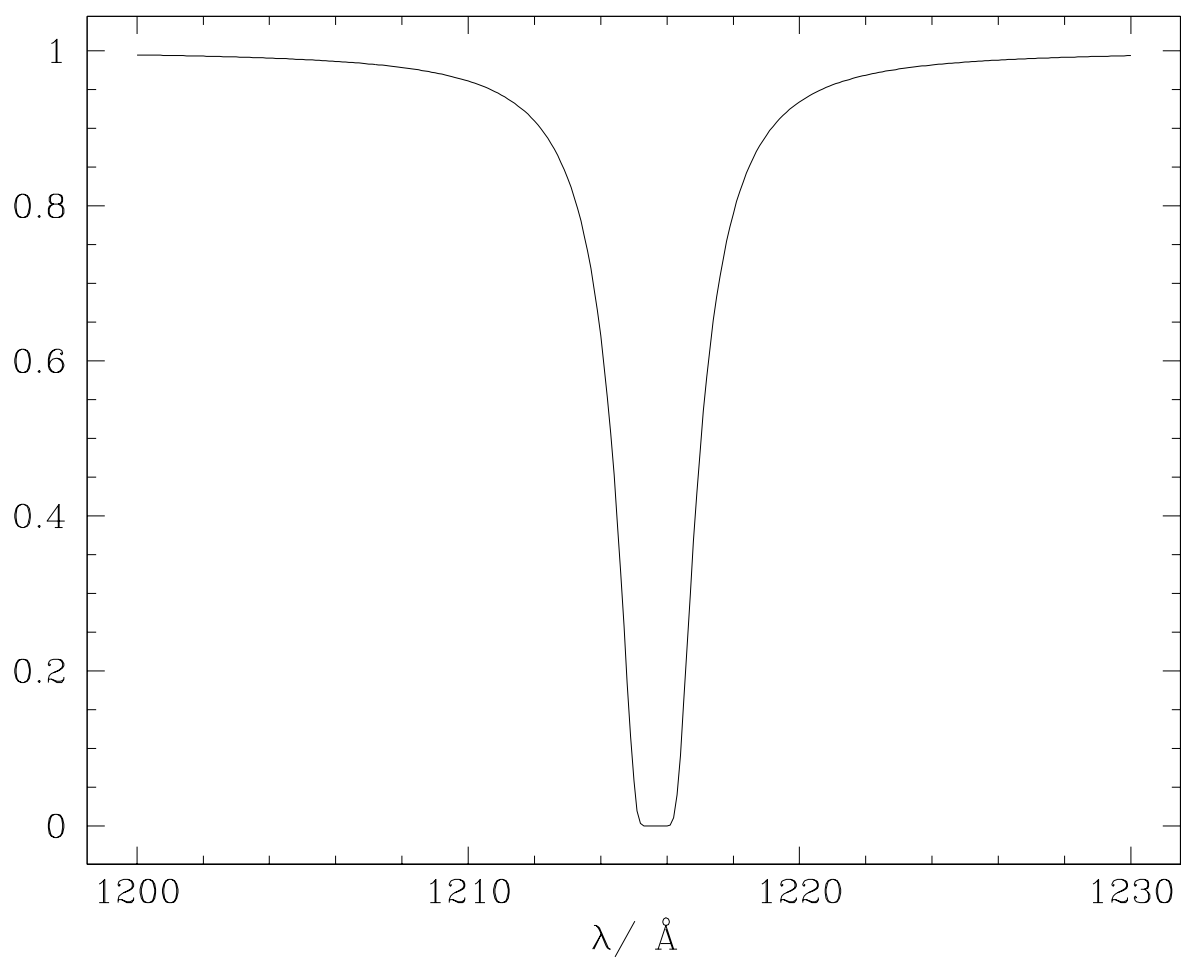

Abbildung 4.1: Das Absorptionsprofil $f(\lambda)$ für die interstellare Ly $\alpha$-Absorption bei einer Wasserstoffsäulendichte von $N_{\mathrm{H}}=3 \cdot 10^{19} \mathrm{~cm}^{-2}$.

mit

$$
\tau(\lambda)=7.06 \cdot 10^{-11} \cdot N_{\mathrm{H}}\left[1+1.66 \cdot 10^{9}\left(\lambda-\lambda_{0}\right)^{2}\right] .
$$

Der mit dem interstellaren Medium wechselwirkende Fluß berechnet sich durch

$$
F(\lambda)=F_{0}(\lambda) \cdot f(\lambda)
$$

Die Funktion $f(\lambda)$ ist in Abbildung 4.1 dargestellt. Die dünnen Emissionskerne verschwinden vollständig. Lediglich in den breiteren Flügeln ist bei sehr starker Bestrahlung noch Emission zu erkennen. Da dieser Bereich in den Beobachtungen teilweise durch Emission aus dem Akkretionsstrom kontaminiert ist, lassen sich hierüber keine genauen Aussagen treffen.

\subsubsection{Unabhängige Modellierung der Phase $\varphi_{\text {orb }}=0.1$}

Wir versuchen, die Phase $\varphi_{\text {orb }}=0.1$ der verfügbaren HST/GHRS-Beobachtungen zu modellieren, zu der der heiße Fleck seine maximale geometrische Projektion erreicht. Dies geschieht zunächst unabhängig von anderen verfügbaren Daten, um 


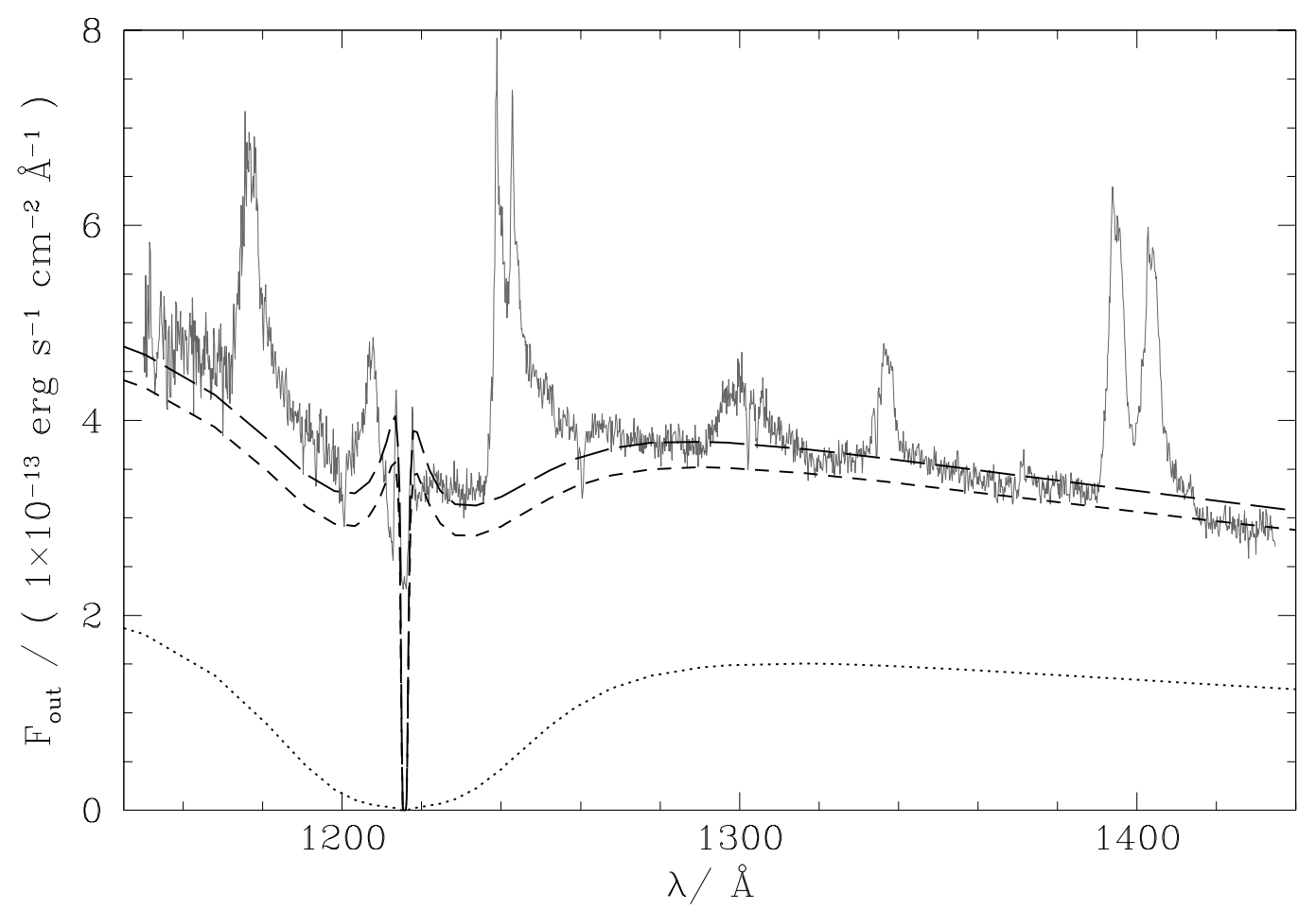

Abbildung 4.2: HST/GHRS Beobachtung zur Orbitalphase $\varphi_{\text {orb }}$ im Vergleich mit einem Modell mit den Parametern $r_{\mathrm{wd}}=1.12 \cdot 10^{9} \mathrm{~cm}, d=90 \mathrm{pc}, i=50^{\circ}, \theta_{\mathrm{er}}=50^{\circ}$, $h_{\mathrm{er}}=0.1 r_{\mathrm{wd}}, r_{\mathrm{er}}=0.3 r_{\mathrm{wd}}$ Die kurz gestrichelte Kurve entspricht dem Spektrum des Modells mit einer Leuchtkraft von $L=6 \cdot 10^{32} \mathrm{erg} \mathrm{s}^{-1}$, die lang gestrichelte $L=7 \cdot 10^{32} \mathrm{erg} \mathrm{s}^{-1}$. Das Spektrum eines unbestrahlten Weißen Zwerges ist durch die gepunktete Kurve dargestellt.

zu sehen, ob sich die Auffüllung der Ly $\alpha$-Linie prinzipiell mit den für AM Her typischen Parametern rekonstruieren lässt. In diesem Abschnitt wird davon ausgegangen, dass die gesamte im betrachteten Bereich $\lambda=1150-1450 \AA$ vorhandene Strahlung ihren Ursprung in der Atmosphäre des Weißen Zwerges besitzt. In Abbildung 4.2 ist ein Modell im Vergleich mit den Beobachtungsdaten abgebildet. Die betrachtete Orbitalphase ist $\varphi_{\text {orb }}=0.1$. Der Systemabstand zum Beobachter wurde mit $d=90 \mathrm{pc}$ angenommen (Gänsicke et al., 1995). Der Radius des Weißen Zwerges beträgt $r_{\mathrm{wd}}=1.12 \cdot 10^{9} \mathrm{~cm}$. Die Inklination des Systems beträgt $i=50^{\circ}$ und die Komplementbreite (Winkel zwischen Zentrum der Emissionsregion und der Rotationsachse) beträgt $\theta_{\mathrm{er}}=50^{\circ}$ (Gänsicke et al., 2001). Das Modell besitzt eine kreisförmige Emissionsregion mit $r_{\mathrm{er}}=0.3 r_{\mathrm{wd}}$. Die Höhe der Emissionsregion beträgt $h=0.1 r_{\mathrm{wd}}$. Die Systemparameter sind angelehnt an Gänsicke et al. (1998), allerdings muss darauf hingewiesen werden, dass diese Modellierung nicht konsistent 


\begin{tabular}{ccc}
\hline & $F(1300 \AA) /\left(10^{-13} \mathrm{ergs}^{-1} \mathrm{~cm}^{-2} \AA^{-1}\right)$ \\
& Max. Phase & Min. Phase \\
\hline Lo & 1.3 & 0.8 \\
Hi & 3.7 & 1.5 \\
\hline
\end{tabular}

Tabelle 4.1: Absolute Flüsse $F(\lambda)$ bei $\lambda=1300 \AA$ von AM Her aus den IUE-Daten im Low und High State zu jeweils minimaler und maximaler Phase (Gänsicke et al., 1995).

ist mit entsprechenden Beobachtungen im Zustand niedriger Akkretionsrate (Low State) des Systems. Der Fluß des unbestrahlten Weißen Zwerges z.B. bei $\lambda=1300 \AA$ ist um etwa einen Faktor zwei höher als die entsprechenden Low State Daten zeigen. Diese Modellierung geht davon aus, dass der gesamte im betrachteten Intervall vohandene Strahlungsfluss der Atmosphäre des Weißen Zwerges entspringt, was - wie im nachfolgenden Abschnitt diskutiert wird - nicht realistisch ist. Das Modell zeigt jedoch, dass das beobachtete Spektrum zur maximalen Phase adäquat beschrieben werden kann. Die Auffüllung der Ly $\alpha$-Linie zentriert um $\lambda=1216 \AA$ zeigt eine akzeptable Übereinstimmung.

\subsubsection{Konsistente Modellierung mehrerer Phasen}

Es folgt nun der Versuch, mehrere beobachtete Spektren zu verschiedenen Phasen mit einem Modell zu erklären. Dabei soll außerdem berücksichtigt werden, dass die Modellierung mit den IUE-Daten von AM Her (Gänsicke et al., 1995), bei denen Low und High State-Daten verfügbar sind, und den HST/GHRS High-State Daten (Gänsicke et al., 1998) konsistent ist.

Das prinzipielle Problem wird sichtbar, wenn man sich die absoluten Flüsse $F(\lambda)$ des System bei der Wellenlänge $\lambda=1300 \AA \mathrm{zu}$ verschiedenen Zeitpunkten anschaut (Tabelle 4.1). Der Fluß im Low State zu minimaler Phase ist konsistent mit einem Modell eines Weißen Zwerges mit einer Effektivtemperatur $T_{\text {eff }}=20000 \mathrm{~K}$ und einem Radius $r_{\mathrm{wd}}=8.1 \cdot 10^{8} \mathrm{~cm}$ in einer Entfernung $d=90$ pc. Betrachtet man nun den Fluss des Systems im High State ebenfalls zu minimaler Phase, so ist dieser ungefähr doppelt so groß wie der Low State-Fluss. Wenn man berücksichtigt, dass bei der Systemgeometrie von AM Her die bestrahlte Fläche zur minimalen Phase sehr gering ist, stellt sich die Frage, woher der restliche Fluss entspringt.

1. Die Bestrahlung ist tatsächlich so hoch, dass selbst bei minimaler Phase das Doppelte des unbestrahlten Flusses erreicht wird.

2. Der Akkretionsstrom liefert einen signifikanten Beitrag in diesem Bereich.

Die Möglichkeit 1 kann verworfen werden. Möglichkeit 2 ist zumindest eine plausible Annahme für den fehlenden Flussanteil. In Gänsicke et al. (1995) wurde der Akkretionsstrom mit einem Schwarzkörper von $T=10000 \mathrm{~K}$ modelliert. Demnach 


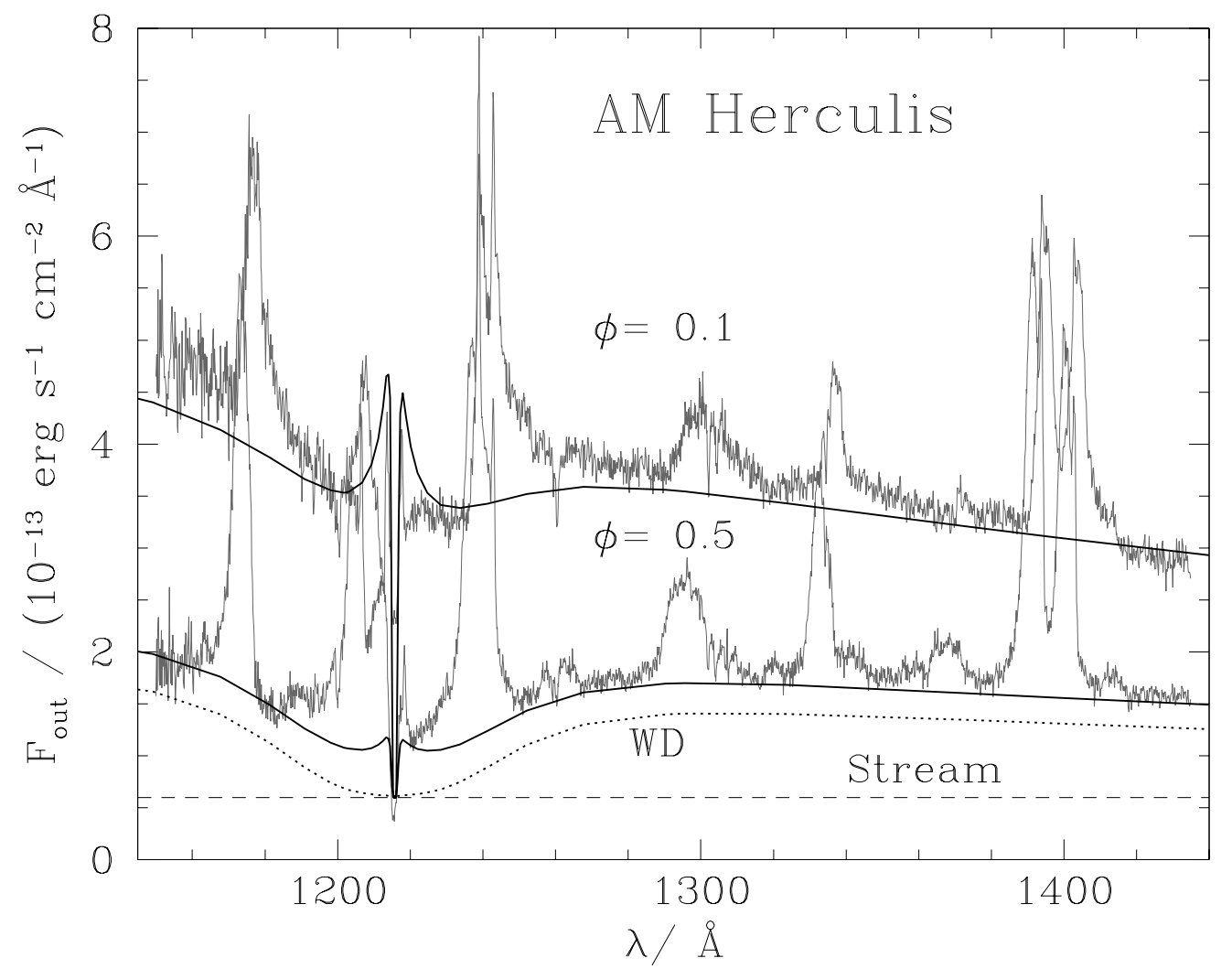

Abbildung 4.3: Vergleich der Modellspektren mit den HST/GHRS-Spektren zu den Phasen $\varphi_{\text {orb }}=0.1$ und $\varphi_{\text {orb }}=0.5$. Die Parameter des Modells sind $r_{\mathrm{wd}}=8.1 \cdot 10^{8} \mathrm{~cm}$, $d=90 \mathrm{pc}, i=40^{\circ}, \theta_{\mathrm{er}}=50^{\circ}, r_{\mathrm{er}}=0.3 r_{\mathrm{wd}}$ und $L=10^{33} \mathrm{erg} \mathrm{s}^{-1}$. Zu den Modellspektren wurde ein konstanter Stromanteil $F_{\text {stream }}=0.6 \cdot 10^{-13}$ hinzuaddiert (gestrichelte Linie). Die gepunktete Kurve repräsentiert zum Vergleich das Spektrum des unbestrahlten Weißen Zwerges mit einer Effektivtemperatur von $T_{\text {eff }}=20000 \mathrm{~K}$.

wäre der Stromanteil bei Wellenlängen $\lambda \approx 2900 \AA$ am größten und bei Wellenlängen $\lambda \lesssim 1500 \AA$ vernachlässigbar. Es ist jedoch nicht sicher, ob diese Annahme den Beitrag in dem hier betrachteten Wellenlängenbereich $1150 \AA \leq \lambda \leq 1450 \AA$ korrekt beschreibt. Der Anteil des Stroms in diesem Bereich könnte auch größer sein. Um den Strombeitrag zu berücksichtigen, wird dem Modellspektrum ein angenommener konstanter Flussbeitrag von $F_{\text {stream }}=0.6 \cdot 10^{-13} \mathrm{erg} \mathrm{s}^{-1} \mathrm{~cm}^{-2} \AA^{-1}$ hinzuaddiert. Dabei wird davon ausgegangen, dass der Strombeitrag unabhängig von der Phase und der Wellenlänge ist.

Die Geometrie des Modells zu verschiedenen Phasen findet sich in Abbildung 4.5. Hier sind das Gitter, die Emissionsregion und die Temperaturen der Flächenelemente in einem Bild zu sehen. Die Spektren im Vergleich mit den Beobachtungsdaten sind in Abbildung 4.3 dargestellt. Es wurden dabei die Phasen 


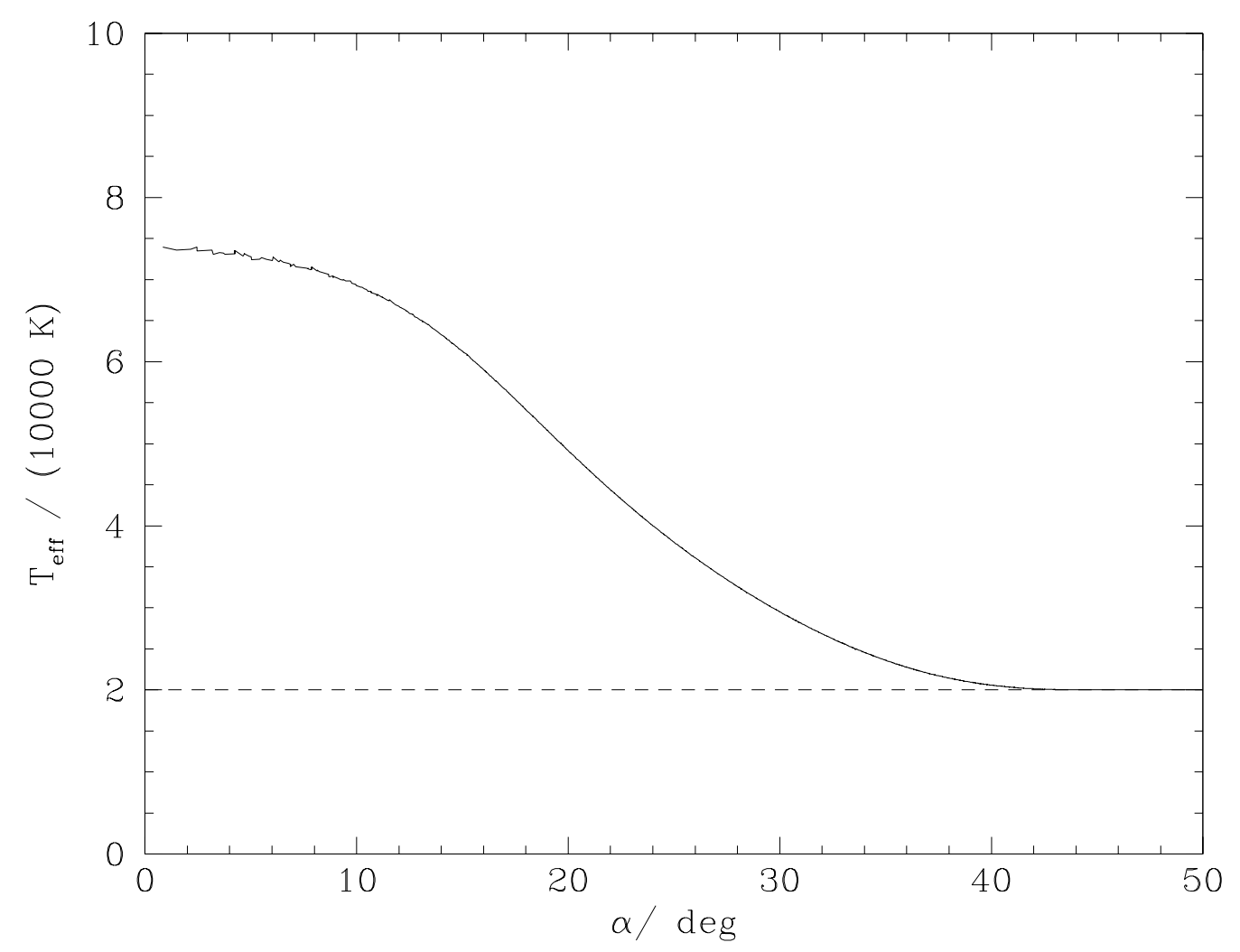

Abbildung 4.4: Temperaturen der einzelnen Flächenelemente für das Modell von AM Her.

$\varphi_{\text {orb }, 1}=0.1$ und $\varphi_{\text {orb }, 2}=0.5$ gewählt. Der Radius des Weißen Zwerges beträgt $r_{\mathrm{wd}}=8.1 \cdot 10^{8} \mathrm{~cm}$ bei einer Entfernung $d=90 \mathrm{pc}$ vom Beobachter. Die Inklination wurde etwas reduziert, um den sichtbaren bestrahlten Flächenanteil bei Phase $\varphi_{\text {orb }}=0.5 \mathrm{zu}$ erhöhen. Mit $i=40^{\circ}$ ist die Zwangsbedingung $i+\beta \gtrsim 90^{\circ}$ immer noch erfüllt bei einer Komplementbreite der Emissionsregion von $\theta_{\mathrm{er}}=50^{\circ}$. Der Radius der Emissionsregion beträgt nach wie vor $r_{\mathrm{er}}=0.3 r_{\mathrm{wd}}$. Die Leuchtkraft ist $L=1 \cdot 10^{33} \mathrm{erg} \mathrm{s}^{-1}$. Die grauen Kurven zeigen die Beobachtungsspektren zu den verschiedenen Phasen. Die schwarzen Kurven entsprechen den Modellspektren. Abbildung 4.4 zeigt zusätzlich noch die Verteilung der Effektivtemperatur in Abhängigkeit vom Winkel $\alpha$. 

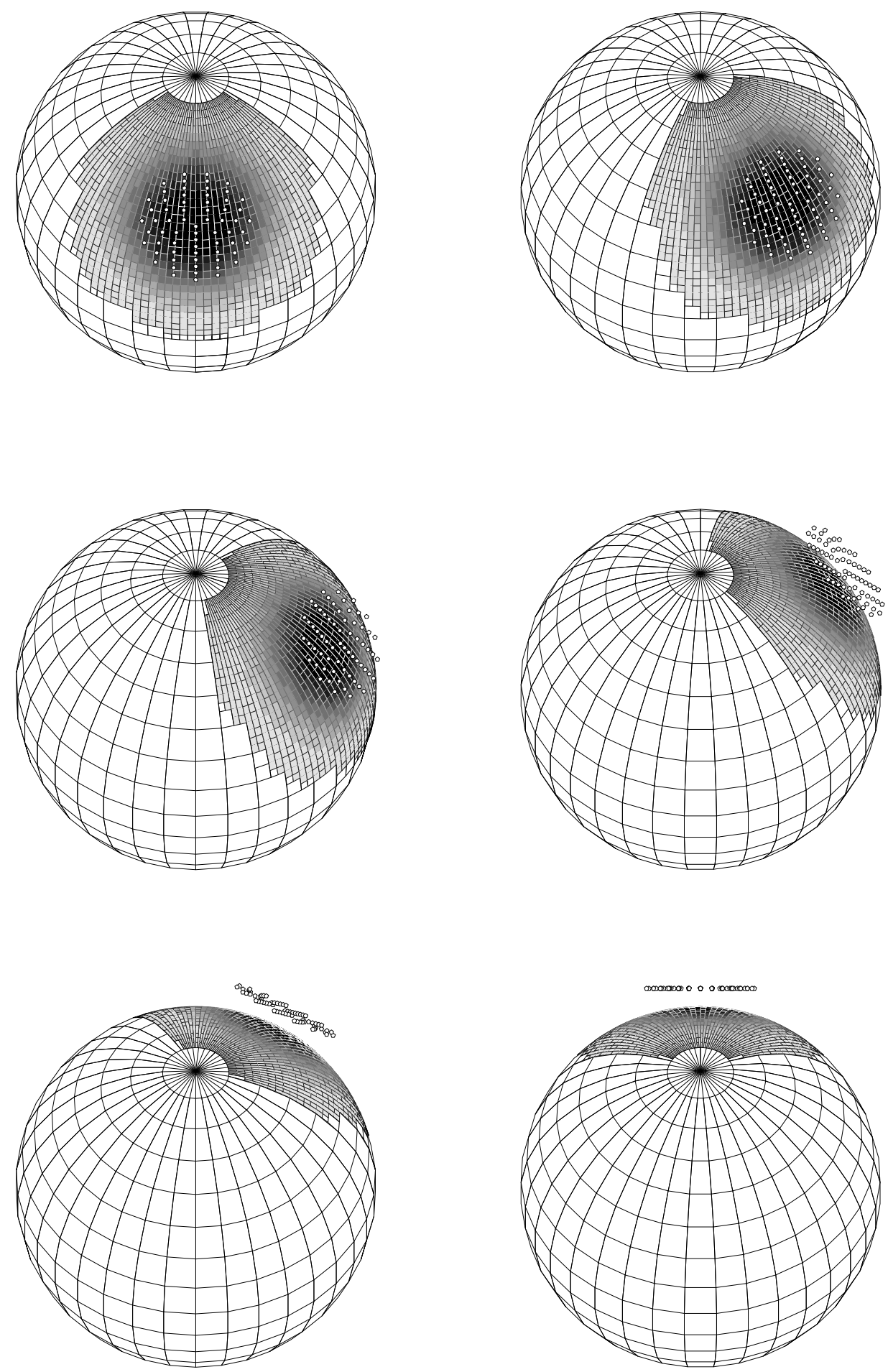

20000 74000

Abbildung 4.5: Gitter des Modells mit Emissionsregion (Punkte) zu verschiedenen Phasen. Die Temperaturen der bestrahlte Fläche sind als Graustufen kodiert. Die Werte der Temperaturen lassen sich Abbildung 4.4 entnehmen. Abgebildet sind von links oben über rechts oben nach rechts unten die Orbitalphasen $\varphi_{\text {orb }}=0.0 ; 0.1 ; 0.2 ; 0.3 ; 0.4 ; 0.5$. 


\subsection{Diskussion der Ergebnisse}

\subsubsection{Das Atmosphärenmodellgitter}

Der verwendete Atmosphärencode atX berechnet Atmosphären, die ausschließlich aus Wasserstoff bestehen. Ob die Anwendung dieser Modellatmosphären auf den Fall AM Her legitim ist, ist grundsätzlich nicht klar, da der Weiße Zwerg Materie vom Sekundärstern des Spektraltyps dM akkretiert. Allerdings sind beobachtete Eigenschaften der Atmosphäre, die durch schwere Elemente hervorgerufen werden könnten, sehr schwach und konzentrieren sich auf die Akkretionsregion. Die große nichtakkretierende bestrahlte Fläche hat weiterhin die Eigenschaften eines Weißen Zwerges des Spektraltyps DA (Gänsicke et al., 1995). In diesem dominieren die Wasserstofflinien (Koester \& Chanmugam, 1990).

Die Abhängigkeit der physikalischen Parameter ist in den Modellatmosphären reduziert auf eine Dimension. Da die geometrische Breite der Atmosphäre nur einen geringen Bruchteil $\left(\approx 10^{-4}-10^{-3}\right)$ des Radius des Weißen Zwerges ausmacht, ist dieser Ansatz gerechtfertigt.

Streuung der Strahlung durch Materie wird durch isotrope und kohärente Thomson-Streuung behandelt. Da diese Form der Streuung frequenzunabhängig ist und die Wellenlängen der gestreuten Photonen nicht verändert, lässt sich diese besonders effizient implementieren. Die Thomson-Streuung ist gültig für kleine Frequenzen $h \nu \ll m_{e} c^{2}$. Bei höheren Frequenzen gewinnt der Compton-Effekt an Relevanz, so dass zur Behandlung von Strahlung im Röntgenbereich Compton-Streuung berücksichtigt werden müsste. Da sich bei der Compton-Streuung die Frequenz der gestreuten Photonen abhängig vom Streuwinkel ändert, kann hier nicht mehr das besonders effiziente Rybicki-Verfahren zur Lösung der Strahlungstransportgleichung benutzt werden.

Weiterhin ist zu bemerken, dass die Modellatmosphären auf einen magnetischen Weißen Zwerg angewendet werden. In Atmosphären, die von einem Magnetfeld $B$ durchdrungen werden, liegt eine Zeeman-Aufspaltung der Energieniveaus vor. Im Allgemeinen erwartet man sichtbare Effekte bei Magnetfeldern $B \gtrsim 10^{6} \mathrm{G}$. Die Signifikanz der Effekte ist frequenzabhängig. Im UV-Spektralbereich sind sie für die Lyman-Linien bei einem Magnetfeld von $B \approx 10^{7} \mathrm{G}$ vernachlässigbar (Wickramasinghe \& Ferrario, 2000).

Als Grundlage für das Gitter wurde das Atmosphärenmodell eines Weißen Zwerges mit einer Effektivtemperatur von $T_{\text {eff }}=20000 \mathrm{~K}$ und einer Oberflächenschwerebeschleunigung von $\log \left[\mathrm{g} /\left(\mathrm{cm} \mathrm{s}^{-2}\right)\right]=8$ benutzt. Diese Werte beruhen auf Gänsicke et al. (1995) und stammen aus Beobachtungen im Zustand niedriger Akkretionsrate (Low State) des Systems. Dabei zeigte sich, dass diese Atmosphärenmodelle kompatibel mit den Beobachtungen sind. In Gänsicke et al. (1998) wurde bei der Modellierung des geheizten Flecks ein Wert von $T_{\text {eff }}=21000 \mathrm{~K}$ benutzt. 


\subsubsection{Die Einstrahlung}

Zyklotronstrahlung Mit dem Modell wurde die Bestrahlung der Atmosphäre mit der in der Akkretionsregion entstehenden Zyklotronstrahlung simuliert. Das Magnetfeld von AM Her wurde in Bailey et al. (1991) bestimmt mit $B=14.5 \mathrm{MG}$. Damit liegt die Fundamentalfrequenz der Zyklotronharmonischen bei $\lambda_{0}=73860 \AA$. Der Großteil der Strahlung wird jedoch in den Harmonischen abgestrahlt, so dass sich die Zyklotronstrahlung vom Infraroten bis zum optischen Bereich erstreckt. Die Approximation von Zyklotronstrahlung durch einen Schwarzkörper wurde in Kapitel 1.2 beschrieben. Das Spektrum der Zyklotronstrahlung wird im Modell mit einem Schwarzkörper der Temperatur $T_{\mathrm{bb}}=10000 \mathrm{~K}$ approximiert, der bei $\Lambda \approx 10^{7}$, einer Temperatur von $T \approx 20 \mathrm{keV}$ und dem Magnetfeld von $B=14.5 \mathrm{MG}$ gemäß den Approximationsformeln (1.13), (1.14) und (1.15) diesen Bereich beschreibt.

Das Zyklotronspektrum ist stark winkelabhängig in den Anteilen der Harmonischen, was bei dieser Approximation nicht berücksichtigt wird. Die Zyklotronleuchtkraft $L_{\text {cyc }}$ wurde auf der Basis von Beobachtungsdaten in Kapitel 1.2 mit $L_{\text {cyc }} \approx(2-3) \cdot 10^{32} \mathrm{erg} \mathrm{s}^{-1}$ abgeschätzt. In den Modellen zeigte sich, dass die benötigte Zyklotronleuchtkraft mit $L \approx 1 \cdot 10^{33} \mathrm{erg} \mathrm{s}^{-1}$ höher liegt, als sich durch die Beobachtungsabschätzungen rechtfertigen ließe, damit das Modellspektrum angepasst werden kann. Die Leuchtkraft der Zyklotronstrahlung ist allerdings nur ein Bruchteil der gesamten Akkretionsleuchtkraft. Daneben entsteht noch Bremsstrahlung, die im Modell nicht behandelt wird. Die Richtungsabhängigkeit der Zyklotronstrahlung führt dazu, dass ein Großteil der Strahlung senkrecht zum Magnetfeld abgestrahlt wird. Bei Masters et al. (1977) wird für die Abstrahlung eine Halbwertsbreite von $\delta \approx 90^{\circ}\left(\omega / \omega_{c}\right)^{-1 / 6}(k T /(64 \mathrm{keV}))^{1 / 3}$ angegeben. Bei Annahme einer isotropen Abstrahlung wird die Gesamtleuchtkraft der Zyklotronstrahlung überschätzt.

Bremsstrahlung Wie schon erwähnt, entsteht in der Akkretionsregion im Bereich von $2-60 \mathrm{keV}$ Röntgenstrahlung, die einer Bremsstrahlungstemperatur von $T_{\mathrm{X}} \approx 10^{9} \mathrm{~K}$ entspricht (Chanmugam \& Wagner, 1978). Diese bestrahlt zusätzlich zur Zyklontronstrahlung die Oberfläche des Weißen Zwerges. Allerdings ist der Teil der Emissionsregion, der Bremsstrahlung emittiert, wesenlich kleiner und liegt typischerweise bei $f=A_{\mathrm{bs}} / A_{\mathrm{wd}} \approx 10^{-5}$ (Beuermann, 1988). Die Region liegt konzentriert im Kern der Akkretionssäule bei hohen Massenflussraten $\dot{m}$. Bei Atmosphären aus reinem Wasserstoff nimmt die Opazität im Wellenlängenbereich unterhalb der Lymankante ab, so dass die Strahlung in diesem Bereich kaum in der Atmosphäre absorbiert wird. Da im Zentrum des Akkretionsbereichs die Häufigkeit von schwereren Elementen angenommen werden muss, reicht eine Behandlung durch eine reine Wasserstoffatmosphäre nicht aus. Eventuell spielen auch noch andere Effekte wie der ComptonEffekt durch Streuung an freien Elektronen eine Rolle (van Teeseling et al., 1994). Effekte in der Atmosphärenstruktur, die durch die Bremsstrahlung hervorgerufen werden, fehlen in unserem Modell. 


\subsubsection{Akkretionsregion}

Die Emissionsregion des Modells besteht aus isotrop strahlenden Punktquellen mit homogener Leuchtkraftverteilung. Es stellt sich die Frage, inwieweit ein solches Modell ein legitimes Abbild des strahlungserzeugenden Bereichs der Akkretionsregion darstellt. Aufgrund verschiedener Beobachtungsbefunde muss man davon ausgehen, dass die Akkretionsregion eine komplexere Struktur besitzt, so dass die Annahme einer über die Akkretionsfläche konstanten Akkretionsrate nur eine Näherung ist. Die hier betrachtete Zyklotronstrahlung dominiert bei geringeren Massenstromdichten $\dot{m} \lesssim 0.1 \mathrm{~g} \mathrm{~cm}^{-2} \mathrm{~s}^{-1}$ (bei einem Magnetfeld von $B=10 \mathrm{MG}$ ), wohingegen die Bremstrahlung bei höheren $\dot{m}$ dominiert (Woelk \& Beuermann, 1996). Weiterhin findet man Fluktuationen der Leuchtkraft auf sehr kurzen Zeitskalen im Bereich von Minuten, so dass die Annahme einer stationären Akkretion an einem Ort nicht gerechtfertigt erscheint.

Die Modellierung bezieht sich auf eine mittlere Akkretionsrate die durch eine zeitlichen Mittelung auf der Zeitskala der Fluktuationen und einer örtlichen Mittelung über die Akkretionsfläche entsteht. In diesem Sinne ist die Emissionsregion des Modells nicht mit einer isomorphen Abbildung der Akkretionsregion zu verwechseln.

\subsubsection{Systemgeometrie}

In Gänsicke et al. (2001) finden sich eine Reihe von Angaben zur Systemgeometrie von AM Her mit einerseits der Inklination $i=35^{\circ}$ und der Komplementbreite der Akkretionssäule $\beta=58^{\circ}$ und andererseits $i \approx 50^{\circ}$ und $\beta \approx 50-60^{\circ}$. Die Zwangsbedingung $i+\beta \gtrsim 90^{\circ}$ folgt aus der beobachteten Bedeckung der Röntgenstrahlungsemissionsregion und der EUV-Emissionsregion (Paerels et al., 1996). Es ist jedoch auch zu berücksichtigen, dass teilweise die einzelnen Emissionsregionen nicht vollständig bedeckt sind (Bailey et al., 1984) und die Zyklotronstrahlung bei $\varphi_{\text {orb }}=0.5$ zwar ein lokales Minimum besitzt, jedoch deutlich sichtbar ist. Weiterhin muss $i<\beta$ gelten, da ansonsten eine Bedeckung der Emissionsregion durch den Akkretionsstrom erfolgen müsste, die man nicht beobachtet. Im Modell wird die Komplementbreite $\beta$ mit dem Winkel $\theta_{\text {er }}$ identifiziert. Die verwendeteten Parameter des Modells für AM Her mit der Inklination $i=40^{\circ}$ und $\theta_{\mathrm{er}}=50^{\circ}$ sind konsistent mit den Zwangsbedingungen. 


\section{Kapitel 5}

\section{Zusammenfassung und Ausblick}

\subsection{Zusammenfassung}

Polare bzw. AM Her Systeme sind halbgetrennte enge Doppelsternsysteme bestehend aus einem Weißen Zwerg und einem späten Hauptreihenstern als Sekundärstern. Der Sekundärstern füllt sein gesamtes Roche-Volumen aus, so dass Materie transferiert wird, die der Weiße Zwerg akkretiert. Dieser besitzt ein starkes Magnetfeld, so dass die Ausbildung einer Akkretionsscheibe vollständig verhindert wird. Der Materiestrom fließt entlang der Magnetfeldlinien und trifft lokalisiert in der Umgebung des/der magnetischen Pols/Pole auf die Oberfläche des Weißen Zwergs. In einer Stoßwelle über der Oberfläche wird der Materiestrom abgebremst und erhitzt. In der Stoßregion entstehen Bremsstrahlung im harten Röntgenbereich und polarisierte Zyklotronstrahlung im optischen bis infraroten Spektralbereich, die die Atmosphäre des Weißen Zwerges bestrahlen. Es gibt phasenaufgelöste Spektren von AM Her im ultravioletten Spektralbereich, in dem die Atmosphäre des Weißen Zwerges den Strahlungsfluss dominiert, die eine sinusförmige Modulation zeigen. Diese ist auf eine Heizung der Atmosphäre zurückzuführen. Weiterhin zeigen diese Beobachtungen, dass die für einen Weißen Zwerg aufgrund der starken Oberflächenschwerebeschleunigung sehr breite Ly $\alpha$-Absorptionslinie aufgefüllt ist. Es besteht die Vermutung, dass diese Effekte durch die Erhitzung der Atmosphäre durch die in der Akkretionsregion enstehenden Strahlung verursacht wird. In dieser Arbeit wurde die Wirkung der Zyklotronstrahlung auf die Atmosphäre des Weißen Zwerges betrachtet.

Zur Berechnung der Atmosphäre eines Weißen Zwergs wurde ein bestehender eindimensionaler LTE Code verwendet. Dieser Code wurde modifiziert, um bestrahlte Atmosphären behandeln zu können. Ausgehend von Modellen mit isotroper Einstrahlung wurde die Reaktion der Atmosphären untersucht. Die Modelle zeigten für moderate Bestrahlungsflüsse eine konstante Temperaturzunahme bis zu einer Grenztiefe, in der die Temperaturschichtung in die der ungestörten Atmosphäre mündet. Bei höheren Bestrahlungsflüssen entwickelt die Schichtung eine Temperaturinversion. Dazu zeigt sich im Spektrum der Atmosphäre eine Modifikation der Absorpti- 
onslinien. Die Linien werden allmählich aufgefüllt und entwickeln sich parallel zur Herausbildung der Temperaturinversion zu Emissionslinien. Bei winkelabhängiger Bestrahlung zeigen sich zusätzlich noch richtungsabhängige Effekte. Bestrahlung unter flachen Winkeln tendiert schneller zu einer Ausbildung von Emission im Linienkern. Senkrechte Bestrahlung dringt tiefer in die Atmosphäre ein und führt eher zu einer Auffüllung der Linienflügel.

Mit den Parametern des Weißen Zwerges in AM Her wurde ein winkelabhängiges Atmosphärenmodellgitter mit verschiedenen Bestrahlungsflüssen gerechnet. Auf Basis dieses Gitters wurde ein Modell erstellt, welches einen Modellstern mit einer externen Emissionsregion simuliert, um ein Gesamtspektrum zu erhalten, welches mit den phasenaufgelösten Beobachtungsdaten von AM Her verglichen wurde. Es ist möglich, die beobachteten Spektren mit einem Modell zu erklären, das in den bekannten Parametern in der gleichen Größenordnung liegt. Allerdings muss zu einer konsistenten Modellierung der AM Her-Daten noch ein Flussbeitrag durch den Akkretionsstrom angenommen werden.

\subsection{Ausblick}

Obwohl die Beobachtungen von AM Her hinreichend mit dem Modell erklärt werden können, handelt es sich noch um eine grobe Näherung der Prozesse, die in diesem System vorliegen. Das Modell unterliegt einer Vielzahl von Approximationen, die aus Gründen der Effizienz angenommen werden. Andere Vereinfachungen wiederum werden eingeführt, um die Aussagekraft des Modells nicht überzubelasten.

Das Gitter der bestrahlten Modellatmosphären wurde mit einem modifizierten LTE-Code berechnet. Obwohl NLTE-Effekte bei unbestrahlten Weißen Zwergen in diesem Temperaturbereich vernachlässigbar sind, ist nicht klar, ob NLTE-Effekte bei starker Bestrahlung auftreten, die zu einer sehr heißen Schicht bei optisch dünnen Tiefen führt. Hier wären noch Studien von bestrahlten Modellatmosphären von Interesse, die mit einem NLTE-Code berechnet werden.

Die ausgedehnte Emissionsregion wurde als Menge von isotrop strahlenden Punktquellen mit homogener Leuchtkraftverteilung modelliert. Es wurde ein Modellatmosphärengitter benutzt, bei dem Bestrahlung immer nur unter einem bestimmten Winkel erfolgt. Dabei wurde im Modell ein flussgewichteter gemittelter Bestrahlungswinkel benutzt, um die Bestrahlung über einen Winkelbereich auf einen Winkel zu reduzieren. Hier könnte man versuchen eine Einstrahlung mit einer Winkelverteilung zu verwenden, die die Verteilung der Punktquellen besser abbildet. Solche Modellatmosphären müssten allerdings bedarfsweise gerechnet werden, was den Zeitaufwand zur Berechnung eines Modells stark vergrößert.

Weiterhin wird vernachlässigt, dass die Zyklotronstrahlung gerichtet erfolgt mit einer in der Frequenz variierenden Winkelabhängigkeit. Denkbar wäre hier eine Verknüpfung mit der Modellierung einer ausgedehnten Akkretionssäule, die winkelabhängige Strahlung emittiert. Eine vollständige Modellierung der Strahlungs- 
prozesse in der Akkretionsregion müsste zusätzlich noch die entstehende Bremstrahlung in Betracht ziehen, die allerdings bei Atmosphären, die aus reinem Wasserstoff bestehen, aufgrund der zu geringen Opazität in diesem Bereich zu Problemen führt.

Von grossem Interesse wäre die Betrachtung weiterer hochaufgelöster phasenabhängiger spektroskopischer Daten im UV-Bereich von kataklysmischen Veränderlichen, um die Erhitzung der Weißen Zwerg Atmosphären genauer zu verifizieren. Um UV-Daten zu erhalten, ist man auf Satelliten angewiesen, da für diesen Bereich die Erdatmosphäre undurchsichtig ist. Der Space Telescope Imaging spectrograph (STIS), der den Goddard high-resolution spectrograph (GHRS) auf dem HubbleSpace Telescope 1997 ersetzt hat, hat im August 2004 aufgrund eines Defekts seine Arbeit eingestellt. Aus diesem Grund ist mit neuen Daten in nächster Zeit nicht zu rechen. Neuere, leider nicht phasenaufgelöste, spektroskopische Beobachtungen von kataklysmischen Veränderlichen, die mit STIS aufgenommen wurden, untermauern das an AM Her untersuchte Phänomen der Auffüllung der Ly $\alpha$-Linie im Zustand hoher Akkretionsrate (Araujo-Betancor et al., im Druck) anhand der drei im High State beobachteten Polare CD Ind, UW Pic und AN UMa. Teilweise ist hier im Fall CD Ind sogar breite Emission in der Linie zu erkennen, deren mögliche Herkunft aus der geheizten Atmosphäre untersucht werden müsste. 


\section{Anhang A}

\section{wd_model Referenz}

\section{A.1 Organisation der Daten}

Die eindimensionalen Modellatmosphären werden in einer hierarchischen Verzeichnisstruktur organisisiert. Die Struktur soll im folgenden beschrieben werden. Das oberste Verzeichnis der Struktur sei BASEPATH. Innerhalb von BASEPATH folgt eine Reihe von $N_{\mu}$ Unterverzeichnissen, die jeweils einen bestimmten Bestrahlungswinkel zugeordnet sind. Die Namensgebung ist definiert durch die Syntax aI, wobei $I$ den Winkelindex darstellt. Innerhalb der Winkelverzeichnisse gibt es wiederum eine Anzahl von Unterverzeichnissen, deren Namen dadurch gegeben sind, dass sie ausschließlich aus Ziffern besteht. Ein solches Verzeichnis enthält jeweils die atX Ausgabefiles für einen bestimmten Bestrahlungsfluss. Der aus Ziffern bestehende Name eines solchen Verzeichnis dient lediglich der Differenzierung. Die Daten sind demnach in einem 2-dimensionalen Gitter organisiert, das jeweils für einen Bestrahlungswinkel $\mu_{i}$ eine bestimmte Anzahl von Modellatmosphären enthält, die jeweils einem bestimmten Bestrahlungsfluss $F_{\text {in }}$ zugehörig sind. Die schematische Struktur der Verzeichnisebenen ist in Abbildung A.1 dargestellt.

\section{A.2 wd_model.in Format}

Beim Start von wd_model wird das Parameterfile wd_model.in eingelesen, welches sich im aktuellen Arbeitsverzeichnis befinden muss. In diesem File sind alle Parameter enthalten, die zur Spezifikation eines Modells notwendig sind.

\section{Format}

Die Definition eines Parameters <parameter-name> mit dem Wert <parameter-value> erfolgt in einer Zeile

$\langle$ line $>=*$ WSP $[$ <assignment $>$ [ <comment> ] CRLF 


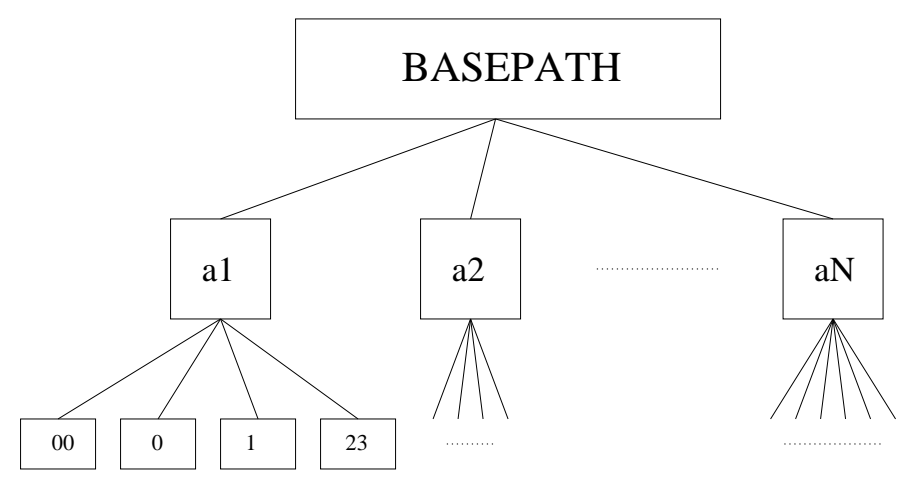

Abbildung A.1: Hierarchie der Verzeichnisstruktur der Modellatmosphärendatenbank.

durch die Regel ${ }^{1}$

<assignment $>=\langle$ parameter-name $>$ *WSP $"=" *$ WSP $<$ parameter-value $>$

Ein legaler Parametername wird gebildet durch

$\langle$ parameter-name $>=$ ALPHA $*($ ALPHA / DIGIT / "_" / "-" )

Ein legaler Wert ist durch

<parameter-value $>=1 *($ ALPHA / DIGIT / "." / "_" / "/ / "-" / DQUOTE / SP )

gegeben. Kommentare werden durch ein '\#' eingeleitet und reichen bis zum Ende der Zeile.

$<$ comment $>=" \# " *($ VCHAR / WSP $)$

\section{A.3 wd_model Parameter}

\section{Allgemeine Parameter}

basepath $=$ String

Dieser Parameter definiert das Basis Verzeichnis, in dem sich die Modellatmosphären befinden. In diesem Verzeichnis befindet sich eine Reihe von Unterverzeichnissen, die jeweils einem Bestrahlungswinkel zugeordnet sind.

model_file = String

Basisname des atX Modellfiles (z.B. 't20000g80')

\footnotetext{
${ }^{1}$ Wir benutzen hier die „Augmented Backus-Naur Form“ (ABNF), wie sie in Crocker \& Overell spezifiziert ist.
} 


\section{Numerische Parameter}

number_of_angles $=$ Integer Number

Spezifiziert die Anzahl der in at verwendeten Winkel.

mun $=$ Real Number

Hierbei handelt es sich um number_of_angles Parameterdefinitionen. $n$ nimmt den Wert von 1 bis number_of_angles an und bezeichnet den Winkelindex. Es wird jeweils der Kosinus $\mu_{n}$ des Winkels $\theta_{n}$ angegeben.

$\mathrm{w} n=$ Real Number

Entsprechend zu den Winkelkosinus Definitionen mun werden hier die jeweiligen Integrationsgewichte angegeben.

ntheta $=$ Integer Number

Anzahl der Diskretisierungspunkte der Kugelkoordinate $\theta$ für das Modell

nphi = Integer Number

Anzahl der Diskretisierungspunkte der Kugelkoordinate $\varphi$ für das Modell

spot_refinements = Integer Number

Die Gitterstruktur wird in der Umgebung der bestrahlten Region einer Verfeinerung unterzogen. Ein Verfeinerungsdurchlauf entspricht einer Teilung der Flächenelemente, für die eine Punktquelle sichtbar ist, in vier Teile. Der Parameter definiert die Anzahl der Verfeinerungsiterationen.

\section{Physikalische Parameter}

wd_radius $=$ Real Number $/ \mathrm{cm}$

Definiert den Radius des Sterns.

wd_temperature $=$ Real Number $/ \mathrm{K}$

Effektivtemperatur des Weißen Zwerges.

spot_separation_temperature $=$ Real Number $/ \mathrm{K}$

Es werden zwei Ausgabespektren erstellt. Eins enthält den Anteil der unbestrahlten Oberfläche des Weißen Zwerges; das andere beschreibt den geheizten Fleck. Durch diesen Parameter wird die Grenztemperatur definiert, bei deren Überschreiten das Spektrum des Flächenelementes zum Fleck gerechnet wird.

phase_start

phase_end

phase_step $=$ Real Number

Definiert Anfang und Ende der Phasenschleife, die mit der ensprechenden Schrittweite durchlaufen wird.

distance $=$ Real Number / parsec

Entfernung des Systems.

inclination $=$ Real Number $/$ degree 
Inklination des Systems, d.h. Winkel zwischen Beobachterrichtung und Normalen der Orbitalebene des Systems.

\section{Beschreibung der Bestrahlungsquelle}

source_type = point $\mid$ circular_area $\mid$ circle

Beschreibt die geometrische Form der Emissionsregion. Es werden zur exakten Spezifikation der Emissionsregion weitere Parameter benötigt, die abhängig sind vom Typ der Region.

source_theta $=$ Real Number $/$ degree

Entspricht der Kugelkoordinate $\theta$ des Mittelpunktes der Emissionsregion.

source_phi $=$ Real Number / degree

Enstspricht der Kugelkoordinate $\varphi$ des Mittelpunktes der Emissionsregion.

source_height = Real Number / wd_radius

Bestimmt die Höhe der Emissionsregion über der Oberfläche in der Einheit Sternradius.

point

Hier werden keine weiteren Parameter benötigt.

circle

source_circle_radius $=$ Real Number $/$ wd_radius

Radius der Kreisfläche in der Einheit Sternradius.

source_npoints = Integer Number

Anzahl der Punktquellen.

circular_area

source_circle_radius = Real Number / wd_radius

Radius der Kreisfläche in der Einheit Sternradius.

source_ncircles $=$ Integer Number

Anzahl der Kreisringe.

source_npoints = Integer Number

Anzahl der Punktquellen.

Weiterhin muss die Leuchtkraft der Emissionregion definiert werden. Dies geschieht durch folgende Parameter:

source_luminosity = Real Number $/(\mathrm{erg} / \mathrm{s})$

Gibt die Luminosität an.

source_luminosity_distribution = homogenous

Definiert die Art der Verteilung. Eine Homogene Verteilung, die allen Punktquellen dieselbe Leuchtkraft zuordnet, ist zur Zeit die einzige Möglichkeit. 


\section{Ausgabeparameter}

spec_angstrom_flux

Wellenlänge $\lambda$ in $\AA$, Fluß $F$ in $\operatorname{erg~s}^{-1} \mathrm{~cm}^{-2} \AA$

spec_angstrom_apflux

Wellenlänge $\lambda$ in $\AA$, astrophysikalischer Fluß $F_{\text {ap }}$ in $\operatorname{erg~s}^{-1} \mathrm{~cm}^{-2} \AA$

spec_hz_flux

Frequenz $\nu$ in $\mathrm{Hz}$, Fluß $F$ in $\operatorname{erg~s}^{-1} \mathrm{~cm}^{-2} \AA$

spec_hz_apflux $=$ yes $\mid$ no

Frequenz $\nu$ in $\mathrm{Hz}$, astrophysikalischer Fluß $F_{\text {ap }}$ in $\operatorname{erg~s}^{-1} \mathrm{~cm}^{-2} \mathrm{~Hz}$

Jeder dieser Parameter kann auf yes oder no gesetzt werden, um das entsprechende Ausgabefile im angegebenen Format zu erhalten.

use_distance_scaling $=$ yes $\mid$ no

Skaliert die Ausgabespektren auf die angegebene Systemdistanz.

\section{A.4 wd_model Ausgabe}

Die Berechnungen von wd_model werden in verschiedenen Ausgabefiles festgehalten, die im Folgenden beschrieben werden.

\section{A.4.1 Informationen über Flächenelemente}

File: sphere-theo.dat

Dieses File enthält verschiedene Daten über die Flächenelemente, die direkt berechnet werden können. Format:

\begin{tabular}{c|c|c|c}
$\alpha$ & $\theta_{\text {in }}$ & $d$ & $F_{\text {ap,in }}$ \\
\hline $\operatorname{deg}$ & $\operatorname{deg}$ & $r_{\text {wd }}$ & $\operatorname{erg~s}^{-1} \mathrm{~cm}^{-2}$
\end{tabular}

Der Winkel $\alpha$ entspricht dem Winkel zwischen dem Flächenelementvektor und dem Mittelpunkt der Emissionsregion. $\theta_{\text {in }}$ enstspricht dem Bestrahlungswinkel. $d$ ist der Abstand zwischen Mittelpunkt der Emissionsregion und Position des Flächenelements in der Einheit des Radius. $F_{\text {in }}$ ist der Bestrahlungsfluss für das jeweilige Flächenelement.

File: sphere-calc.dat

Dieses File enthält Daten, die aus dem Datensatz entnommen sind, bzw. den diskretisierten Größen entsprechen. Format:

\begin{tabular}{c|c|c|c|c}
$\alpha$ & $\theta_{\text {in }}$ & $F_{\text {ap,in }}$ & $F_{\text {ap }, \text { out }}$ & $F_{\text {ap }}$ \\
\hline $\operatorname{deg}$ & $\operatorname{deg}$ & $\operatorname{erg~s}^{-1} \mathrm{~cm}^{-2}$ & $\operatorname{erg~s}^{-1} \mathrm{~cm}^{-2}$ & $\operatorname{erg~s}^{-1} \mathrm{~cm}^{-2}$
\end{tabular}


Der Winkel $\theta_{\text {in }}$ ist nun der diskretisierte Bestrahlungswinkel. $F_{\text {in }}$ enspricht dem Bestrahlungsfluss des ausgewählten Datensatzes, ebenso wie $F_{\text {out }}$ dem dazugehörigen ausgehenden Fluss entspricht. $F=F_{\text {in }}+F_{\text {out }}$ ist der Gesamtfluss, der durch die Effektivtemperatur $T_{\text {eff }}$ gegeben ist.

\section{A.4.2 Spektrale Daten}

File: flux.x_unit.y_unit.phase_index

Der genaue Wert des Filenamens hängt hier ab, von den im Parameterfile gesetzten Einheiten. Folgende Parameter können jeweils auf yes oder no gesetzt werden:

- spec_hz_flux

- spec_hz_apflux

- spec_angstrom_flux

- spec_angstrom_apflux

x_unit ist 'hz' oder 'a' und y_unit entweder 'f' oder 'apf'. Das Format des Files ist eine einfache Tabelle mit zwei Spalten. Die erste Spalte gibt entweder die Frequenz $\nu /$ Hz oder die Wellenlänge $\lambda / \AA$ an.

Das File enthält das Spektrum des gesamten Weißen Zwerges, das mit der flussbasierten Näherungsmethode berechnet wurde.

spectrum_total.x_unit.y_unit.phase_index

Dieses File enthält das Gesamtspektrum des Weißen Zwerges, berechnet auf Basis winkelabhängiger Intensitätsinformation.

spectrum_spot.x_unit.y_unit.phase_index

Enthält das Spektrum des heißen Flecks. Die Temperaturgrenze $T_{\text {spot }}$ wird durch den Parameter spot_separation_temperature definiert.

spectrum_wd.x_unit.y_unit.phase_index

Enthält das Spektrum der unbestrahlten Oberfläche des Weißen Zwerges. 


\section{Literaturverzeichnis}

Aizu, K., 1973. X-Ray Emission Region of a White Dwarf with Accretion. Prog. Theor. Phys. 49, 1184.

Araujo-Betancor, S., Gänsicke, B. T., Long, K. S., Beuermann, K., de Martino, D., Sion, E. S., Szkody, P., im Druck. Far-ultraviolet Spectroscopy of Magnetic Cataclysmic Variables. ApJ .

Bailey, J., Ferrario, L., Wickramasinghe, D. T., 1991. Cyclotron features in the infrared spectrum of AM Herculis. MNRAS 251, 37P.

Bailey, J., Hough, J. H., Gilmozzi, R., Axon, D. J., 1984. Infrared and optical polarimetry of AM Herculis. MNRAS 207, 777.

Beuermann, K., The X-ray properties of AM HER stars, S. 125 (Polarized Radiation of Circumstellar Origin 1988).

Beuermann, K., Radiation-hydrodynamic Models of the Accretion Spots in Magnetic Cataclysmic Variables. In S. Vrielmann, M. Cropper (Hg.), Magnetic Cataclysmic Variables, S. 187 (2004).

Bohlin, R. C., 1973. Mariner 9 ultraviolet spectrometer experiment : interstellar absorption at Lyman alpha in OB stars. ApJ 182, 139.

Chanmugam, G., Langer, S. H., 1991. Formulae for the cyclotron luminosity of magnetized plasma slabs. ApJ 368, 580.

Chanmugam, G., Wagner, R. L., 1978. The remarkable system AM Herculis/3U 1809+50. II - A single accretion column model. ApJ 222, 641.

Chanmugam, G., Wagner, R. L., 1979. Cyclotron self-absorption in accretion columns of magnetic degenerate stars. ApJ 232, 895.

Crocker, D., Overell, P., 1997. RFC2234, Augmented BNF for Syntax Specifications: $A B N F$.

Fabian, A. C., Pringle, J. E., Rees, M. J., 1976. X-ray emission from accretion on to white dwarfs. MNRAS 175, 43. 
Feautrier, P., 1964. Sur la résolution numérique de l'équation de transfert. C. R. Acad. Sc. Paris 258, 3189.

Fischer, A., 2000. Zur Strahlungshydrodynamik der Akkretionssäulen auf magnetischen Weißen Zwergen. Dissertation, Georg-August Universität Göttingen.

Frank, J., King, A., Raine, D., Accretion Power in Astrophysics (Cambridge University Press 1992), zweite Auflage.

Gänsicke, B. T., Fischer, A., Silvotti, R., de Martino, D., 2001. A model for the optical high state light curve of AM Herculis. A\&A 372, 557.

Gänsicke, B. T., 1993. Berechnung von Atmosphären weißer Zwerge mit H-line blanketing und Anwendung auf kataklysmische Veränderliche. Diplomarbeit, Institut für Astronomie und Astrophysik TU Berlin.

Gänsicke, B. T., Beuermann, K., de Martino, D., 1995. The white dwarf in AM Herculis. A\&A 303, 127.

Gänsicke, B. T., Hoard, D. W., Beuermann, K., Sion, E. M., Szkody, P., 1998. HST/GHRS observations of AM Herculis. A\&A 338, 933.

Gehren, T., 1975. LTE-Sternatmosphärenmodelle. Unveröffentlichtes Skript.

Greeley, B. W., Blair, W. P., Long, K. S., Raymond, J. C., 1999. The Far-Ultraviolet Spectrum and Short-Timescale Variability of AM Herculis from Observations with the Hopkins Ultraviolet Telescope. ApJ 513, 491.

Hessman, F. V., Gänsicke, B. T., Mattei, J. A., 2000. The history and source of mass-transfer variations in AM Herculis. A\&A 361, 952.

Hoshi, R., 1973. X-Ray Emission from White Dwarfs in Close Binary Systems. Prog. Theor. Phys. 49, 776.

Hubeny, I., Accelerated Lambda Iteration: An Overview. In ASP Conf. Ser. 288: Stellar Atmosphere Modeling, S. 17 (2003).

König, M., Gänsicke, B., Heating of a WD Atmosphere by Irradiation with Cyclotron Emission. In ASP Conf. Ser. 288: Stellar Atmosphere Modeling, S. 629 (2003).

King, A. R., Lasota, J. P., 1979. Accretion on to highly magnetized white dwarfs. MNRAS 188, 653.

Koester, D., Chanmugam, G., 1990. Physics of white dwarf stars. Reports of Progress in Physics 53, 837.

Lamb, D. Q., Masters, A. R., 1979. X and UV radiation from accreting magnetic degenerate dwarfs. ApJ 234, L117. 
Madej, J., Różańska, A., 2000. X-ray irradiated model stellar atmospheres. A\&A 356,654 .

Masters, A. R., Fabian, A. C., Rees, M. J., Pringle, J. E., 1977. Cyclotron emission from accreting magnetic white dwarfs. MNRAS 178, 501.

Mauche, C. W., Raymond, J. C., 1998. ORFEUS II Far-Ultraviolet Spectroscopy of AM Herculis. ApJ 505, 869.

Mihalas, D., Stellar atmospheres (San Francisco, W. H. Freeman and Co. 1978), zweite Auflage.

Napiwotzki, R., 1997. LTE or NLTE for the analysis of hot white dwarf and subdwarf $B$ stars? A\&A 322, 256.

Paerels, F., Hur, M. Y., Mauche, C. W., Heise, J., 1996. Extreme Ultraviolet Spectroscopy of the White Dwarf Photosphere in AM Herculis. ApJ 464, 884.

Ramsay, G., Mason, K. O., Cropper, M., Watson, M. G., Clayton, K. L., 1994. ROSAT Observations of An-Ursae and Mr-Serpentis - the Status of the Soft XRay Excess in AM Herculis Stars. MNRAS 270, 692.

Raymond, J. C., Mauche, C. W., Bowyer, S., Hurwitz, M., 1995. ORFEUS Observations of AM Herculis. ApJ 440, 331.

Ritter, H., Kolb, U., 1998. Catalogue of cataclysmic binaries, low-mass X-ray binaries and related objects (Sixth edition). A\&AS 129, 83.

Rybicki, G. B., 1971. A Modified Feautrier Method. J. Quant. Radiat. Transfer 11, 589.

van Teeseling, A., Heise, J., Paerels, F., 1994. X-ray irradiation of white dwarf atmospheres: The soft X-ray spectrum of AM Herculis. A\&A 281, 119.

Warner, B., Cataclysmic Variable Stars (Cambridge University Press 1995).

Wickramasinghe, D. T., Ferrario, L., 2000. Magnetism in Isolated and Binary White Dwarfs. PASP 112, 873.

Wickramasinghe, D. T., Meggitt, S. M. A., 1985. An analysis of the AM Her-type variables $C W 1103+254$ and $E$ 1405-451. MNRAS 216, 857.

Woelk, U., Beuermann, K., 1996. Stationary radiation hydrodynamics of accreting magnetic white dwarfs. A\&A 306, 232.

Wu, K., 2000. Accretion onto Magnetic White Dwarfs. Space Science Reviews 93, 611. 


\section{Danksagung}

Ich danke Prof. Dr. Klaus Beuermann und PD Dr. Boris Gänsicke für die Betreuung der Arbeit und die zahlreichen Hinweise.

Die Arbeit wurde gefördert vom Graduiertenkolleg für Strömungsinstabilitäten und Turbulenz an der Universität Göttingen.

Ganz herzlich danke ich meiner Familie für die vielfältige Unterstützung in jeder Hinsicht. Den Kollegen der Arbeitsgruppe sei gedankt für die zahlreichen Korrekturhinweise. Allen Freunden, die mich mit ihrer moralischen Unterstützung begleitet haben, gebührt mehr Dank als durch Worte ausdrückbar ist. 


\section{Lebenslauf}

Daten

Name

Matthias König

Geburtsdatum

15. Januar 1973

Geburtsort

Hofgeismar

Staatsangehörigkeit

Familienstand

Deutsch

ledig

\section{Schulausbildung}

1979-1983

1983-1989

1989-1992

\section{Studium}

1992-2000

1995

1999-2000

2000

2000-2003

Seit 2000
Grundschule Liebenau

Gustav-Heinemann-Schule, Hofgeismar

Albert-Schweitzer-Schule, Hofgeismar

Abschluss: Allgemeine Hochschulreife

Physikstudium an der Georg-August-Universität Göttingen Vordiplomsprüfung

Studentische Hilfskraft am Institut für Theoretische Physik

Diplom in Physik

Thema der Diplomarbeit: „Herleitung und Untersuchung der Feldund Bewegungsgleichungen von an das Gravitationsfeld gekoppelten Tensorfeld-Theorien“, Betreuer: Prof. Dr. Hubert Goenner Stipendiat des Graduiertenkollegs „Strömungsinstabilitäten und Turbulenz"

Anfertigung der vorliegenden Dissertation an der UniversitätsSternwarte Göttingen unter Anleitung von Prof. Dr. Klaus Beuermann 